City University of New York (CUNY) CUNY Academic Works

9-2016

\title{
Explicit Reciprocity Laws for Higher Local Fields
}

Jorge Florez

The Graduate Center, City University of New York

\section{How does access to this work benefit you? Let us know!}

More information about this work at: https://academicworks.cuny.edu/gc_etds/1469

Discover additional works at: https://academicworks.cuny.edu

This work is made publicly available by the City University of New York (CUNY).

Contact: AcademicWorks@cuny.edu 


\title{
Explicit Reciprocity Laws for Higher Local Fields
}

\author{
by \\ Jorge Florez
}

A dissertation submitted to the Graduate Faculty in Mathematics in partial fulfillment of the requirements for the degree of Doctor of Philosophy, The City University of New York. 
(C) 2016

Jorge Florez

All Rights Reserved 
This manuscript has been read and accepted for the Graduate Faculty in Mathematics in satisfaction of the dissertation requirements for the degree of Doctor of Philosophy.

Victor Kolyvagin

Date

Date
Chair of Examining Committee

Ara Basmajian

Executive Officer

Victor Kolyvagin

Carlos Moreno

Kenneth Kramer

Supervisory Committee

THE CITY UNIVERSITY OF NEW YORK 


\author{
Abstract \\ Explicit Reciprocity Laws for Higher \\ Local Fields \\ by \\ Jorge Florez
}

Advisor: Victor Kolyvagin

In this thesis we generalize to higher dimensional local fields the explicit reciprocity laws of Kolyvagin [14 for the Kummer pairing associated to a formal group. The formulas obtained describe the values of the pairing in terms of multidimensional $p$-adic differentiation, the logarithm of the formal group, the generalized trace and the norm on Milnor K-groups. 


\section{Acknowledgements}

I would like to start by thanking my adviser Victor Kolyvagin for suggesting the problem for this thesis and directions for future research. I am also thankful to him for sharing his mathematical ideas, and for the time he spent going over the manuscript providing valuable feedback to improve it. Moreover, I thank the committee members, Kenneth Kramer and Carlos Moreno for taking the time to read my paper and providing feedback.

I want to add an especial acknowledgment to Carlos Moreno for his friendship and for the guidance, support and motivation throughout my professional career.

I would also like to thank many people who were important in the culmination of this project. Among them Professor Jozef Dodziuk and Robert Landsman for their assistance and advise throughout these years. The mathematicians Jorge Basilio, Cihan Karabulut, Tian An Wong and Gabriel Zapata for their warm friendship. The afternoons we spent talking about mathematics had a great impact in my life; their constant encouragement made this reached goal possible.

Finally, I want to express my gratitude to my dear family for their love and 
support in this important phase of my life. 


\section{Contents}

0.1 Terminology and notation $\ldots \ldots \ldots \ldots$. . . . . . . . . . . 1

\begin{tabular}{lll}
\hline & Introduction & 5
\end{tabular}

1.1 Background . . . . . . . . . . . . . . . . . . 5

1.2 Description of the problem and results . . . . . . . . . . . . . . 8

2 Higher-dimensional local fields 15

2.1 2-dimensional local fields . . . . . . . . . . . . . . . . . . . 15

$2.2 d$-dimensional local fields . . . . . . . . . . . . . . . . . . . 21

2.3 Extensions of $\mathcal{K} \ldots \ldots \ldots \ldots$

2.4 Topology on $\mathcal{K} \ldots \ldots \ldots \ldots$

2.5 Topology on $\mathcal{K}^{*} \ldots \ldots \ldots \ldots \ldots \ldots$

\begin{tabular}{lll}
3 & Formal groups & 27 \\
\hline
\end{tabular}

3.1 The Weiertrass lemma . . . . . . . . . . . . . . . . . . . . . . 28

3.2 The group $F\left(\mu_{\mathcal{M}}\right) . \ldots \ldots \ldots \ldots$ 
3.3 The logarithm of the formal group . . . . . . . . . . . . . . . . . . 31

4 The Kummer Pairing 34

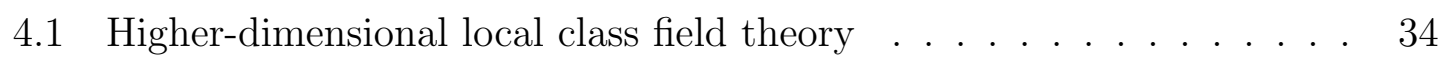

4.1 .1 Milnor-K-groups and norms . . . . . . . . . . . . . . 34

4.1 .2 The reciprocity map . . . . . . . . . . . . . . . . . . 39

4.2 The pairing $(,)_{\mathcal{L}, n} \ldots \ldots \ldots \ldots \ldots$. . . . . . . . . . . . 41

4.3 Norm Series . . . . . . . . . . . . . . . . . . . . . . . . . . . . . . 49

5 The maps $\psi$ and $\rho$

5.1 The generalized trace $\ldots \ldots \ldots \ldots$. . . . . . . . . . . . 52

5.2 The $\operatorname{map} \psi_{\mathcal{L}, n}^{i} \ldots \ldots \ldots \ldots$. . . . . . . . . . . . . . 64

5.3 The $\operatorname{map} \rho_{\mathcal{L}, n}^{i} \ldots \ldots \ldots \ldots \ldots \ldots \ldots$. . . . . . . . . . . . . . . 72

$5.4 \quad$ The $\operatorname{maps} D_{\mathcal{L}, n}^{i} \ldots \ldots \ldots \ldots \ldots \ldots$

6 Multidimensional derivations $\quad 82$

6.1 Derivations and the module of differentials . . . . . . . . . . . . . . . 82

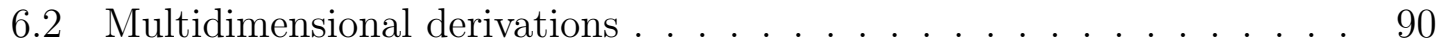

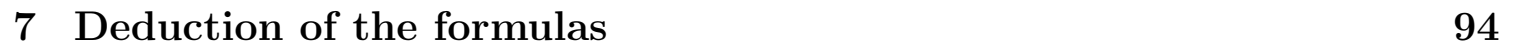

$7.1 \quad$ Description of the map $\psi_{\mathcal{M}, m}^{i}$ in terms of derivations . . . . . . . . . . 94

7.2 Invariants of the representation $\tau$. . . . . . . . . . . . . . . . . . . 99 
7.3 Main formulas . . . . . . . . . . . . . . . . . . . . . . . . . . . 109

8 Formulas for the Lubin-Tate formal groups 113

8.1 Computations of the invariants . . . . . . . . . . . . . 122

8.2 Explicit formulas for the Lubin-Tate formal groups . . . . . . . . . 128

\begin{tabular}{ll}
\hline Bibliography & 134
\end{tabular} 


\subsection{Terminology and notation}

For the convenience of the reader we will list some terminology and notation that we will use through out the paper. The letters $K, L, M$, will be used for one dimensional local fields and the corresponding script capital Latin symbols $\mathcal{K}, \mathcal{L}$ and $\mathcal{M}$ will denote higher local fields containing the corresponding local fields.

- $p$ denotes a fixed prime number.

- Let $K / \mathbb{Q}_{p}$ denote a local field with ring of integers $\mathcal{O}_{K}$.

- $F=F(X, Y) \in \mathcal{O}_{K}[[X, Y]]$ will denote a formal group of finite height $h$. Let $\operatorname{End}_{\mathcal{O}_{K}}(F)$ be the ring of endomorphisms of $F$ and $c: \operatorname{End}_{\mathcal{O}_{K}}(F) \rightarrow \mathcal{O}_{K}$ the embedding $g \mapsto c(g)=g^{\prime}(0)$.

- Let $S$ be a local field with ring of integers $C$ such that $C \subset c\left(\operatorname{End}_{\mathcal{O}_{K}}(F)\right)$. We will fix a uniformizer $\pi$ of $C$. Given $a \in C$, the endomorphism $a X+\cdots$ will be denoted by $[a]_{F}$. For convenience, $[\pi]_{F}$ will be denoted by $f$. Thus $f^{(n)}$, the $n$th fold of $f$, corresponds to $\left[\pi^{n}\right]_{F}$.

- Let $\kappa_{n} \simeq\left(C / \pi^{n} C\right)^{h}$ be the $\pi^{n}$ th torsion group of $F$ and let $\kappa=\lim _{\longleftarrow} \kappa_{n} \simeq C^{h}$. Let $\left\{e^{i}\right\}_{i=1}^{h}$ be a basis for $\kappa$ and $\left\{e_{n}^{i}\right\}_{i=1}^{h}$ be the corresponding reductions to the group $\kappa_{n}$. 
- Let $\alpha$ denote the ramification index of $S$ over $\mathbb{Q}_{p}$. We say that a pair $(n, t)$ is admissible if there exist an integer $k$ such that $t-1-n \geq \alpha k \geq n$. For example, the pair $(n, 2 n+\alpha+1)$ is admissible with $k=[(n+\alpha / \alpha)]$.

- If $R$ is a discrete valuation ring, the symbols $v_{R}, \mathcal{O}_{R}, \mu_{R}$ and $\pi_{R}$ will always denote the valuation, ring of integers, maximal ideal, and some fix uniformizer of $R$, respectively. Moreover, if the characteristic of the residue field is $p$, then we define $\mu_{R, 1}=\left\{x \in R: v_{R}(x)>v_{R}(p) /(p-1)\right\}$. If $R \supset K$, then $F\left(\mu_{R}\right)$ and $F\left(\mu_{R, 1}\right)$ will denote the group of $\mu_{R}$-points and $\mu_{R, 1}$-points of $F$, respectively.

- $\mathcal{L}$ is a $d$-dimensional complete field of characteristic 0, i.e., a field for which there are fields $\mathcal{L}_{d}=\mathcal{L}, \mathcal{L}_{d-1}, \ldots, \mathcal{L}_{0}$ such that $\mathcal{L}_{i+1}$ is a complete discrete valuation field with residue field $\mathcal{L}_{i}, 0 \leq i \leq d-1$, and $\mathcal{L}_{0}$ is a finite field of characteristic $p$.

- In what follows we assume that $\mathcal{L}$ is a field of mixed characteristic, i.e., $\operatorname{char}(\mathcal{L})=$ 0 and $\operatorname{char}\left(\mathcal{L}_{d-1}\right)=p$. Moreover, we also assume that $\mathcal{L} \supset K\left(\kappa_{n}\right)$.

- Let $t_{1}, \ldots, t_{d}$ be a fixed system of local parameters for $\mathcal{L}$, i.e, $t_{d}$ is a uniformizer for $\mathcal{L}=\mathcal{L}_{d}, t_{d-1}$ is a unit in $\mathcal{O}_{\mathcal{L}}$ but its residue in $\mathcal{L}_{d-1}$ is a uniformizer element of $\mathcal{L}_{d-1}$ and so on.

- Let $\mathrm{v}_{\mathcal{L}}=\left(v_{1}, \ldots, v_{d}\right): \mathcal{L}^{*} \rightarrow \mathbb{Z}^{d}$ be the valuation of rank $d$ corresponding to the system of parameters $t_{1}, \ldots, t_{d}$, i.e., $v_{d}=v_{\mathcal{L}_{d}}, v_{d-1}(\alpha)=v_{\mathcal{L}_{d-1}}\left(\alpha_{d-1}\right)$ 
where $\alpha_{d-1}$ is the residue of $\alpha / t_{d}^{-v_{d}(\alpha)}$ in $\mathcal{L}_{d-1}$, and so on. Here the group $\mathbb{Z}^{d}$ is ordered lexicographically as follows: $\bar{a}=\left(a_{1}, \ldots, a_{d}\right)<\left(b_{1}, \ldots, a_{d}\right)$ if $a_{l}<b_{l}$ and $a_{l+1}=b_{l+1}, \ldots, a_{d}=b_{d}$ for some $l \leq d$. We denote by $\mathbb{Z}_{+}^{d}=\left\{\left(a_{1}, \ldots, a_{d}\right) \in\right.$ $\left.\mathbb{Z}^{d}:\left(a_{1}, \ldots, a_{d}\right)>(0, \ldots, 0)\right\}$

- $O_{\mathcal{L}}=\left\{x \in \mathcal{L}: \bar{v}_{\mathcal{L}}(x) \geq \overline{0}\right\}$ is the valuation ring of rank $d$ of $\mathcal{L}$, with maximal ideal $M_{\mathcal{L}}=\left\{x \in \mathcal{L}: \bar{v}_{\mathcal{L}}(x)>\overline{0}\right\}$. Notice that $\mu_{\mathcal{L}} \subset M_{\mathcal{L}} \subset O_{\mathcal{L}} \subset \mathcal{O}_{\mathcal{L}}$.

- $\mathcal{V}_{\mathcal{L}}=1+M_{\mathcal{L}}$ is the group of principal units.

- The field $\mathcal{L}$ comes equipped with a special topology, the Parshin topology (cf.

- Observe that $\mathcal{L}^{*} \cong V_{\mathcal{L}} \times\left\langle t_{1}\right\rangle \times \cdots \times\left\langle t_{d}\right\rangle \times \mathcal{R}^{*}$, where $\mathcal{R}$ is a multiplicative closed system of representatives of the last residue field $\mathcal{L}_{0}$ and $\mathcal{R}^{*}=\mathcal{R} \backslash\{0\}$. Then we can endow $\mathcal{L}^{*} \cong V_{\mathcal{L}} \times\left\langle t_{1}\right\rangle \times \cdots \times\left\langle t_{d}\right\rangle \times \mathcal{R}^{*}$ with the product topology, where the group of principal units $\mathcal{V}_{\mathcal{L}}$ has the induced topology from $\mathcal{L}$, and $\left\langle t_{1}\right\rangle \times \cdots \times\left\langle t_{d}\right\rangle \times \mathcal{R}^{*}$ with the discrete topology

- $K_{d}(\mathcal{L})$ is the $d$ th Milnor $K$-group of the field $\mathcal{L}, d \geq 1$.

- Let $L$ be a complete discrete valuation field and define

$$
\mathcal{L}=L\{\{T\}\}=\left\{\sum_{-\infty}^{\infty} a_{i} T^{i}: a_{i} \in L, \inf v_{L}\left(a_{i}\right)>-\infty, \lim _{i \rightarrow-\infty} v_{L}\left(a_{i}\right)=+\infty\right\}
$$


Let $v_{\mathcal{L}}\left(\sum a_{i} T^{i}\right)=\min _{i \in \mathbb{Z}} v_{L}\left(a_{i}\right)$, so $\mathcal{O}_{\mathcal{L}}=\mathcal{O}_{L}\{\{T\}\}$ and $\mu_{\mathcal{L}}=\mu_{L}\{\{T\}\}$. Observe that $\mathcal{O}_{\mathcal{L}} / \mu_{\mathcal{L}}=k_{L}((\bar{T}))$, where $k_{L}$ is the residue field of $L$.

- We define

$$
L\left\{\left\{T_{1}\right\}\right\} \cdots\left\{\left\{T_{d-1}\right\}\right\}
$$

inductively by $E_{d-1}\{\{T\}\}$ where $E_{d-1}=L\left\{\left\{T_{1}\right\}\right\} \cdots\left\{\left\{T_{d-2}\right\}\right\}$.

- $D(L / K)$ will denote the different of the the extension of local fields $L / K$. 


\section{Chapter 1}

\section{Introduction}

\section{$1.1 \quad$ Background}

The history for finding explicit formulations of local class field theory dates back to Kummer [15] in 1858 when he tried to describe the Hilbert symbol, associated to the cyclotomic field $\mathbb{Q}_{p}\left(\zeta_{p}\right)$, in terms of logarithmic derivatives. Seventy years later, when class field theory was acquiring its final shape, Artin and Hasse [1] provided an explicit formula for the Hilbert symbol associated to $\mathbb{Q}_{p}\left(\zeta_{p^{m}}\right) ; p$ being an odd prime.

The formulas of Artin-Hasse would find an application in the work of Iwasawa [10] when he studied the units of the cyclotomic field $\mathbb{Q}\left(\zeta_{p^{m}}\right)$. Iwasawa extended the formulas of Artin-Hasse to describe more values of the Hilbert symbol (cf. [9]), in terms of $p$-adic logarithmic derivatives. This would be the first manifestation of a deep connection, via explicit reciprocity laws, between arithmetical objects and values of zeta functions. The work of Iwasawa inspired Coates and Wiles [3] to do fundamental work on the Birch and Swinnerton Dyer conjecture. One of the main 
tools in their paper [27] was to extend Iwasawa's reciprocity laws to Kummer pairings associated to Lubin-Tate formal groups, which are analogues of the Hilbert symbol for formal groups.

Soon after, Kolyvagin [14] extended the formulas of Wiles from Lubin-Tate formal groups to arbitrary formal groups (of finite height) and described the Kummer pairing in terms of $p$-adic derivations. Moreover, Kolyvagin also showed that the pairing is completely characterized by its values at the torsion points of the formal group.

Parallel to this, the theory of higher local fields was developing and along with it was the class field theory for such fields. Different formulations for a such theory were proposed by many people but it would be Kato's [11] (see also [22]), who by means of Milnor K-groups, developed the most commonly used theory. Kato's formulation shows that the finite abelian extensions of a $d$-dimensional higher local field correspond to norm subgroups of its Milnor $d$ th K-group. This correspondence was established via a reciprocity map between the $d$ th Milnor $K$-group and the galois group of the maximal abelian extension of the higher local field. I. Fesenko 4] nicely contributed to the class field theory for higher local fields also.

After Kato developed this class field theory, the program to make this theory more explicit began. Some of the higher dimensional explicit reciprocity laws that can be found in the literature are those of Kurihara [16], Vostokov [25], [26], Zinoviev [28] and Kato [12]. These explicit laws describe the analogue of Hilbert symbol for 
higher local fields, in terms of higher dimensional $p$-adic logarithmic differentiation. Using his explicit reciprocity laws, Kato made fundamental progress on Iwasawa main conjecture for modular forms. Kato's explicit laws provide a link between the Euler system of Beilinson elements and some special values of the $L$-function attached to a modular form. This allowed Kato [13] to prove a divisibility statement related to the Iwasawa main conjecture for modular forms.

In this thesis, we will derive explicit reciprocity laws for the higher dimensional analogue of the Kummer pairing associated to a formal group $F$, or generalized Kummer pairing. The construction of these laws involve higher dimensional $p$-adic derivations, the logarithm of the formal group $F$, the generalized trace and the norm of Milnor $K$-groups. These formulas constitute a generalization, to higher local fields, of the explicit reciprocity laws of Kolyvagin [14]. In particular, the formulas apply to the generalized Hilbert symbol, like those of Kurihara [16] and Zinoviev [28]. It is still a work in progress to determine if the formulas of these two authors can be derived from the explicit reciprocity laws constructed in this thesis.

Finally, it is worth mentioning that the techniques that are used to obtain the explicit reciprocity laws are inspired by the work of Kolyvagin in [14]. In particular, this allows for a more classical and conceptual approach to the higher dimensional reciprocity laws. 


\subsection{Description of the problem and results}

Let $K / \mathbb{Q}_{p}$ be a local field and $F$ a one-dimensional formal group of finite height with coefficients in the ring of integers of $K$. Let $\operatorname{End}_{\mathcal{O}_{K}}(F)$ be the ring of endomorphisms of $F$ and $c: \operatorname{End}_{\mathcal{O}_{K}}(F) \rightarrow \mathcal{O}_{K}$ the embedding $f \mapsto c(f)=f^{\prime}(0)$. Let $S / \mathbb{Q}_{p}$ be a local field with ring of integers $C$ such that $C \subset c\left(\operatorname{End}_{\mathcal{O}_{K}}(F)\right) \subset \mathcal{O}_{K}$. Let us fix a uniformizer $\pi$ for $S$ and denote by $f=[\pi]_{F}$ the endomorpshism such that $c(f)=\pi$.

Let $h$ be the height of the endomorphism $[\pi]_{F}$ with respect to $C$, i.e, $[\pi]_{F}(X)=$

$g\left(X^{q^{h}}\right)(\bmod \pi C)$ where $g^{\prime}(0) \neq 0(\bmod \pi C)$ and $q=\left|k_{S}\right|$. Denote by $f^{(n)}=\left[\pi^{n}\right]_{F}$ the $n$-fold of $f$. Let $\kappa_{n}$ be the $\pi^{n}$ th torsion group, i.e., the set of those $v \in \bar{K}$ such that $f^{(n)}(v)=0$. Let $\left\{e_{n}^{i}\right\}_{i=1}^{h}$ be a $C / \pi^{n} C$-basis for the group $\kappa_{n} \simeq\left(C / \pi^{n} C\right)^{h}$.

Let $L \supset K$ be a local field containing $\kappa_{n}$. Let $\mathcal{O}_{L}$ be the ring of integers of $L, \mu_{L}$ its maximal ideal, $v_{L}$ its valuation and $\pi_{L}$ be a uniformizer for $L$. Define

$$
\mathcal{L}=L\left\{\left\{T_{1}\right\}\right\} \cdots\left\{\left\{T_{d-1}\right\}\right\}
$$

inductively. The ring on integers and maximal ideal are, respectively,

$$
\mathcal{O}_{\mathcal{L}}=\mathcal{O}_{L}\left\{\left\{T_{1}\right\}\right\} \cdots\left\{\left\{T_{d-1}\right\}\right\} \quad \text { and } \quad \mu_{\mathcal{L}}=\mu_{L}\left\{\left\{T_{1}\right\}\right\} \cdots\left\{\left\{T_{d-1}\right\}\right\}
$$

We define the canonical pairing (cf. $\S 4.1$ )

$$
(,)_{\mathcal{L}, n}: K_{d}(\mathcal{L}) \times F\left(\mu_{\mathcal{L}}\right) \rightarrow \kappa_{n}
$$


by

$$
(\alpha, x) \mapsto \Upsilon_{\mathcal{L}}(\alpha)(z)-{ }_{F} z
$$

where $K_{d}(\mathcal{L})$ is the $d$ th Milnor $K$-group of $\mathcal{L}, \Upsilon_{\mathcal{L}}: K_{d}(\mathcal{L}) \rightarrow G_{\mathcal{L}}^{a b}$ is the Kato's reciprocity map for $\mathcal{L}, f^{(n)}(z)=x$ and $-_{F}$ is the subtraction in the formal group $F$, cf. $\S 4$.1. Denote by $(,)_{\mathcal{L}, n}^{i}$ the $i$ th coordinate of $(,)_{\mathcal{L}, n}$ with respect to the basis $\left\{e_{n}^{i}\right\}$.

In this paper we will use the technique of Kolyvagin in 14 to derive explicit formulas for the symbol $(,)_{\mathcal{L}, n}^{i}$ in terms of the generalized trace, the norm of Milnor $K$-groups, $d$-dimensional derivations and the logarithm of the formal group.

More specifically, let $E$ be a complete discrete valuation field. We define a map

$$
c_{E\{\{T\}\} / E}: E\{\{T\}\} \rightarrow E
$$

by $c_{E\{\{T\}\} / E}\left(\sum_{i \in \mathbb{Z}} a_{i} T^{i}\right)=a_{0}$. Then we can define $c_{\mathcal{L} / L}$ by the composition

$$
c_{L\left\{\left\{T_{1}\right\}\right\} / L} \circ \cdots \circ c_{\mathcal{L} / L\left\{\left\{T_{1}\right\}\right\} \ldots\left\{\left\{T_{d-2}\right\}\right.}
$$

We define the generalized trace by $\mathbb{T}_{\mathcal{L} / S}=\operatorname{Tr}_{L / S} \circ c_{\mathcal{L} / L}$.

The main result in this paper is the following (cf. Theorem 7.3.1). Let $M=L\left(\kappa_{t}\right)$, $t \geq m \geq n$, for $t$ large enough with respect to $m$ and put $\mathcal{M}=M\left\{\left\{T_{1}\right\}\right\} \cdots\left\{\left\{T_{d-1}\right\}\right\}$, then

$$
\left(N_{\mathcal{M} / \mathcal{L}}\left\{a_{1}, \ldots, a_{d}\right\}, x\right)_{\mathcal{L}, n}^{i}=\mathbb{T}_{\mathcal{M} / S}\left(\frac{D_{\mathcal{M}, m}^{i}\left(a_{1}, \ldots, a_{d}\right)}{a_{1} \cdots a_{d}} l_{F}(x)\right)
$$

for all $a_{1}, \ldots, a_{d} \in \mathcal{M}^{*}$ (see 7.1 where cases when $a_{i}$ are units or not are considered) 
and all $x \in F\left(\mu_{\mathcal{L}}\right)$. Here $l_{F}$ is the formal logarithm, $N_{\mathcal{M} / \mathcal{L}}$ is the norm on Milnor $K$-groups and $D_{\mathcal{M}, m}^{i}$ is a $d$-dimensional derivation of $\mathcal{O}_{\mathcal{L}}$ over $\mathcal{O}_{K}$, i.e.,

$$
D_{\mathcal{M}, m}^{i}: \mathcal{O}_{\mathcal{M}}^{d} \rightarrow W
$$

is a map, with $W$ an $\mathcal{O}_{\mathcal{M}}$-module, satisfying the properties in definition 6.2.1, that is, for all $a_{1}, \ldots, a_{d}$ and $a_{1}^{\prime}, \ldots, a_{d}^{\prime}$ in $\mathcal{O}_{\mathcal{M}}$ we have:

1. Leibniz rule:

$$
D\left(a_{1}, \ldots, a_{i} a_{i}^{\prime}, \ldots, a_{d}\right)=a_{i}^{\prime} D\left(a_{1}, \ldots, a_{i}, \ldots, a_{d}\right)+a_{i} D\left(a_{1}, \ldots, a_{i}^{\prime}, \ldots, a_{d}\right),
$$

for any $1 \leq i \leq d$.

2. Linearity:

$$
D\left(a_{1}, \ldots, a_{i}+a_{i}^{\prime}, \ldots, a_{d}\right)=D\left(a_{1}, \ldots, a_{i}, \ldots, a_{d}\right)+D\left(a_{1}, \ldots, a_{i}^{\prime}, \ldots, a_{d}\right),
$$

for any $1 \leq i \leq d$.

3. Alternate: $D\left(a_{1}, \ldots, a_{i}, \ldots, a_{j}, \ldots, a_{d}\right)=0$, if $a_{i}=a_{j}$ for $i \neq j$.

4. $D\left(a_{1}, \ldots, a_{d}\right)=0$ if some $a_{i} \in \mathcal{O}_{K}$.

Notice that $D$ is a 1-dimensional derivation in each component and moreover, it can be parametrized by 1-dimensional derivations. Indeed, just as one dimensional

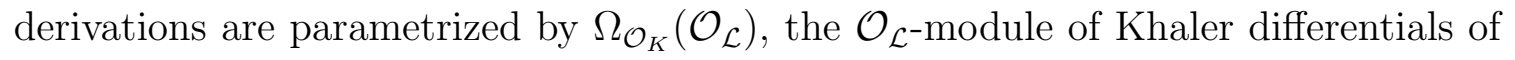


$\mathcal{O}_{\mathcal{L}}$ over $\mathcal{O}_{K}$, then it will also be shown in $\S 6.2$ Proposition 6.2.1 that all such $d$ dimensional derivations are parametrized by the $\mathcal{O}_{\mathcal{L}}$-module $\bigwedge^{d} \Omega_{\mathcal{O}_{K}}\left(\mathcal{O}_{\mathcal{L}}\right)$, i.e., the

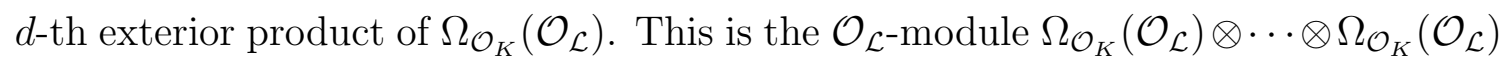

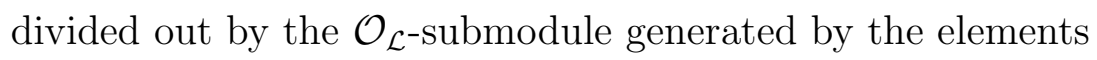

$$
x_{1} \otimes \cdots \otimes x_{d}
$$

where $x_{i}=x_{j}$ for some $i \neq j$, where $x_{1}, \ldots, x_{d} \in \Omega_{\mathcal{O}_{K}}\left(\mathcal{O}_{\mathcal{L}}\right)$.

Moreover, notice that from properties (2), (3) and (4) it easily follows that $D$ satisfies the relations

$$
D\left(a_{1}, \ldots, a_{i}, \ldots, a_{j} \ldots, a_{d}\right)=0
$$

if $a_{i}+a_{j}=1$, for $i \neq j$, which are called the Steinberg relations. Therefore, following Kolyvagin [14], we define the map

$$
\psi:\left(\mathcal{O}_{\mathcal{M}}^{\times}\right)^{d} \rightarrow W
$$

by

$$
\left\{a_{1}, \ldots, a_{d}\right\} \rightarrow \frac{D_{\mathcal{M}, m}^{i}\left(a_{1}, \ldots, a_{d}\right)}{a_{1} \ldots a_{d}}
$$

This map can be extended to all of $K_{d}\left(\mathcal{M}^{*}\right)$ by equation 7.1 from Section 7.1 .

As in the work of Kolyvagin [14, these derivations will be normalized in terms of the invariants (7.7) attached to the Galois representation on the Tate-module (7.5).

More specifically, we will build the $d$-dimensional derivation $D_{\mathcal{M}, m}^{i}$ out of the 
condition

$$
D_{\mathcal{M}, m}^{i}\left(T_{1}, \ldots, T_{d-1}, e_{t}^{j}\right)=-\frac{T_{1} \cdots T_{d-1} c_{i, j}}{l^{\prime}\left(e_{t}^{j}\right)},
$$

cf. Proposition 7.2.3, where $c_{i, j}$ is defined in equation (7.7).

The deduction of the formula starts by proving that the pairing $(,)_{\mathcal{L}, n}$ is sequentially continuous in the second argument with respect to the Parshin topology, i.e., if $\alpha \in K_{d}(\mathcal{L})$ and $\left\{x_{k}\right\}$ is a sequence in $F\left(\mu_{\mathcal{L}}\right)$ such that $x_{k} \rightarrow 0$ then $\left(\alpha, x_{k}\right)_{\mathcal{L}, n} \rightarrow 0$, cf. Proposition 4.2.2, which is vital for the rest of the proof and was inspired by the proof of Proposition 4.1 in [14]. From this, we can prove the existence of the so called Iwasawa maps, $\psi_{\mathcal{L}, n}^{i}: K_{d}(\mathcal{L}) \rightarrow R_{\mathcal{L}, 1} / \pi^{n} R_{\mathcal{L}, 1}$ (cf. Proposition 5.2.1), such that

$$
(\alpha, x)_{\mathcal{L}, n}^{i}=\mathbb{T}_{\mathcal{L} / S}\left(\psi_{\mathcal{L}, n}^{i}(\alpha) l_{F}(x)\right) \quad \forall x \in F\left(\mu_{\mathcal{L}, 1}\right)
$$

where $F\left(\mu_{\mathcal{L}, 1}\right)$ is the set $\left\{x \in \mathcal{L}: v_{\mathcal{L}}(x) \geq\left[v_{\mathcal{L}}(p) /(p-1)\right]+1\right\}$ considered with the operation defined by $F$. We then define the maps $D_{\mathcal{L}, n}^{i}$ out of $\psi_{\mathcal{L}, n}^{i}$ as in equation 5.17. The main goal is to prove that under certain conditions the map $D_{\mathcal{M}, m}^{i}$ is a $d$-dimensional derivation over $\mathcal{O}_{K}$, cf. Proposition 7.1.1. A vital role in this paper is given by the norm series relations

$$
\left(\left\{r(x), a_{2}, \ldots, a_{d}\right\}, x\right)_{\mathcal{L}, n}=0, \quad \forall x \in F\left(\mu_{\mathcal{L}}\right), \forall a_{2}, \ldots, a_{d} \in \mathcal{L}^{*}
$$

cf. Proposition 4.3.1. These series allow us to obtain the representation (5.8) in Proposition 5.2 .3 which together with the continuity of the pairing we obtain the 
identity (5.18) from Proposition 5.4.2. But Proposition 5.4.4 tells us that every element in $\mu_{\mathcal{L}}$ has a representation of the form $\eta\left(T_{1}, \ldots, T_{d-1}, \pi_{L}\right)$, where $\eta\left(X_{1}, \ldots, X_{d}\right)$ is a power series in the variables $X_{1}, \ldots, X_{d}$ with coefficients in $\mathcal{O}_{L}$, given by equation (5.4.5).

Then, we will conclude from Proposition 5.4.5 and corollary 5.4.4 that

$$
D_{\mathcal{L}, m}^{i}\left(\alpha_{1}, \ldots, \alpha_{d}\right)=\left.\operatorname{det}\left[\frac{\partial \eta_{i}}{\partial X_{j}}\right]_{i, j}\right|_{\substack{X_{k}=T_{k}, k=1, \ldots, d}} D_{\mathcal{L}, n}^{i}\left(T_{1}, \ldots, \pi_{L}\right)
$$

where $\alpha_{i}=\eta_{i}\left(T_{1}, \ldots, \pi_{L}\right), i=1, \ldots, d$ and $T_{d}=\pi_{L}$. This last equation tells us that $D_{\mathcal{L}, n}^{i}$ behaves like a $d$-dimensional derivation when restricted to $\mu_{\mathcal{L}}$.

This will be used in the proof of Proposition 7.1.1 to conclude that $D_{\mathcal{M}, m}^{i}$, for $\mathcal{M}=$ $M\left\{\left\{T_{1}\right\}\right\} \cdots\left\{\left\{T_{d-1}\right\}\right\}$ and $M$ a finite extension of $L$ containing $\kappa_{t}$ for large enough $t$ with respect to $m$, is a $d$-dimensional derivation when restricted to certain quotient of $\mathcal{M}$ as an $\mathcal{O}_{\mathcal{M}}$-module, namely $R_{\mathcal{M}, 1} /\left(\pi^{m} / \pi_{M}\right) R_{\mathcal{M}, 1}$. Moreover, Proposition 7.2 .3 gives us a way of constructing explicitly this derivation out of the condition

$$
D_{\mathcal{M}, m}^{i}\left(T_{1}, \ldots, T_{d-1}, e_{t}^{j}\right)=-T_{1} \cdots T_{d-1} c_{i, j} / l^{\prime}\left(e_{t}^{j}\right)
$$

We can express back the Iwasawa map $\psi_{\mathcal{M}, m}^{i}: K_{d}(\mathcal{M}) \rightarrow R_{\mathcal{M}, m} / \pi^{m} R_{\mathcal{M}, m}$ in terms of the derivation $D_{\mathcal{M}, m}^{i}$ as

$$
\psi_{\mathcal{M}, m}^{i}\left(\left\{a_{1}, \ldots, a_{d}\right\}\right)=\frac{D_{\mathcal{M}, m}^{i}\left(a_{1}, \ldots, a_{d}\right)}{a_{1} \cdots a_{d}}
$$


cf. 7.1. Finally, the return to the field $\mathcal{L}$ will be guaranteed by Proposition 7.1.2. The final formulas are contained in Theorem 7.3.1.

In chapter 8 we specialize to the case of a Lubin-tate formal group and refine Theorem 7.3.1 for this case. In particular, we give an explicit computation of the invariants $c_{i, j}$, namely

$$
c_{i, j}=-\frac{1}{\pi^{t}} .
$$




\section{Chapter 2}

\section{Higher-dimensional local fields}

\section{$2.1 \quad 2$-dimensional local fields}

We will start defining 2-dimensional local fields since most of the proofs will be reduced to this case. In the next sections all the concepts will be generalized to higher dimensions.

Definition 2.1.1. We say that $\mathcal{K}$ is a 2-dimensional local field if there are fields $\mathcal{K}_{2}=\mathcal{K}, \mathcal{K}_{1}$, and $\mathcal{K}_{0}$ such that $\mathcal{K}_{i+1}$ is a complete discrete valuation ring with residue field $\mathcal{K}_{i}, 0 \leq i \leq 1$, and $\mathcal{K}_{0}$ is a finite field of characteristic $p$.

We have the following examples of 2-dimensional local fields:

1. $\mathbb{F}_{q}\left(\left(T_{1}\right)\right)\left(\left(T_{2}\right)\right)$, where $q$ is a power of the prime $p$. In this case $\mathcal{K}_{2}=\mathbb{F}_{q}\left(\left(T_{1}\right)\right)\left(\left(T_{2}\right)\right)$, $\mathcal{K}_{1}=\mathbb{F}_{q}\left(\left(T_{1}\right)\right)$ and $K_{0}=\mathbb{F}_{q}$. Recall that if $k$ is a field then $k((T))$ is the fraction field of the ring $k[[T]]$.

2. $E((T))$, where $E$ is a finite extension of $\mathbb{Q}_{p}$. Here $\mathcal{K}_{1}=E$ and $\mathcal{K}_{0}=k_{E}$ 
3. For a complete discrete valuation field $E$ we define

$$
\mathcal{K}=E\{\{T\}\}=\left\{\sum_{-\infty}^{\infty} a_{i} T^{i}: a_{i} \in E, \inf v_{E}\left(a_{i}\right)>-\infty, \lim _{i \rightarrow-\infty} v_{E}\left(a_{i}\right)=+\infty\right\}
$$

Let $v_{\mathcal{K}}\left(\sum a_{i} T^{i}\right)=\min v_{E}\left(a_{i}\right)$. Then $\mathcal{K}$ is a complete discrete valuation field with residue field $k_{F}((t))$ and ring of integers

$$
\mathcal{O}_{E\{\{T\}\}}=\left\{\sum_{-\infty}^{\infty} a_{i} T^{i} \in F\{\{T\}\}: a_{i} \in \mathcal{O}_{F}, \forall i \in \mathbb{Z}\right\}
$$

Thus, if $E$ is a local field then $E\{\{T\}\}$ is a 2-dimensional local field with $\mathcal{K}_{1}=k_{E}((t))$ and $\mathcal{K}_{0}=k_{E}$. These fields are called standard 2-dimensional local fields (cf. [7] §1.1).

We are interested only in the case where $\mathcal{K}$ is of mixed characteristic, i.e., $\operatorname{char}\left(\mathcal{K}_{1}\right)=$ $p$. The following Theorem classifies such fields.

Theorem 2.1.1 (Classification Theorem). Let $\mathcal{K}$ be a 2-dimensional local field of mixed characteristic. Then $\mathcal{K}$ is a finite extension of a 2-dimensional standard field $E\left\{\left\{T_{1}\right\}\right\}$, where $E$ is a finite extension of $\mathbb{Q}_{p}$, and there is a finite extension of $\mathcal{K}$ which is a standard 2-dimensional local field.

Proof. cf. [7] §1.1 Classification Theorem.

We define the ring of integers of $\mathcal{K}$ to be the set

$$
O_{\mathcal{K}}=\left\{x \in \mathcal{O}_{\mathcal{K}}: \bar{x} \in \mathcal{O}_{K_{1}}\right\}
$$


and the maximal ideal

$$
M_{\mathcal{K}}=\left\{x \in \mathcal{O}_{\mathcal{K}}: \bar{x} \in \mu_{K_{1}}\right\}
$$

where $\bar{x}$ denotes the reduction of $x$ in $\mathcal{K}_{1}$. Notice that

$$
\mu_{\mathcal{K}} \subset M_{\mathcal{K}} \subset O_{\mathcal{K}} \subset \mathcal{O}_{\mathcal{K}}
$$

In the examples above we can compute these two sets explicitly:

1. If $\mathcal{K}=\mathbb{F}_{q}\left(\left(T_{1}\right)\right)\left(\left(T_{2}\right)\right): O_{\mathcal{K}}=\mathbb{F}_{q}\left[\left[T_{1}\right]\right]+T_{2} \mathbb{F}_{q}\left(\left(T_{1}\right)\right)\left[\left[T_{2}\right]\right]$ and $M_{\mathcal{K}}=T_{1} \mathbb{F}_{q}\left[\left[T_{1}\right]\right]+$ $T_{2} \mathbb{F}_{q}\left(\left(T_{1}\right)\right)\left[\left[T_{2}\right]\right]$

2. If $\mathcal{K}=E((T)): O_{\mathcal{K}}=\mathcal{O}_{E}+T E[[T]]$ and $M_{\mathcal{K}}=\pi_{E} \mathcal{O}_{E}+T E[[T]]$.

3. If $\mathcal{K}=E\{\{T\}\}: O_{\mathcal{K}}=\left\{\sum_{-\infty}^{\infty} a_{i} T^{i} \in \mathcal{K}: a_{i} \in \mathcal{O}_{E}, \forall i \geq 0\right.$ and $\left.a_{i} \in \mu_{E} \forall i<0\right\}$ and $M_{\mathcal{K}}=\left\{\sum_{-\infty}^{\infty} a_{i} T^{i} \in F\{\{T\}\}: a_{i} \in \mathcal{O}_{E}, \forall i>0\right.$ and $\left.a_{i} \in \mu_{E} \forall i \leq 0\right\}$

In particular, if $\mathcal{K}=\mathbb{Q}_{p}\{\{T\}\}$ we have

$$
O_{\mathcal{K}}=\left\{\sum_{-\infty}^{\infty} a_{i} T^{i} \in \mathcal{K}: a_{i} \in \mathbb{Z}_{p}, \forall i \geq 0 \text { and } a_{i} \in p \mathbb{Z}_{p} \forall i<0\right\}
$$

and

$$
M_{\mathcal{K}}=\left\{\sum_{-\infty}^{\infty} a_{i} T^{i} \in \mathcal{K}: a_{i} \in \mathbb{Z}_{p}, \forall i>0 \text { and } a_{i} \in p \mathbb{Z}_{p} \forall i \leq 0\right\} .
$$

Topology on $\mathcal{K}$ : The topology on $\mathcal{K}=E\{\{T\}\}$ is called the Parshin topology 
or Higher-dimensional topology (cf. [7] $\S 1.3$ ). A basis of neighborhoods of 0 for this topology is given by the following: Let $\left\{U_{i}\right\}_{i \in \mathbb{Z}}$ be a sequence of neighborhoods of 0 in the local field $E$, i.e., $U_{i}=\pi_{E}^{n_{i}} \mathcal{O}_{E}$, where $n_{i} \in \mathbb{Z}$, such that

1. There exist a $c \in \mathbb{Z}$ such that $\pi_{E}^{c} \mathcal{O}_{E} \subset U_{i}$ for all $i \in \mathbb{Z}$, i.e., $\cap U_{i} \supset \pi_{E}^{c} \mathcal{O}_{E}$. This says that the neighborhoods $U_{i}$ 's cannot be arbitrarily small.

2. For every $l \in \mathbb{Z}$ we have $\pi_{E}^{l} \mathcal{O}_{E} \subset U_{i}$ for sufficiently large $i$. This implies that the $U_{i}$ 's get bigger as $i \rightarrow \infty$.

We put

$$
\mathcal{U}_{\left\{U_{i}\right\}}=\left\{\sum_{-\infty}^{\infty} a_{i} T^{i} \in \mathcal{K}: a_{i} \in U_{i}\right\}
$$

These sets $\mathcal{U}_{\left\{U_{i}\right\}}$ form a base of neighborhoods of 0 in $\mathcal{K}$.

Remark 2.1.1. Observe that in this topology an element $x=\sum_{-\infty}^{\infty} a_{i} T^{i} \in \mathcal{K}=$ $E\{\{T\}\}$ converges as an infinite sum since both tails $\sum_{i>N} a_{i} T^{i}$ and $\sum_{i<-N} a_{i} T^{i}$ approach to 0 as $N \rightarrow \infty$. To see this take a basis element for zero, $\mathcal{U}_{\left\{U_{i}\right\}}$, and let $c$ be given by condition 1 above, and for $l=v_{K}(x)=\inf v_{E}\left(a_{i}\right)$ let $N$ be large enough to satisfy condition 2 above.

Since by definition $v_{E}\left(a_{i}\right) \rightarrow \infty$ as $i \rightarrow-\infty$ we can find an $N$ such that $v_{E}\left(a_{i}\right)>c$ for $i<-N$. Then $a_{i} \in \pi_{E}^{c} \mathcal{O}_{E} \subset U_{i}$ for $i<-N$, i.e., $\sum_{i<-N} a_{i} T^{i} \in \mathcal{U}_{\left\{U_{i}\right\}}$. 
Now $v_{E}\left(a_{i}\right) \geq l$ for all $i$, then $a_{i} \in \pi_{E}^{l} \mathcal{O}_{E} \subset U_{i}$ for $i>N$. Again, this implies $\sum_{i>N} a_{i} T^{i} \in \mathcal{U}_{\left\{U_{i}\right\}}$

Remark 2.1.2. Observe also that a sequence $x_{n}=\sum_{-\infty}^{\infty} a_{i}^{(n)} T^{i}$ converging to zero in this topology does not necessarily satisfy that $v_{\mathcal{K}}\left(x_{n}\right) \rightarrow \infty$, i.e., the coefficients $a_{i}^{(n)}$ do not necessarily converge uniformly on $i$ to zero. For instance, the sequence $x_{n}=T^{n}$ converges to zero in this topology and $v_{\mathcal{K}}\left(x_{n}\right)=0$ for all $n$. But the following Proposition is true:

Proposition 2.1.1. The sequence $x_{n}=\sum_{-\infty}^{\infty} a_{i}^{(n)} T^{i}$ converges to zero if and only if the following two conditions are satisfied

(i) $\inf v_{\mathcal{K}}\left(x_{n}\right)>-\infty$;

(ii) for a given $m \in \mathbb{Z}_{+}$the sequence $a_{i}^{(n)} \stackrel{n \rightarrow \infty}{\rightarrow} 0$ uniformly for every $i \leq m$.

That is, the coefficients are uniformly bounded and converge to zero uniformly for $i \leq m$, for every $m$.

Proof. $\rightarrow$ ) Suppose the sequence $x_{n}$ converges to zero. For a fix $m \in \mathbb{Z}$ and given $M>0$ define

$$
U_{i}^{M}= \begin{cases}\pi_{E}^{M} \mathcal{O}_{E}, & i \leq m \\ \pi_{E}^{-i} \mathcal{O}_{E}, & i>m\end{cases}
$$

Then $\mathcal{V}_{M}=\mathcal{V}_{U_{i}^{M}}$ is a basis element for zero, so there exists an $N$ such that $x_{n} \in \mathcal{V}_{M}$ for $n>N$. That is $v_{E}\left(a_{i}^{(n)}\right) \geq M$ for $n>N$ and all $i \leq m$, then $a_{i}^{(n)} \stackrel{n \rightarrow \infty}{\rightarrow} 0$ uniformly on every $i \leq m$. 
Let us show now that $\inf v_{K}\left(x_{n}\right)>-\infty$. For $i>1$ define $m_{i}=\inf _{n} v_{E}\left(a_{i}^{(n)}\right)$ and $M_{i}=\min _{1 \leq k \leq i}\left\{m_{k}\right\}$. Then $M_{i} \geq M_{i+1}$ and moreover, if inf $v_{K}\left(x_{n}\right)=-\infty$, then $M_{i} \stackrel{i \rightarrow \infty}{\rightarrow}-\infty$ which follows from the just proven condition $(i i)$. Consider

$$
U_{i}= \begin{cases}\pi_{E} \mathcal{O}_{E}, & i \leq 0 \\ \pi_{E}^{M_{i}+1} \mathcal{O}_{E}, & i>0\end{cases}
$$

Then $\mathcal{U}_{\left\{U_{i}\right\}}$ is a base element for 0 . Again, since we are assuming $\inf v_{K}\left(x_{n}\right)=-\infty$, for certain large enough $i$ we have the strict inequality $M_{i-1}>M_{i}$, i.e., $M_{i}=m_{i}$ and so we can find a subsequence $x_{n_{i}}=\sum_{j} a_{j}^{\left(n_{i}\right)} T^{j}$ such that $v_{K}\left(a_{i}^{\left(n_{i}\right)}\right)=M_{i}$, thus $v_{K}\left(x_{n_{i}}\right) \leq v_{E}\left(a_{i}^{\left(n_{i}\right)}\right)=M_{i}$. Then $a_{i}^{\left(n_{i}\right)} \notin U_{i}$, which implies $x_{n_{i}} \notin \mathcal{U}_{\left\{U_{i}\right\}}$. Finally, since $n_{i} \rightarrow \infty$ as $i \rightarrow \infty$ this contradicts the convergence of $x_{n}$ to zero.

$\leftarrow)$ Let $x_{n}$ be a sequence in $K$ satisfying $(i)$ and $(i i)$. Let $\mathcal{U}_{\left\{U_{i}\right\}}$ is a base element for 0. Then there exist a $c \in \mathbb{Z}$ such that $\pi_{E}^{c} \mathcal{O}_{E} \subset U_{i}$ for all $i \in \mathbb{Z}$. Also for $l=\inf v_{K} x_{n}$ there exist an $i_{0}$ such that $\pi_{E}^{l} \mathcal{O}_{E} \subset U_{i}$ for all $i>i_{0}$.

By condition (ii) applied to $m=i_{0}$ there exist an $N$ such that $v_{E}\left(a_{i}^{(n)}\right)>c$ for all $n \geq N$ and all $i \leq i_{0}$. Then $a_{i}^{(n)} \in \pi_{E}^{c} \mathcal{O}_{E} \subset U_{i}$ for all $n \geq N$ and all $i \leq i_{0}$. On the other hand, $v_{E}\left(a_{i}^{(n)}\right) \geq \inf v_{K}\left(x_{n}\right)=l$, so $a_{i}^{(n)} \in \pi_{E}^{l} \mathcal{O}_{E} \subset U_{i}$ for all $i>i_{0}$. Summarizing we have that $a_{i}^{(n)} \in U_{i}$ for all $n>N$ and all $i \in \mathbb{Z}$. Therefore $x_{n} \in \mathcal{U}_{\left\{U_{i}\right\}}$ and so it converges to zero in the Parshin topology. 


\section{Topology on $\mathcal{K}^{*}$ :}

Let $\mathcal{R} \subset \mathcal{K}=\mathcal{K}_{2}$ be a set of representatives of the last residue field $\mathcal{K}_{0}$. Let $t_{1}$ and $t_{2}$ be a fixed system of local parameters for $\mathcal{K}$, i.e, $t_{2}$ is a uniformizer for $\mathcal{K}$ and $t_{1}$ is a unit in $\mathcal{O}_{\mathcal{K}}$ but its residue in $\mathcal{K}_{1}$ is a uniformizer element of $\mathcal{K}_{1}$. Then

$$
\mathcal{K}^{*}=\mathcal{V}_{\mathcal{K}} \times\left\langle t_{1}\right\rangle \times\left\langle t_{2}\right\rangle \times \mathcal{R}^{*}
$$

where the group of principal units $V_{\mathcal{K}}=1+M_{\mathcal{K}}$ and $\mathcal{R}^{*}=\mathcal{R} \backslash\{0\}$. From this observation we have the following,

Proposition 2.1.2. We can endow $\mathcal{K}^{*}$ with the product of the induced topology from $\mathcal{K}$ on the group $\mathcal{V}_{\mathcal{K}}$ and the discrete topology on $\left\langle t_{1}\right\rangle \times\left\langle t_{2}\right\rangle \times \mathcal{R}^{*}$. Moreover, every Cauchy sequence with respect to this topology converges in $\mathcal{K}^{*}$.

Proof. cf [7] Chapter 1: Higher dimensional local fields.

\section{$2.2 d$-dimensional local fields}

We will now generalize all the concepts introduce before to any dimension. In the rest of this section $E$ will denote a local field, $k_{E}$ its residue field and $\pi_{E}$ a uniformizer for $E$.

Definition 2.2.1. $\mathcal{K}$ is an d-dimensional local field, i.e., a field for which there is a chain of fields $\mathcal{K}_{d}=\mathcal{K}, \mathcal{K}_{d-1}, \ldots, \mathcal{K}_{0}$ such that $\mathcal{K}_{i+1}$ is a complete discrete valuation ring with residue field $\mathcal{K}_{i}, 0 \leq i \leq d-1$, and $\mathcal{K}_{0}$ is a finite field of characteristic $p$. 
If $k$ is a finite field then $\mathcal{K}=k\left(\left(T_{1}\right)\right) \ldots\left(\left(T_{d}\right)\right)$ is a $d$-dimensional local field with

$$
\mathcal{K}_{i}=k\left(\left(T_{1}\right)\right) \ldots\left(\left(T_{i}\right)\right) \quad 1 \leq i \leq d
$$

If $E$ is a local field, then $\mathcal{K}=E\left\{\left\{T_{1}\right\}\right\} \ldots\left\{\left\{T_{d-1}\right\}\right\}$ is defined inductively as

$$
E_{d-1}\left\{\left\{T_{d-1}\right\}\right\}
$$

where $E_{d-1}=E\left\{\left\{T_{1}\right\}\right\} \ldots\left\{\left\{T_{d-2}\right\}\right\}$. We have that $\mathcal{K}$ is a $d$-dimensional local field with residue field $\mathcal{K}_{d-1}=k_{E_{d-1}}\left(\left(T_{d-1}\right)\right)$, and by induction

$$
k_{E_{d-1}}=k_{E}\left(\left(T_{1}\right)\right) \ldots\left(\left(T_{d-2}\right)\right) \text {. }
$$

Therefore $\mathcal{K}_{d-1}=k_{E}\left(\left(T_{1}\right)\right) \ldots\left(\left(T_{d-1}\right)\right)$. These fields are called the standard fields.

From now on we will assume $\mathcal{K}$ has mixed characteristic, i.e., $\operatorname{char}(\mathcal{K})=0$ and $\operatorname{char}\left(\mathcal{K}_{d-1}\right)=p$. The following theorem classifies all such fields.

Theorem 2.2.1 (Classification Theorem). Let $\mathcal{K}$ be an $d$-dimensional local field of mixed characteristic. Then $\mathcal{K}$ is a finite extension of a standard field

$$
E\left\{\left\{T_{1}\right\}\right\} \ldots\left\{\left\{T_{d-1}\right\}\right\}
$$

where $E$ is a local field, and there is a finite extension of $\mathcal{K}$ which is a standard field.

Proof. cf. [7] $§ 1.1$ Classification Theorem.

Definition 2.2.2. An d-tuple of elements $t_{1}, \ldots, t_{d} \in \mathcal{K}$ is called a system of local 
parameters of $\mathcal{K}$, if $t_{d}$ is a prime in $\mathcal{K}_{d}, t_{d-1}$ is a unit in $\mathcal{O}_{\mathcal{K}}$ but its residue in $\mathcal{K}_{d-1}$ is a prime element of $\mathcal{K}_{d-1}$, and so on.

For the standard field $E\left\{\left\{T_{1}\right\}\right\} \ldots\left\{\left\{T_{d-1}\right\}\right\}$ we can take as a system of local parameters $t_{d}=\pi_{E}, t_{d-1}=T_{d-1}, \ldots, t_{1}=T_{1}$.

Definition 2.2.3. We define a discrete valuation of rank $d$ to be the map $v=$ $\left(v_{1}, \ldots, v_{d}\right): \mathcal{K}^{*} \rightarrow \mathbb{Z}^{d}, v_{d}=v_{\mathcal{K}_{d}}, v_{d-1}(x)=v_{\mathcal{K}_{d-1}}\left(x_{d-1}\right)$ where $x_{d-1}$ is the residue in $\mathcal{K}_{d-1}$ of $x t_{d}^{-v_{n}(x)}$, and so on.

Although the valuation depends, for $n>1$, on the choice of $t_{2}, \ldots, t_{d}$, it is independent in the class of equivalent valuations.

\subsection{Extensions of $\mathcal{K}$}

Let $\mathcal{L} / \mathcal{K}$ be a finite extension of the $d$-dimensional local field $\mathcal{K}$. Then $\mathcal{L}$ is also a $d$-dimensional local field.

Definition 2.3.1. Let $t_{1}, \ldots, t_{d}$ and $t_{1}^{\prime}, \ldots, t_{d}^{\prime}$ be a system of local parameters for $K$ and $L$, respectively, with associated valuations $v$ and $v^{\prime}$, respectively. Put

$$
E(\mathcal{L} / \mathcal{K})=\left(v_{j}^{\prime}\left(t_{i}\right)\right)_{i, j}=\left(\begin{array}{cccc}
e_{1} & 0 & \ldots & 0 \\
\ldots & e_{2} & \ldots & 0 \\
\ldots & \ldots & \ldots & 0 \\
\ldots & \ldots & \ldots & e_{d}
\end{array}\right)
$$

where $e_{i}=e\left(\mathcal{L}_{i} / \mathcal{K}_{i}\right), i=1, \ldots, n$. Then $e_{i}$ does not depend on the choice of the 
parameters, and $[\mathcal{L} / \mathcal{K}]=f(\mathcal{L} / \mathcal{K}) \prod_{i=1}^{n} e_{i}(\mathcal{L} / \mathcal{K})$, where $f(\mathcal{L} / \mathcal{K})=\left[\mathcal{L}_{0} / \mathcal{K}_{0}\right]$ (cf. [7] $\S 1.2)$.

\subsection{Topology on $\mathcal{K}$}

We define the topology on $E\left\{\left\{T_{1}\right\}\right\} \ldots\left\{\left\{T_{d-1}\right\}\right\}$ by induction on $d$. For $d=1$ we define the topology to be the topology of a one-dimensional local field. Suppose we have defined the topology on a standard $d$-dimensional local field $E_{d}$ and let $\mathcal{K}=E_{d}\{\{T\}\}$. Denote by $P_{E_{d}}(c)$ the set $\left\{x \in E_{d}: v_{E_{d}}(x) \geq c\right\}$. Let $\left\{V_{i}\right\}_{i \in \mathbb{Z}}$ be a sequence of neighborhoods of zero in $E_{d}$ such that

$$
\left\{\begin{array}{l}
\text { 1. there is a } c \in \mathbb{Z} \text { such that } P_{E_{d}}(c) \subset V_{i} \text { for all } i \in \mathbb{Z} \text {. } \\
\text { 2. for every } l \in \mathbb{Z} \text { we have } P_{E_{d}}(l) \subset V_{i} \text { for all sufficiently large } i \text {. }
\end{array}\right.
$$

and put $\mathcal{V}_{\left\{V_{i}\right\}}=\left\{\sum b_{i} T^{i}: b_{i} \in V_{i}\right\}$. These sets form a basis of neighborhoods of 0 for a topology on $\mathcal{K}$. For an arbitrary $d$-dimensional local field $L$ of mixed characteristic we can find, by the Classification Theorem, a standard field that is a finite extension of $L$ and we can give $L$ the topology induced by the standard field.

Proposition 2.4.1. Let $\mathcal{L}$ be a d-dimensional local field of mixed characteristic with the topology defined above.

1. $\mathcal{L}$ is complete with this topology. Addition is a continuous operation and multiplication by a fixed $a \in \mathcal{L}$ is a continuous map. 
2. Multiplication is a sequentially continuous map, i.e., if $x \in \mathcal{L}$ and $y_{k} \rightarrow y$ in $\mathcal{L}$ then $x y_{k} \rightarrow x y$.

3. This topology is independent of the choice of the standard field above $\mathcal{L}$.

4. If $\mathcal{K}$ is a standard field and $\mathcal{L} / \mathcal{K}$ is finite, then the topology above coincides with the natural vector space topology as a vector space over $\mathcal{K}$.

5. The reduction map $\mathcal{O}_{\mathcal{L}} \rightarrow k_{\mathcal{L}}=\mathcal{L}_{d-1}$ is continuous and open (where $\mathcal{O}_{\mathcal{L}}$ is given the subspace topology from $\mathcal{L}$, and $k_{\mathcal{L}}=\mathcal{L}_{d-1}$ the $(d-1)$-dimensional topology).

Proof. All the proofs can be found in [20] Theorem 4.10.

\subsection{Topology on $\mathcal{K}^{*}$}

Let $\mathcal{R} \subset \mathcal{K}=\mathcal{K}_{d}$ be a set of representatives of the last residue field $\mathcal{K}_{0}$. Let $t_{1}, \ldots, t_{d}$ be a fixed system of local parameters for $\mathcal{K}$, i.e., $t_{d}$ is a uniformizer for $\mathcal{K}, t_{d-1}$ is a unit in $\mathcal{O}_{\mathcal{K}}$ but its residue in $\mathcal{K}_{d-1}$ is a uniformizer element of $\mathcal{K}_{d-1}$, and so on. Then

$$
\mathcal{K}^{*}=\mathcal{V}_{\mathcal{K}} \times\left\langle t_{1}\right\rangle \times \cdots \times\left\langle t_{d}\right\rangle \times \mathcal{R}^{*}
$$

where the group of principal units $V_{\mathcal{K}}=1+M_{\mathcal{K}}$ and $\mathcal{R}^{*}=\mathcal{R} \backslash\{0\}$. From this observation we have the following, 
Proposition 2.5.1. We can endow $\mathcal{K}^{*}$ with the product of the induced topology from $\mathcal{K}$ on the group $\mathcal{V}_{\mathcal{K}}$ and the discrete topology on $\left\langle t_{1}\right\rangle \times \cdots \times\left\langle t_{d}\right\rangle \times \mathcal{R}^{*}$. In this topology we have,

1. Multiplication is sequentially continuous, i.e., if $a_{n} \rightarrow a$ and $b_{n} \rightarrow b$ then $a_{n} b_{n} \rightarrow a b$

2. Every Cauchy sequence with respect to this topology converges in $\mathcal{K}^{*}$.

Proof. cf [7] Chapter $1 \S 1.4 .2$. 


\section{Chapter 3}

\section{Formal groups}

In the rest of this paper $F$ will be a formal group over the ring of integers, $\mathcal{O}_{K}$, of a local field $K / \mathbb{Q}_{p}$. Let $\operatorname{End}_{\mathcal{O}_{K}}(F)$ be the ring of endomorphisms of $F$. For $t \in \operatorname{End}_{\mathcal{O}_{K}}(F)$ we denote $t^{\prime}(0)$ by $c(t)$. This induces an embedding

$$
c: \operatorname{End}_{\mathcal{O}_{K}}(F) \rightarrow \mathcal{O}_{K}
$$

We have that that $c\left(\operatorname{End}_{\mathcal{O}_{K}}(F)\right)$ is a closed subring of $\mathcal{O}_{K}$ ( cf. [14] §2.3). In particular $\mathbb{Z}_{p} \subset c\left(\operatorname{End}_{\mathcal{O}_{K}}(F)\right)$. Let $S / \mathbb{Q}_{p}$ be a local field with ring of integers $C$ such that $C \subset c\left(\operatorname{End}_{\mathcal{O}_{K}}(F)\right)$. Let us fix once and for all a uniformizer $\pi$ for $C$. Let $j$ be the degree of inertia of $S / \mathbb{Q}_{p}$, i.e., $\left|k_{S}\right|=p^{j}$. In what follows $f$ will denote the element in $\operatorname{End}_{\mathcal{O}_{K}}(F)$ such that $c(f)=\pi$. 


\subsection{The Weiertrass lemma}

Let $E$ be a discrete valuation field of zero characteristic with integer ring $\mathcal{O}_{E}$ and maximal ideal $\mu_{E}$.

Lemma 3.1.1 (Weierstrass lemma). Let $g=a_{0}+a_{1} X+\cdots \in \mathcal{O}_{E}[[X]]$ be such that $a_{0}, \ldots, a_{n-1} \in \mu_{E}, n \geq 1$, and $a_{n} \notin \mu_{E}$. Then there exist a unique monic polynomial $c_{0}+\cdots+X^{n}$ with coefficients in $\mu_{E}$ and a series $b_{0}+b_{1} X \cdots$ with coefficients in $\mathcal{O}_{E}$ and $b_{0}$ a unit, i.e., $b_{0} \neq \mu_{E}$, such that

$$
g=\left(c_{0}+\cdots+X^{n}\right)\left(b_{0}+b_{1} X \cdots\right)
$$

Proof. See [18] IV. $§ 9$ Theorem 9.2.

\subsection{The group $F\left(\mu_{\mathcal{M}}\right)$}

Let $\mathcal{K}$ be a $d$-dimensional local field containing the local field $K$. For example, we may consider $\mathcal{K}$ to be $K\left\{\left\{T_{1}\right\}\right\} \cdots\left\{\left\{T_{d-1}\right\}\right\}$. Denote by $F\left(\mu_{\mathcal{K}}\right)$ the group with underlying set $\mu_{\mathcal{K}}$ and operation defined by the formal group $F$. More generally, if $\mathcal{M}$ is an algebraic extension of $\mathcal{K}$ we define 


$$
F\left(\mu_{\mathcal{M}}\right):=\bigcup_{\mathcal{M} \supset \mathcal{L} \supset \mathcal{K} \mid[\mathcal{L} / \mathcal{K}]<\infty} F\left(\mu_{\mathcal{L}}\right) .
$$

An element $f \in \operatorname{End}(F)$ is said to be an isogeny if the map $f: F\left(\mu_{\overline{\mathcal{K}}}\right) \rightarrow F\left(\mu_{\overline{\mathcal{K}}}\right)$ induced by it is surjective with finite kernel.

If the reduction of $f$ in $k_{K}[[X]], k_{K}$ the residue field of $K$, is not zero then it is of the form $f_{1}\left(X^{p^{h}}\right)$ with $f_{1}^{\prime}(0) \in \mathcal{O}_{K}^{*}$, cf. [14] Proposition 1.1. In this case we say that $f$ has finite height. If on the other hand the reduction of $f$ is zero we say it has infinite height.

Proposition 3.2.1. $f$ is an isogeny if and only if $f$ has finite height. Moreover, in this situation $|\operatorname{ker} f|=p^{h}$.

Proof. If the height is infinite, the coefficients of $f$ are divisible by a uniformizer of the local field $K$, so $f$ cannot be surjective. Let $h<\infty$ and $x \in \mu_{\mathcal{L}}$ where $\mathcal{L}$ is a finite extension of $\mathcal{K}$. Consider the series $f-x$ and apply Lemma 3.1.1 with $E=\mathcal{L}$, i.e.,

$$
f-x=\left(c_{0}+\cdots+X^{p^{h}}\right)\left(b_{0}+b_{1} X+\cdots\right),
$$

where $c^{\prime}$ s $\in \mu_{\mathcal{L}}, b^{\prime}$ s $\in \mathcal{O}_{\mathcal{L}}$ and $b_{0} \in \mathcal{O}_{\mathcal{L}}^{*}$. Therefore the equation $f(X)=x$ is equivalent to the equation $c_{0}+\cdots+X^{p^{h}}=0$ and since the $c^{\prime}$ s $\in \mu_{\mathcal{L}}$ every root belongs to $\mu_{\overline{\mathcal{K}}}$. 
Moreover, the polynomial $P(X)=c_{0}+\cdots+X^{p^{h}}$ is separable because $f^{\prime}(X)=$ $\pi t(X), t(X)=1+\ldots$ is an invertible series and $f^{\prime}=P^{\prime}\left(b_{0}+b_{1} X+\cdots\right)+P\left(b_{0}+\right.$ $\left.b_{1} X+\cdots\right)^{\prime}$ so $P^{\prime}$ can not vanish at a zero of $P$. We conclude that $P$ has $p^{h}$ roots, i.e., $\mid$ ker $f \mid=p^{h}$.

Proposition 3.2.2. Denote by $j$ the degree of inertia of $S / \mathbb{Q}_{p}$ and by $h_{1}$ the height of $f=[\pi]_{F}$. Then $j$ divides $h_{1}$, namely $h_{1}=j h$. Let $\kappa_{n}$ be the kernel of $f^{(n)}$. Then

$$
\kappa_{n} \simeq\left(C / \pi^{n} C\right)^{h} \text { and } \quad \lim _{\longleftarrow} \kappa_{n} \simeq C^{h}
$$

as $C$-modules. This $h$ is called the height of the formal group with respect to $C=\mathcal{O}_{S}$.

Proof. cf. [14] Proposition 2.3.

Remark 3.2.1. Notice that since the coefficients of $F$ are in the local field $K$ then $\kappa_{n} \subset \bar{K}$ for all $n \geq 1$.

Let us fix once and for all a basis $\left\{e^{i}\right\}_{i=1}^{h}$ for $\lim _{\longleftarrow} \kappa_{n}$. Denote by $e_{n}^{i}$ the reduction of $e^{i}$ to $\kappa_{n}$. Clearly $\left\{e_{n}^{i}\right\}$ is a basis for $\kappa_{n}$.

Throughout $K_{n}$ will denote the one dimensional local field $K\left(\kappa_{n}\right)$. Suppose $M / K$ is a finite extension, then $M_{n}$ will denote the local field $M\left(\kappa_{n}\right)$. Let $\mathcal{M}$ and $\mathcal{M}_{n}$ denote, respectively,

$$
M\left\{\left\{T_{1}\right\}\right\} \cdots\left\{\left\{T_{d-1}\right\}\right\} \text { and } M_{n}\left\{\left\{T_{1}\right\}\right\} \cdots\left\{\left\{T_{d-1}\right\}\right\}
$$




\subsection{The logarithm of the formal group}

We define the logarithm of the formal group $F$ to be the series

$$
l_{F}=\int_{0}^{X} \frac{d X}{F_{X}(0, X)}
$$

Observe that since $F_{X}(0, X)=1+\cdots \in \mathcal{O}_{K}[[X]]^{*}$ then $l_{F}$ has the form

$$
X+\frac{a_{2}}{2} X^{2}+\cdots+\frac{a_{n}}{n} X^{n}+\cdots
$$

where $a_{i} \in \mathcal{O}_{K}$.

Proposition 3.3.1. Let E be a field of characteristic 0 that is complete with respect to a discrete valuation, $\mathcal{O}_{E}$ the valuation ring of $E$ with maximal ideal $\mu_{E}$ and valuation $v_{E}$. Consider a formal group $F$ over $\mathcal{O}_{E}$, then

1. The formal logarithm induces a homomorphism

$$
l_{F}: F\left(\mu_{E}\right) \rightarrow E
$$

with the additive group law on $E$.

2. The formal logarithm induces the isomorphism

$$
l_{F}: F\left(\mu_{E}^{r}\right) \stackrel{\sim}{\longrightarrow} \mu_{E}^{r}
$$


for all $r \geq\left[v_{E}(p) /(p-1)\right]+1$ and

$$
v_{E}(l(x))=v(x) \quad\left(\forall x \in \mu_{E}^{r}\right) .
$$

In particular, this holds for $\mu_{E, 1}=\left\{x \in E: v_{E}(x)>v_{E}(p) /(p-1)+1\right\}$.

Proof. [24] IV Theorem 6.4 and Lemma 6.3.

Lemma 3.3.1. Let $E$ and $v_{E}$ as in the previous proposition. Then

$$
v_{E}(n !) \leq \frac{(n-1) v_{E}(p)}{p-1}
$$

and $v_{E}\left(x^{n} / n !\right) \rightarrow \infty$ as $n \rightarrow \infty$ for $x \in \mu_{E, 1}$.

Proof. The first assertion can be found in [24] IV. Lemma 6.2. For the second one notice that

$$
\begin{aligned}
v_{E}\left(x^{n} / n !\right) & \geq n v_{E}(x)-v(n !) \\
& \geq n v_{E}(x)-(n-1) \frac{v_{E}(p)}{p-1} \\
& =v_{E}(x)+(n-1)\left(v_{E}(x)-\frac{v_{E}(p)}{p-1}\right) .
\end{aligned}
$$

Since we are assuming that $x \in \mu_{E, 1}$, i.e., $v_{E}(x)>v_{E}(p) /(p-1)$, then $v_{E}\left(x^{n} / n !\right) \rightarrow \infty$ as $n \rightarrow \infty$.

Lemma 3.3.2. Let $\mathcal{L}$ be a d-dimensional local field containing the local field $K$, $g(X)=a_{1} X+\frac{a_{2}}{2} X^{2}+\cdots+\frac{a_{n}}{n} X^{n}+\cdots$ and $h(X)=a_{1} X+\frac{a_{2}}{2 !} X^{2}+\cdots+\frac{a_{n}}{n !} X^{n}+\cdots$ with 
$a_{i} \in \mathcal{O}_{K}$. Then $g$ and $h$ define, respectively, maps $g: \mu_{\mathcal{L}} \rightarrow \mu_{\mathcal{L}}$ and $h: \mu_{\mathcal{L}, 1} \rightarrow \mu_{\mathcal{L}, 1}$ that are sequentially continuous in the Parshin topology.

Proof. We may assume $\mathcal{L}$ is a standard $d$-dimensional local field. Let $\mathcal{V}_{\left\{V_{i}\right\}}$ be a basic neighborhood of zero that we can consider to be a subgroup of $\mathcal{L}$, and let $c>0$ such that $P_{\mathcal{L}}(c) \subset \mathcal{V}_{\left\{V_{i}\right\}}$. If $x_{n} \in \mu_{\mathcal{L}}$ for all $n$, then there exists an $N_{1}>0$ such that $v_{\mathcal{L}}\left(x_{n}^{i} / i\right), v_{\mathcal{L}}\left(x^{i} / i\right)>c$ for all $i>N_{1}$ and all $n$; because $i v_{\mathcal{L}}\left(x_{n}\right)-v(i) \geq$ $i v_{\mathcal{L}}\left(x_{n}\right)-\log _{p}(i) \geq i-\log _{p}(i) \rightarrow \infty$ as $i \rightarrow \infty$. On the other hand, if $x_{n} \in \mu_{\mathcal{L}, 1}$ for all $n$, then there exists an $N_{2}>0$ such that $v_{\mathcal{L}}\left(x_{n}^{i} / i !\right), v_{\mathcal{L}}\left(x^{i} / i !\right)>c$ for all $i>N_{2}$ and all $n$ by Lemma 3.3.1. Then, for $N=\max \left\{N_{1}, N_{2}\right\}$, we have

$$
\sum_{i=N+1}^{\infty} a_{i} \frac{x_{n}^{i}-x^{i}}{i}, \sum_{i=N+1}^{\infty} a_{i} \frac{x_{n}^{i}-x^{i}}{i !} \in P_{\mathcal{L}}(c) \subset \mathcal{V}_{\left\{V_{j}\right\}}
$$

Now, since multiplication is sequentially continuous and $x_{n} \rightarrow x$ then

$$
\sum_{i=1}^{N} a_{i} \frac{x_{n}^{i}-x^{i}}{i} \rightarrow 0, \sum_{i=1}^{N} a_{i} \frac{x_{n}^{i}-x^{i}}{i !} \rightarrow 0 \quad \text { as } n \rightarrow \infty
$$

Thus for $n$ large enough we have that

$$
g\left(x_{n}\right)-g(x), h\left(x_{n}\right)-h(x)=\sum_{i=1}^{N}+\sum_{i=N+1}^{\infty} \in \mathcal{V}_{\left\{V_{i}\right\}} .
$$

Remark 3.3.1. In particular, log $: \mu_{\mathcal{L}} \rightarrow \mu_{\mathcal{L}}, l_{F}: \mu_{\mathcal{L}} \rightarrow \mu_{\mathcal{L}}$ and $\exp _{F}=l_{F}^{-1}: \mu_{\mathcal{L}, 1} \rightarrow$ $\mu_{\mathcal{L}, 1}$ are sequentially continuous. 


\section{Chapter 4}

\section{The Kummer Pairing}

\subsection{Higher-dimensional local class field theory}

\subsubsection{Milnor- $K$-groups and norms}

Definition 4.1.1. Let $R$ be a ring and $m \geq 0$. We denote by $K_{m}(R)$ the group

$$
\underbrace{R^{\times} \otimes_{\mathbb{Z}} \cdots \otimes_{\mathbb{Z}} R^{\times}}_{m-\text { times }} / I
$$

where $I$ is the subgroup of $\left(R^{\times}\right)^{\otimes m}$ generated by

$$
\left\{a_{1} \otimes \cdots \otimes a_{m}: a_{1}, \ldots, a_{m} \in R^{\times} \text {such that } a_{i}+a_{j}=1 \text { for some } i \neq j\right\}
$$

$K_{m}(R)$ is called the $m^{\text {th }}$ Milnor-K-group of $R$. The element $a_{1} \otimes \cdots \otimes a_{m}$ is denoted by $\left\{a_{1}, \ldots, a_{m}\right\}$.

We define the symbol map $\{$,$\} to be the map$

$$
R^{\times} \times \cdots \times R^{\times} \rightarrow K_{d}(R): \quad\left(a_{1}, \ldots, a_{d}\right) \rightarrow\left\{a_{1}, \ldots, a_{d}\right\} .
$$


If $A$ is any abelian group, a map $g:\left(R^{\times}\right)^{d} \rightarrow A$ which is multilinear and satisfies $g\left(a_{1}, \ldots, a_{d}\right)=0$, whenever $a_{i}+a_{j}=1$ for some $i \neq j$ is called an Steinberg map. Remark 4.1.1. It follows from the very definition that any Steinberg map $g:\left(R^{\times}\right)^{d} \rightarrow$ $A$ can be factored through the symbol map, i.e., there exist a homomorphism $g^{d}$ : $K_{d}(R) \rightarrow A$ such that

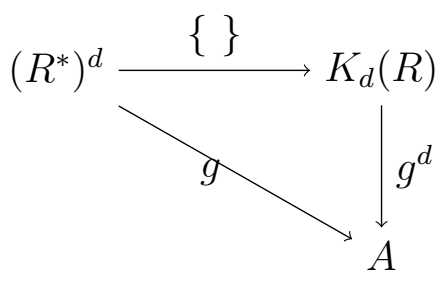

Proposition 4.1.1. The elements of the Milnor K-group satisfy the relations

1. $\left\{a_{1}, \ldots, a_{i}, \ldots,-a_{i}, \ldots, a_{m}\right\}=1$

2. $\left\{a_{1}, \ldots, a_{i}, \ldots, a_{j}, \ldots, a_{m}\right\}=\left\{a_{1}, \ldots, a_{j}, \ldots, a_{i}, \ldots, a_{m}\right\}^{-1}$

Proof. To simplify the notation we will assume $m=2$.

1. Noticing that $(1-a) /(1-1 / a)=-a$ it follows that

$$
\begin{aligned}
\{a,-a\} & =\{a, 1-a\}\{a, 1-1 / a\}^{-1} \\
& =\{a, 1-1 / a\}^{-1} \\
& =\{1 / a, 1-1 / a\} \\
& =1
\end{aligned}
$$


2. This follows immedeately from the previous item

$$
\begin{aligned}
\{a, b\}\{b, a\} & =\{-b, b\}\{a, b\}\{b, a\}\{-a, a\} \\
& =\{-a b, b\}\{-a b, a\} \\
& =\{-a b, a b\} \\
& =1
\end{aligned}
$$

From the definition we have $K_{1}(R)=R^{\times}$and we define $K_{0}(R):=\mathbb{Z}$. We also have a product

$$
K_{n}(R) \times K_{m}(R) \rightarrow K_{n+m}(R)
$$

where $\left\{a_{1}, \ldots, a_{n}\right\} \times\left\{a_{n+1}, \ldots, a_{n+m}\right\} \mapsto\left\{a_{1}, \ldots, a_{n+m}\right\}$.

It is possible to define a norm on the Milnor- $K$-groups:

Proposition 4.1.2. For each finite extension of fields $L / E$ there is a group homomorphism

$$
N_{L / E}: K_{m}(L) \rightarrow K_{m}(E),
$$

satisfying

1. When $m=1$ this maps coincides with the usual norm. 
2. For the tower $L / E_{1} / E_{2}$ of finite extensions we have $N_{L / E_{2}}=N_{E_{1} / E_{2}} \circ N_{L / E_{1}}$.

3. The composition $K_{m}(E) \rightarrow K_{m}(L) \stackrel{N_{L / E}}{\longrightarrow} K_{m}(E)$ coincides with multiplication by $[L / E]$.

4. If $\left\{a_{1}, \ldots, a_{m}\right\} \in K_{m}(L)$ with $a_{1}, \ldots, a_{i} \in L^{\times}$and $a_{i+1}, \ldots, a_{m} \in E^{\times}$, then

$$
N_{L / E}\left(\left\{a_{1}, \ldots, a_{m}\right\}\right)=N_{L / E}\left(\left\{a_{1}, \ldots, a_{i}\right\}\right)\left\{a_{i+1}, \ldots, a_{m}\right\} \in K_{m}(E),
$$

the right hand side is the product of a norm in $K_{i}(L)$ and a symbol in $K_{m-i}(E)$.

Proof. [6] IV and 8] 7.3.

Note in particular that if $a_{1} \in L^{\times}$and $a_{2}, \ldots, a_{m} \in E^{\times},(1)$ and (4) imply

$$
N_{L / E}\left(\left\{a_{1}, \ldots, a_{m}\right\}\right)=\left\{N_{L / E}\left(a_{1}\right), a_{2}, \ldots, a_{m}\right\}
$$

In the case where $L$ is a discrete valuation field there exist a boundary map between the Milnor K-group of $L$ and its residue field $k_{L}$ given in the following

Proposition 4.1.3. Suppose $L$ is a discrete valuation field with residue field $k_{L}$. There is a unique group homomorphism

$$
\partial: K_{m}(L) \rightarrow K_{m-1}\left(k_{L}\right)
$$

which satisfies

$$
\partial\left\{u_{1}, \ldots, u_{m-1}, \pi_{L}\right\}=\left\{\bar{u}_{1}, \ldots, \bar{u}_{m-1}\right\}
$$


for all uniformizers $\pi_{L} \in L^{*}$ and all $u_{i} \in \mathcal{O}_{L}^{*}$.

Proof. cf. [19] §2 Lemma 2.1.

Remark 4.1.2. Note that in the case $m=1$ the boundary map $\partial: L^{*}=K_{1}(L) \rightarrow$ $K_{0}\left(k_{L}\right)=\mathbb{Z}$ is clearly the valuation map $v: L^{*} \rightarrow \mathbb{Z}$.

Remark 4.1.3. In the case $\mathcal{L}=L\{\{T\}\}$, the composition of boundary maps

$$
K_{2}(L\{\{T\}\}) \stackrel{\partial}{\longrightarrow} K_{1}\left(k_{L}((T))\right) \stackrel{\partial}{\longrightarrow} K_{0}\left(k_{L}\right),
$$

sends the element $\{T, u\}, u \in L^{*}$, to $v_{L}(u)$. Indeed, let $\pi_{L}$ be any uniformizer for $L$, then since $T$ is a unit for the valuation field $L\{\{T\}\}$ we have $\partial\left(\left\{T, \pi_{L}\right\}\right)=T$. By $\operatorname{remark} 4.1 .2$ we have that $\partial(T)=v_{k_{L}((T))}(T)=1$. Thus $\partial\left(\partial\left(\left\{T, \pi_{L}\right\}\right)\right)=1=v_{L}\left(\pi_{L}\right)$. Since this is true for all uniformizers $\pi_{L}$ of $L$ and $\partial \circ \partial$ is a homomorphisms then the claim follows.

Definition 4.1.2. We endow $K_{d}(\mathcal{L})$ with the finest topology $\lambda_{d}$ for which the map

$$
\left(\mathcal{L}^{*}\right)^{\otimes d} \rightarrow K_{d}(\mathcal{L}): \quad\left(a_{1}, \ldots, a_{d}\right) \mapsto\left\{a_{1}, \ldots, a_{d}\right\}
$$

is sequentially continuous in each component with respect to the product topology on $\mathcal{L}^{*}$ and for which subtraction in $K_{d}(\mathcal{L})$ is sequentially continuous. Define

$$
K_{d}^{\text {top }}(\mathcal{L})=K_{d}(\mathcal{L}) / \Lambda_{d}(\mathcal{L})
$$


with the quotient topology where $\Lambda_{d}(\mathcal{L})$ denotes the intersection of all neighborhoods of 0 with respect to $\lambda_{d}$ (and so is a subgroup).

In [7] Chapter 6 Theorem 3, Fesenko proved that

$$
\Lambda_{d}(\mathcal{L})=\cap_{l \geq 1} l K_{d}(\mathcal{L})
$$

Proposition 4.1.4. Let $\mathcal{M} / \mathcal{L}$ be a finite extention, then norm $N_{\mathcal{M} / \mathcal{L}}: K_{d}(\mathcal{M}) \rightarrow$ $K_{d}(\mathcal{L})$ induces a norm

$$
N_{\mathcal{M} / \mathcal{L}}: K_{d}^{\text {top }}(\mathcal{M}) \rightarrow K_{d}^{\text {top }}(\mathcal{L}) .
$$

For this norm we have $N_{\mathcal{M} / \mathcal{L}}\left(\right.$ open subgroup) is open in $K_{d}^{\text {top }}(\mathcal{L})$. In particular, $N_{\mathcal{M} / \mathcal{L}}\left(K_{d}^{\text {top }}(\mathcal{M})\right)$ is open in $K_{d}^{\text {top }}(\mathcal{L})$.

Proof. Section 4.8, claims (1) and (2) of page 15 of [5].

\subsubsection{The reciprocity map}

Theorem 4.1.1 (A. Parshin, K. Kato). Let $\mathcal{L}$ be a d-dimensional local field. Then there exist a reciprocity map

$$
\Upsilon_{\mathcal{L}}: K_{d}(\mathcal{L}) \rightarrow \operatorname{Gal}\left(\mathcal{L}^{\mathrm{ab}} / \mathcal{L}\right),
$$

satisfying the properties

1. If $\mathcal{M} / \mathcal{L}$ is a finite extension of d-dimensional local fields then the following diagrams commute: 


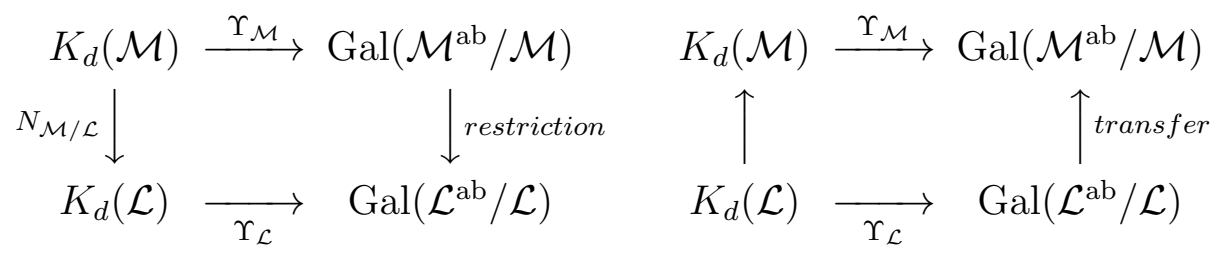

If moreover $\mathcal{M} / \mathcal{L}$ is abelian, then $\Upsilon_{\mathcal{L}}$ induces an isomorphism

$$
K_{d}(\mathcal{L}) / N_{\mathcal{M} / \mathcal{L}}\left(K_{d}(\mathcal{M})\right) \stackrel{\Upsilon_{\mathcal{L}}}{\longrightarrow} \operatorname{Gal}(\mathcal{M} / \mathcal{L})
$$

2. The map is compatible with the residue field $\overline{\mathcal{L}}$ of $\mathcal{L}$, i.e., the following diagram commutes:

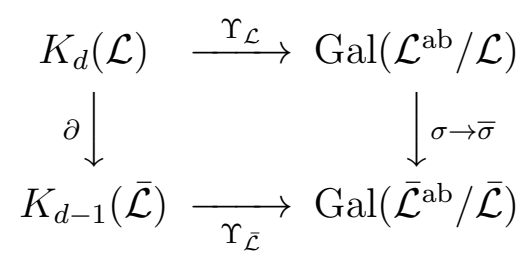

Here $\partial$ is the boundary map defined in Proposition 4.1.3.

3. The reciprocity map $\Upsilon_{\mathcal{L}}$ is sequentially continuous if we endow $K_{d}(\mathcal{L})$ with the topology $\lambda_{d}$ from definition 4.1.2.

Proof. The first two assertions can be found in [11] $\S 1$ Theorem 2. For the third one, let $\mathcal{M}$ be a finite abelian extension of $\mathcal{L}$, thus $\operatorname{Gal}\left(\mathcal{L}^{a b} / \mathcal{M}\right)$ is an open neighborhood of $G_{\mathcal{L}}^{a b}$. Let $x_{n}$ be a convergent sequence to the zero element of $K_{d}(\mathcal{L})$. Since $N_{\mathcal{M} / \mathcal{L}}\left(K_{d}^{t o p}(\mathcal{M})\right)$ is a open subgroup of $K_{d}^{\text {top }}(\mathcal{L})$ by Proposition 4.1.4, then

$$
\overline{x_{n}} \in N_{\mathcal{M} / \mathcal{L}}\left(K_{d}^{t o p}(\mathcal{M})\right) \quad(n>>0),
$$


where $\overline{x_{n}}$ is the image of $x_{n}$ in $K_{d}^{t o p}(\mathcal{L})$. Thus, there exist $y_{n} \in K_{d}(\mathcal{M})$ and $\beta_{n} \in$ $\Lambda_{m}(\mathcal{L})$ such that

$$
x_{n}=\beta_{n} N_{\mathcal{M} / \mathcal{L}}\left(y_{n}\right) \quad(n>>0) .
$$

From equation (4.1) we have that $\beta_{n} \in \cap_{l \geq 1} l K_{d}(\mathcal{L})$ which implies that $\Upsilon_{\mathcal{L}}\left(\beta_{n}\right)$ is the identity element in $G_{\mathcal{L}}^{a b}$ (because $G_{\mathcal{L}}^{a b}$ is a profinite group). Therefore

$$
\Upsilon_{\mathcal{L}}\left(x_{n}\right)=\Upsilon_{\mathcal{L}}\left(N_{\mathcal{M} / \mathcal{L}}\left(y_{n}\right)\right) \quad(n>>0),
$$

but the element on the right hand side of this equality is the identity on $\operatorname{Gal}\left(\mathcal{L}^{a b} / \mathcal{M}\right)$ by the second item of this Theorem. It follows that $\Upsilon_{\mathcal{L}}\left(x_{n}\right)$ converges to the identity elemenet of $G_{\mathcal{L}}^{a b}$.

\subsection{The pairing $(,)_{\mathcal{L}, n}$}

Let $\mathcal{L}$ be a $d$-dimensional local field containing $K$ and the group $\kappa_{n}$. We have the pairing

$$
(,)_{\mathcal{L}, n}: K_{d}(\mathcal{L}) \times F\left(\mu_{\mathcal{L}}\right) \rightarrow \kappa_{n}
$$

defined by $\left(\left\{a_{1}, \ldots, a_{d}\right\}, x\right)_{\mathcal{L}, n}=\Upsilon_{\mathcal{L}}\left(\left\{a_{1}, \ldots, a_{d}\right\}\right)(Z)-{ }_{F} Z$, where $f^{(n)}(Z)=x$ and $-{ }_{F}$ is the subtraction in the formal group $F$.

Proposition 4.2.1. The pairing just defined satisfies the following:

1. $(,)_{\mathcal{L}, n}$ is bilinear and $C$-linear on the right. 
2. The kernel on the right is $f^{(n)}\left(F\left(\mu_{\mathcal{L}}\right)\right)$.

3. $(a, x)_{\mathcal{L}, n}=0$ if and only if $a \in N_{\mathcal{L}(z) / \mathcal{L}}\left(K_{d}(\mathcal{L}(z))\right)$, where $f^{(n)}(z)=x$.

4. If $\mathcal{M} / \mathcal{L}$ is finite, $x \in F\left(\mu_{\mathcal{L}}\right)$ and $b \in K_{d}(\mathcal{M})$. Then

$$
(b, x)_{\mathcal{M}, n}=\left(N_{\mathcal{M} / \mathcal{L}}(b), x\right)_{\mathcal{L}, n}
$$

5. Let $L \supset \kappa_{m}, m \geq n$. Then

$$
(a, x)_{\mathcal{L}, n}=f^{(m-n)}\left((a, x)_{\mathcal{L}, m}\right)=\left(a, f^{(m-n)}(x)\right)_{\mathcal{L}, m}
$$

6. For a given $x \in K_{d}(\mathcal{L})$, the map

$$
K_{d}(\mathcal{L}) \rightarrow \kappa_{n}: a \mapsto(a, x)_{\mathcal{L}, n}
$$

is sequentially continuous.

7. Let $M$ be a finite extension of $L, a \in K_{d}(\mathcal{L})$ and $y \in F\left(\mu_{\mathcal{M}}\right)$. Then

$$
(a, y)_{\mathcal{M}, n}=\left(a, N_{\mathcal{M} / \mathcal{L}}^{F}(y)\right)_{\mathcal{M}, n}
$$

where $N_{\mathcal{M} / \mathcal{L}}^{F}(y)=\oplus_{\sigma} y^{\sigma}$, where $\sigma$ ranges over all embeddings of $M$ in $\bar{K}$ over L. Notice that such an embedding extends uniquely to an embedding from $\mathcal{M}=$ $M\{\{T\}\}$ over $\mathcal{L}=L\{\{T\}\}$. 
8. Let $t: F \rightarrow \tilde{F}$ be a isomorphism. Then

$$
(a, t(x))_{\mathcal{L}, n}^{\tilde{F}}=t\left((a, x)_{\mathcal{L}, n}^{F}\right)
$$

for all $a \in K_{d}(\mathcal{L}), x \in F\left(\mu_{\mathcal{L}}\right)$.

Proof. The first 5 properties and the last one follow from the definition of the pairing and Theorem 4.1.1.

The property 6 follows from the fact that the reciprocity map $\Upsilon_{\mathcal{L}}: K_{m}(\mathcal{L}) \rightarrow$ $\operatorname{Gal}\left(\mathcal{L}^{\mathrm{ab}} / \mathcal{L}\right)$ is sequentially continuous, here $K_{d}(\mathcal{L})$ is endowed with the finest topology for which the map

$$
\phi: \mathcal{L}^{* \oplus d} \rightarrow K_{d}(\mathcal{L})
$$

is sequentially continuous in each component with respect to the product topology on $\mathcal{L}^{*}$ and for which subtraction in $K_{d}(\mathcal{L})$ is sequentially continuous. Then for $z$ such that $f^{(n)}(z)=x$ consider the extension $\mathcal{L}(z) / \mathcal{L}$. The group $\operatorname{Gal}\left(\mathcal{L}^{a b} / \mathcal{L}(z)\right)$ is a neighborhood of $G_{\mathcal{L}}^{a b}$, so for any sequence $\left\{a_{m}\right\}$ coverging to zero in $K_{d}(\mathcal{L})$ we can take $m$ large enough such that $\Upsilon_{\mathcal{L}}\left(a_{m}\right) \in \operatorname{Gal}\left(\mathcal{L}^{a b} / \mathcal{L}(z)\right)$, that is $\Upsilon_{\mathcal{L}}\left(a_{m}\right)(z)=z$, so $\left(a_{m}, x\right)_{\mathcal{L}, n}=0$ for large enough $m$.

Finally, let us prove property 7 . Let $f^{(n)}(z)=y$ an take a finite Galois extension $\mathcal{N} \supset \mathcal{M}(z)$ over $\mathcal{L}$. Let $G=G(\mathcal{N} / \mathcal{L})$ and $H=G(\mathcal{N} / \mathcal{M}), w=[G: H]$, and $V: G / G^{\prime} \rightarrow H / H^{\prime}$ the transfer homomorphism. Let $g=\Upsilon_{\mathcal{L}}(a)$, then by Theorem 4.1.1 we have $V\left(\Upsilon_{\mathcal{L}}(a)\right)=\Upsilon_{\mathcal{M}}(a)$. The explicit computation of $V$ at $g \in G$ proceeds 
as follows (cf. [23] $\S 3.5$ ). Let $\left\{c_{i}\right\}$ be a set of representatives of for the right cosets of $H$ in $G$, i.e., $G=\sqcup H c_{i}$. Then for each $c_{i}, i=1, \ldots w$ there exist a $c_{j}$ such that $c_{i} g c_{j}^{-1}=h_{i} \in H$ and no two $c_{j}$ 's are equal; this is because $c_{i} g$ belongs to one and only one of the right cosets $H c_{j}$. Then $V(g)=\prod_{i=1}^{w} h_{i}$.

Also, notice that since $g c_{j}^{-1}=c_{i}^{-1} h_{i}$ then

$$
h_{i}(z) \ominus z=c_{i}^{-1}\left(h_{i}(z) \ominus z\right)=g\left(c_{j}^{-1}(z)\right) \ominus c_{i}^{-1}(z) .
$$

So we have

$$
\begin{aligned}
(a, y)_{\mathcal{M}, n} & =\Upsilon_{\mathcal{M}}(a)(z) \ominus z \\
& =V(g)(z) \ominus z \\
& =\left(\prod_{i=1}^{w} h_{i}\right) z \ominus z \\
& =\oplus_{i=1}^{w}\left(h_{i}(z) \ominus z\right) \\
& =\oplus_{i=1}^{w}\left(g\left(c_{j}^{-1}(z)\right) \ominus c_{i}^{-1}(z)\right) \\
& =g\left(\oplus_{i=1}^{w} c_{j}^{-1}(z)\right) \ominus\left(\oplus_{i=1}^{w} c_{j}^{-1}(z)\right) \\
& =\left(a, N_{\mathcal{M} / \mathcal{L}}^{F}(y)\right)_{\mathcal{L}, n}
\end{aligned}
$$

the last equality being true since $g=\Upsilon_{\mathcal{L}}(a)$ and $f^{(n)}\left(\oplus_{i=1}^{w} c_{j}^{-1}(z)\right)=\oplus_{i=1}^{w} c_{j}^{-1}(y)=$ $N_{\mathcal{M} / \mathcal{L}}^{F}(y)$ 
Let $\alpha$ denote the ramification index of $S$ over $\mathbb{Q}_{p}$. We say that a pair $(n, t)$ is admissible if there exist an integer $k$ such that $t-1-n \geq \alpha k \geq n$. For example, the pair $(n, 2 n+\alpha+1)$ is admissible with $k=[(n+\alpha / \alpha)]$. In the special case where $\alpha=1$, then $t-1-n \geq \alpha k \geq n$ becomes $t-1-n \geq k \geq n$. So in this case, the pair $(n, 2 n+1)$ is admissible with $k=n$.

The following proposition is vital for the main results in this paper. The idea of the proof was inspired by the proof of Proposition 4.1 in [14].

Proposition 4.2.2. Let $L \supset K_{t}=K\left(\kappa_{t}\right)$ with $(n, t)$ an admissible pair and let $\mathcal{L}=L\left\{\left\{T_{1}\right\}\right\} \cdots\left\{\left\{T_{d-1}\right\}\right\}$. For a given $\alpha \in K_{d}(\mathcal{L})$, the map

$$
F\left(\mu_{\mathcal{L}, 1}\right) \rightarrow \kappa_{n}: x \mapsto(\alpha, x)_{\mathcal{L}, n},
$$

is sequentially continuous, i.e., if $x_{k} \rightarrow x$ then $\left(\alpha, x_{k}\right)_{\mathcal{L}, n} \rightarrow(\alpha, x)_{\mathcal{L}, n}$. Here $F\left(\mu_{\mathcal{L}, 1}\right)$ is the set

$$
\left\{x \in \mathcal{L}: v_{\mathcal{L}}(x) \geq\left[\frac{v_{L}(p)}{p-1}\right]+1\right\}
$$

considered with the operation induced by the formal group $F$.

Remark: We will make the following two assumptions. First notice that it is enough to prove the result for $a \in \mathcal{L}^{*}$ such that $v_{\mathcal{L}}(a)=1$, because then it will be true for $\pi_{L}$ and $\pi_{L} u$ with $v_{\mathcal{L}}(u)=0$. Here $\pi_{L}$ a uniformizer for $L$.

Let $r$ be a $t$-normalized series, cf. $§ 4.3$. Notice also that we may assume that $r(X)=X$ is a $t$-normalized, otherwise we go to the isomorphic group law 
$r\left(F\left(r^{-1}(X), r^{-1}(Y)\right)\right)$.

Proof. We will drop the subscript $\mathcal{L}$ from the pairing notation and will make the two assumptions in the remark above, i.e., let $u \in \mathcal{L}^{*}$ with $v_{\mathcal{L}}(u)=1, b_{2} \ldots, b_{d} \in \mathcal{L}^{*}$ and $x \in F\left(\mu_{\mathcal{L}, 1}\right)$. Then

$$
\begin{aligned}
& \left(\left\{u, b_{2} \ldots, b_{d}\right\}, x\right)_{n}= \\
& \left(\left\{\frac{u}{u \oplus f^{(\alpha k+1)}(x)}, b_{2} \ldots, b_{d}\right\}, x\right)_{n} \oplus\left(\left\{u \oplus f^{(\alpha k+1)}(x), b_{2} \ldots, b_{d}\right\}, x\right)_{n} .
\end{aligned}
$$

We will show that the first term on the right hand side is zero and that the second side goes to zero when we take a sequence $\left\{x_{k}\right\}$ converging to zero. This would complete the proof.

Let us start with the second term. Let $m=n+\alpha k+1$. By (5) in the Proposition 4.2 .1 and the fact that $r(X)=X$, i.e., $\left(\left\{a, b_{2} \ldots, b_{d}\right\}, a\right)=0 \forall a \in F\left(\mu_{\mathcal{L}}\right)$, it follows 
that

$$
\begin{aligned}
& \left(\left\{u \oplus f^{(\alpha k+1)}(x), b_{2} \ldots, b_{d}\right\}, x\right)_{n}= \\
& =\left(\left\{u \oplus f^{(\alpha k+1)}(x), b_{2} \ldots, b_{d}\right\}, f^{(m-n)}(x)\right)_{m} \\
& =\left(\left\{u \oplus f^{(\alpha k+1)}(x), b_{2} \ldots, b_{d}\right\}, u \oplus f^{(\alpha k+1)}(x) \ominus u\right)_{m} \\
& =\left(\left\{u \oplus f^{(\alpha k+1)}(x), b_{2} \ldots, b_{d}\right\}, u \oplus f^{(\alpha k+1)}(x)\right)_{m} \oplus\left(\left\{u \oplus f^{(\alpha k+1)}(x), b_{2} \ldots, b_{d}\right\}, \ominus u\right)_{m} \\
& =\left(\left\{u \oplus f^{(\alpha k+1)}(x), b_{2} \ldots, b_{d}\right\}, \ominus u\right)_{m} \\
& =\left(\left\{u b^{-1}, b_{2} \ldots, b_{d}\right\}, \ominus u\right)_{m} \oplus\left(\left\{u \oplus f^{(\alpha k+1)}(x), b_{2} \ldots, b_{d}\right\}, \ominus u\right)_{m} \\
& =\left(\left\{\frac{u \oplus f^{(\alpha k+1)}(x)}{u}, b_{2} \ldots, b_{d}\right\}, \ominus u\right)_{m}
\end{aligned}
$$

Since $F(X, Y) \equiv X+Y(\bmod X Y)$, then $u \oplus f^{(\alpha k+1)}(x) \equiv u+f^{(\alpha k+1)}(x)\left(\bmod u f^{(\alpha k+1)}(x)\right)$ and so

$$
\frac{u \oplus f^{(\alpha k+1)}(x)}{u} \equiv 1+\frac{f^{(\alpha k+1)}(x)}{u} \quad\left(\bmod f^{(\alpha k+1)}(x)\right)
$$

But $v_{\mathcal{L}}(u)=1$, so this element is a principal unit and if we take a sequence $\left\{x_{i}\right\}_{i \geq 1}$ converging to zero in the Parshin topology then, as $f: \mu_{\mathcal{L}, 1} \rightarrow \mu_{\mathcal{L}, 1}$ is sequentially continuous in the Parshin topology by Lemma 3.3.2, this element goes to 1 and hence

$$
\left\{\frac{u \oplus f^{(\alpha k+1)}\left(x_{i}\right)}{u}, b_{2} \ldots, b_{d}\right\} \rightarrow\left\{1, b_{2} \ldots, b_{d}\right\}
$$

in the topology of $K_{2}(\mathcal{L})$. Notice that $\left\{1, b_{2} \ldots, b_{d}\right\}=\left\{1-b_{2}, b_{2} \ldots, b_{d}\right\}\left\{1, b_{2} \ldots, b_{d}\right\}=$ $\left\{1-b_{2}, b_{2} \ldots, b_{d}\right\}=\mathbf{1}$, where $\mathbf{1}$ is the unit element in $K_{2}(\mathcal{L})$. Then by $(6)$ in the 
Proposition 4.2 .1

$$
\left(\left\{\frac{u \oplus f^{(\alpha k+1)}\left(x_{i}\right)}{u}, b_{2} \ldots, b_{d}\right\}, \ominus u\right)_{m} \stackrel{i \rightarrow \infty}{\longrightarrow}(\mathbf{1}, \ominus u)_{m}=0
$$

Now we will show that first term on the right hand side of equation 4.2 is zero by showing that $\left(u \oplus f^{(\alpha k+1)}(x)\right) / u$ is a $p^{k}$ th power in $\mathcal{L}^{*}$, which is enough since $\pi^{n}$ divides $p^{k}$, because $n \leq \alpha k$. Indeed, since $x \in F\left(\mu_{\mathcal{L}, 1}\right)$ then by Proposition 3.3.1

$$
f^{(\alpha k+1)}(x)=l_{F}^{-1} \circ l_{F}\left(f^{(\alpha k+1)}(x)\right)=l_{F}^{-1}\left(\pi^{\alpha k+1} l_{F}(x)\right)=\pi^{\alpha k+1} w
$$

for some $w \in \mu_{\mathcal{L}, 1}$. Then equation 4.3 implies

$$
\frac{u \oplus f^{(\alpha k+1)}(x)}{u}=1+p^{k} w_{2}
$$

for $w_{2} \in \mu_{\mathcal{L}, 1}$, since $\pi^{\alpha k}=\epsilon p^{k}$ for some unit $\epsilon$. Then

$$
\log \left(\frac{u \oplus f^{(\alpha k+1)}(x)}{u}\right)=\log \left(1+p^{k} w_{2}\right)=p^{k} w_{3}
$$

where $w_{3} \in \mu_{\mathcal{L}, 1}$, so there exist a $w_{4} \in \mu_{\mathcal{L}, 1}$ such that $\log \left(1+w_{4}\right)=w_{3}$, thus

$$
\frac{u \oplus f^{(\alpha k+1)}(x)}{u}=\left(1+w_{4}\right)^{p^{k}}
$$

and so

$$
\left(\left\{\frac{u}{u \oplus f^{(\alpha k+1)}(x)}, b_{2} \ldots, b_{d}\right\}, x\right)_{n}=0
$$

This proves the theorem. 


\subsection{Norm Series}

A power series $r \in \mathcal{O}_{K}[[X]], r(0)=0, c(r) \in \mathcal{O}_{K}^{*}$ is called $n$-normalized if for every local field $L \supset \kappa_{n}, \mathcal{L}=L\left\{\left\{T_{1}\right\}\right\} \ldots\left\{\left\{T_{d-1}\right\}\right\}$, and all $1 \leq i \leq d$

$$
\left(\left\{a_{1}, \ldots, a_{i-1}, r(x), a_{i+1}, \ldots, a_{d}\right\}, x\right)_{\mathcal{L}, n}=0
$$

for all $x \in F\left(\mu_{\mathcal{L}}\right)$ and all $a_{1}, \ldots, a_{i-1}, a_{i+1}, \ldots, a_{d} \in \mathcal{L}^{*}$.

The following proposition will provide a way of constructing norm series.

Proposition 4.3.1. Let $g \in \mathcal{O}_{K}[[X]], g(0)=0$ and $c(g) \in \mathcal{O}_{K}^{*}$. The series $s=$ $\prod_{v \in \kappa_{n}} g(F(X, v))$ belongs to $\mathcal{O}_{K}[[X]]$ and has the form $r_{g}\left(f^{(n)}\right)$, where $r_{g} \in \mathcal{O}_{K}[[X]]$. Then, the series $r_{g}$ is $n$ - normalized and

$$
r_{g}^{\prime}(0)=\frac{\prod_{v \neq 0 \in \kappa_{n}} g(v)}{\pi^{n}} g^{\prime}(0)
$$

Proof. The proof follows closely [14] Proposition 3.1.

First, the coefficients of $s$ are in $\mathcal{O}_{K}$ because $s^{\sigma}=s$ for every $\sigma \in G_{K}=$ $\operatorname{Gal}(\bar{K} / K)$. Now, applying Lemma 3.1.1 to $s$ and $f^{(n)}$, we get $s=P s_{1}$ and $f^{(n)}=Q f_{1}$, where $P$ and $Q$ is a monic polynomials and $s_{1}, f_{1} \in \mathcal{O}_{K}[[X]]^{*}$. Since $s(F(X, v))=s(X)$ for all $v \in \kappa_{n}$, then $P(v)=0$ for all $v \in \kappa_{n}$ and so $Q=\prod_{v \in \kappa_{n}}(X-$ $v$ ) divides $P$. This implies that $s$ is divisible by $f^{(n)}$, i.e., $s=f^{(n)}\left(a_{0}+a_{1} X+\cdots\right)$. 
In particular,

$$
s-f^{(n)} \cdot a_{0}=f^{(n)} \cdot\left(a_{1} X+\cdots\right) .
$$

But from $s(F(X, v))=s(X)$ we see that $a_{1} X+\cdots$ must satisfy the same property and so $a_{1} v+\cdots=0$, for all $v \in \kappa_{n}$. Therefore this series is also divisible by $f^{(n)}$ and repeating the process we get $s=r_{g}\left(f^{(n)}\right)$. Let us compute now $c\left(r_{g}\right)$. Taking the logarithmic derivative on $\mathrm{s}$ and then multiplying by $X$ we get

$$
\frac{s^{\prime}(X)}{s(X)} X=\sum_{v \in \kappa_{n}} \frac{g^{\prime}(F(X, v)) F_{X}(X, v) X}{g(F(X, v))}
$$

which implies

$$
\frac{s^{\prime}(0)}{\prod_{0 \neq v \in \kappa_{n}} g(v)}=g^{\prime}(0)
$$

From $s^{\prime}=r_{g}^{\prime}\left(f^{(n)}\right) f^{(n)^{\prime}}$ we obtain

$$
r_{g}^{\prime}(0)=\frac{s^{\prime}(0)}{f^{(n)^{\prime}(0)}}=\frac{c(g) \prod_{0 \neq v \in \kappa_{n}} g(v)}{\pi^{n}} .
$$

Each $g(v)$ is associated to $v, 0 \neq v \in \kappa_{n}$, then $\prod_{0 \neq v \in \kappa_{n}} g(v)$ is associated to $\prod_{0 \neq v \in \kappa_{n}} v$, but the latter is associated to $\pi^{n}$ from the equation $f=P f_{1}$. Then $c\left(r_{g}\right) \in \mathcal{O}_{K}^{*}$. Finally, we will show that $\left(\left\{a_{1}, \ldots, a_{i-1}, r_{g}(x), a_{i+1}, \ldots, a_{d}\right\}, x\right)=0$. Let $L$ be a local field containing $\kappa_{n}, \mathcal{L}=L\left\{\left\{T_{1}\right\}\right\} \cdots\left\{\left\{T_{d-1}\right\}\right\}, x \in F\left(\mu_{\mathcal{L}}\right)$ and $z$ such that $f^{(n)}(z)=x$. Then

$$
r_{g}(x)=\prod_{v \in \kappa_{n}} g\left(z+{ }_{F} v\right)=\prod_{i} N_{\mathcal{L}(z) / \mathcal{L}}\left(g\left(z_{i}\right)\right)
$$


where the $z_{i}$ are pairwise non-conjugate over $\mathcal{L}$ distinct roots of $f^{(n)}(X)=x$, so

$$
\begin{aligned}
\left\{a_{1}, \ldots, a_{i-1}, r_{g}(x), a_{i+1}, \ldots, a_{d}\right\} & =\left\{a_{1}, \ldots, a_{i-1}, N_{\mathcal{L}(z) / \mathcal{L}}\left(\prod_{i} g\left(z_{i}\right)\right), a_{i+1}, \ldots, a_{d}\right\} \\
& =N_{\mathcal{L}(z) / \mathcal{L}}\left(\left\{a_{1}, \ldots, a_{i-1}, \prod_{i} g\left(z_{i}\right), a_{i+1}, \ldots, a_{d}\right\}\right)
\end{aligned}
$$

The last equality follows from Proposition 4.1.2 (1) and (4). The result now follows from Proposition 4.2.1. 


\section{Chapter 5}

\section{The maps $\psi$ and $\rho$}

\subsection{The generalized trace}

Let $E$ be a complete discrete valuation field. We define a map

$$
c_{E\{\{T\}\} / E}: E\{\{T\}\} \rightarrow E
$$

by $c_{E\{\{T\}\} / E}\left(\sum_{i \in \mathbb{Z}} a_{i} T^{i}\right)=a_{0}$. Let $\mathcal{E}=E\left\{\left\{T_{1}\right\}\right\} \ldots\left\{\left\{T_{d-1}\right\}\right\}$, we can define $c_{\mathcal{E} / E}$ by the composition

$$
c_{E\left\{\left\{T_{1}\right\}\right\} / E} \circ \cdots \circ c_{\mathcal{E} / E\left\{\left\{T_{1}\right\}\right\} \ldots\left\{\left\{T_{d-2}\right\}\right.} \cdot
$$

Lemma 5.1.1. This map satisfies the following properties

1. $c_{\mathcal{E} / E}$ is E-linear.

2. $c_{\mathcal{E} / E}(a)=a$, for all $a \in E$.

3. $c_{\mathcal{E} / E}$ is continuous with respect to the the Parshin topology on $\mathcal{E}$ and the discrete valuation topology on E. 
Proof. cf. [28] Lemma 2.1.

Suppose $L / S$ is a finite extension of local fields. Let $\pi$ be a uniformizer for $C=\mathcal{O}_{S}$ and let $\mathcal{L}=L\left\{\left\{T_{1}\right\}\right\} \ldots\left\{\left\{T_{d-1}\right\}\right\}$. The generalized trace $\mathbb{T}_{\mathcal{L} / S}$ is defined by the composition $\operatorname{Tr}_{L / S} \circ c_{\mathcal{L} / L}$ and this gives us the pairing

$$
\langle,\rangle: \mathcal{L} \times \mathcal{L} \rightarrow C,
$$

defined by $\langle x, y\rangle=\mathbb{T}_{\mathcal{L} / S}(x y)$. Let $\operatorname{Hom}_{C}^{c}(\mathcal{L}, S)$ and $\operatorname{Hom}_{C}^{\text {seq }}(\mathcal{L}, S)$ be the group of continuous and sequentially continuous, respectively, $C$-homomorphisms with respect to the Parshin topology on $\mathcal{L}$.

Proposition 5.1.1. We have an isomorphism of $C$-modules

$$
\mathcal{L} \stackrel{\sim}{\longrightarrow} \operatorname{Hom}_{C}^{\text {seq }}(\mathcal{L}, S): \alpha \mapsto\left(x \mapsto \mathbb{T}_{\mathcal{L} / S}(\alpha x)\right) .
$$

In particular, $\operatorname{Hom}_{C}^{\text {seq }}(\mathcal{L}, S)=\operatorname{Hom}_{C}^{c}(\mathcal{L}, S)$ since the generalized trace is continuous. Proof. Here we prove the case where the dimension $d$ is equal to 2, i.e., $\mathcal{L}=L\{\{T\}\}$. The general case is proved in the remark 5.1.1 after this proposition.

Let $\phi: \mathcal{L} \rightarrow S$ be a sequentially continuous $C$-linear map and define, for each $i \in \mathbb{Z}$, the map $\phi_{i}(x)=\phi\left(x T^{i}\right)$ for all $x \in L$. Then clearly $\phi_{i} \in \operatorname{Hom}_{C}(L, S)$ and this corresponds to the case $d=1$ for which we know that it exists an $a_{-i} \in L$ such that $\phi\left(x T^{i}\right)=\operatorname{Tr}_{L / S}\left(a_{-i} x\right)$ for all $x \in L$. Let $\alpha=\sum a_{i} T^{i}$, we must show that I. $\min \left\{v_{L}\left(a_{i}\right)\right\}>-\infty$. 
II. $v_{L}\left(a_{-i}\right) \rightarrow \infty$ as $i \rightarrow \infty$ (i.e., conditions (I) and (II) imply that $\alpha \in \mathcal{L}$ ).

III. $\phi(x)=\mathbb{T}_{\mathcal{L} / S}(\alpha x), \forall x \in \mathcal{L}$.

For any $x=\sum x_{i} T^{i} \in \mathcal{L}$ we have, by the sequential continuity of $\phi$ that

$$
\phi(x)=\sum_{i \in \mathbb{Z}} \phi\left(x_{i} T^{i}\right)=\sum_{i \in \mathbb{Z}} \operatorname{Tr}_{L / S}\left(a_{-i} x_{i}\right) .
$$

Suppose (I) was not true, then there exist a subsequence $\left\{a_{n_{k}}\right\}$ such that $v_{L}\left(a_{n_{k}}\right) \rightarrow$ $-\infty$ as $n_{k} \rightarrow \infty$ or as $n_{k} \rightarrow-\infty$. In the first case we take an $x=\sum x_{i} T^{i} \in \mathcal{L}$ such that $x_{i}$ is equal to $1 / a_{n_{k}}$ if $i=-n_{k}$ and 0 if $i \neq-n_{k}$. So $a_{-i} x_{i}=1$ if $i=-n_{k}$ and 0 if $i \neq-n_{k}$. Then the sum on the right of $(5.2)$ would not converge. In the second case we take $x_{i}$ to be equal to $1 / a_{n_{k}}$ if $i=-n_{k}$ and 0 if $i \neq-n_{k}$. So $a_{-i} x_{i}=1$ if $i=-n_{k}$ and 0 if $i \neq-n_{k}$ and again the sum on the right would not converge.

Suppose (II) was not true. Then $v_{E}\left(a_{n_{k}}\right)<M$ for some positive integer $M$ and a of negative integers $n_{k} \rightarrow-\infty$. Then take $x=\sum x_{i} T^{i} \in \mathcal{L}$ such that $x_{i}$ is equal to $1 / a_{n_{k}}$ for $i=-n_{k}$ and 0 for $i \neq-n_{k}$. So $a_{-i} x_{i}=1$ if $i=-n_{k}$ and 0 if $i \neq-n_{k}$ and the sum on the right of 5.2 would not converge.

Finally, (III) follows by noticing that by (I) and (II) the sum $\sum_{i \in \mathbb{Z}} a_{-i} x_{i}$ converges and

$$
\sum_{i \in \mathbb{Z}} \mathbb{T}_{E / S}\left(a_{-i} x_{i}\right)=\mathbb{T}_{E / S}\left(\sum_{i \in \mathbb{Z}} a_{-i} x_{i}\right)=\mathbb{T}_{\mathcal{L} / S}(x \alpha),
$$

since $\mathbb{T}_{E / S} \circ c_{\mathcal{L} / E}=\mathbb{T}_{\mathcal{L} / S}$. 
Remark 5.1.1. We include the proof of the general case of the proposition above.

Proof. The proof is done by induction in $d$. If $d=1$ the result is known. Suppose the result is true for $d \geq 1$ and let $\mathcal{L}=E\left\{\left\{T_{d}\right\}\right\}$ where $E=L\left\{\left\{T_{1}\right\}\right\} \cdots\left\{\left\{T_{d-1}\right\}\right\}$.

Let $\phi: \mathcal{L} \rightarrow S$ be a sequentially continuous $C$-linear map and define, for each $i \in \mathbb{Z}$, the sequentially continuous map $\phi_{i}(x)=\phi\left(x T_{d}^{i}\right)$ for all $x \in E$. Then clearly $\phi_{i} \in \operatorname{Hom}_{C}(E, S)$ and by the induction hypothesis we know that there exists an $a_{-i} \in E$ such that $\phi\left(x T_{d}^{i}\right)=\mathbb{T}_{E / S}\left(a_{-i} x\right)$ for all $x \in E$. Let $\alpha=\sum a_{i} T_{d}^{i}$, we must show that

I. $\min \left\{v_{E}\left(a_{i}\right)\right\}>-\infty$.

II. $v_{E}\left(a_{-i}\right) \rightarrow \infty$ as $i \rightarrow \infty$ (i.e., conditions (I) and (II) imply that $\alpha \in \mathcal{L}$ ).

III. $\phi(x)=\mathbb{T}_{\mathcal{L} / S}(\alpha x), \forall x \in \mathcal{L}$.

For any $x=\sum x_{i} T^{i} \in \mathcal{L}$ we have, by the sequential continuity of $\phi$ that

$$
\phi(x)=\sum_{i \in \mathbb{Z}} \phi\left(x_{i} T_{d}^{i}\right)=\sum_{i \in \mathbb{Z}} \mathbb{T}_{E / S}\left(a_{-i} x_{i}\right) .
$$

Suppose (I) was not true, then there exist a subsequence $\left\{a_{n_{k}}\right\}$ such that $v_{E}\left(a_{n_{k}}\right) \rightarrow$ $-\infty$ as $n_{k} \rightarrow \infty$ or as $n_{k} \rightarrow-\infty$. In the first case we take an $x=\sum x_{i} T_{d}^{i} \in \mathcal{L}$ such that $x_{i}$ is equal to $1 / a_{n_{k}}$ if $i=-n_{k}$ and 0 if $i \neq-n_{k}$. So $a_{-i} x_{i}=1$ if $i=-n_{k}$ and 0 if $i \neq-n_{k}$. Then the sum on the right of (5.3) would not converge. In the second case 
we take $x_{i}$ to be equal to $1 / a_{n_{k}}$ if $i=-n_{k}$ and 0 if $i \neq-n_{k}$. So $a_{-i} x_{i}=1$ if $i=-n_{k}$ and 0 if $i \neq-n_{k}$ and again the sum on the right would not converge.

Suppose (II) was not true. Then $v_{L}\left(a_{n_{k}}\right)<M$ for some positive integer $M$ and a of negative integers $n_{k} \rightarrow-\infty$. Then take $x=\sum x_{i} T_{d}^{i} \in \mathcal{L}$ such that $x_{i}$ is equal to $1 / a_{n_{k}}$ for $i=-n_{k}$ and 0 for $i \neq-n_{k}$. So $a_{-i} x_{i}=1$ if $i=-n_{k}$ and 0 if $i \neq-n_{k}$ and the sum on the right of 5.3 would not converge.

Finally, (III) follows by noticing that by (I) and (II) the sum $\sum_{i \in \mathbb{Z}} a_{-i} x_{i}$ converges and

$$
\sum_{i \in \mathbb{Z}} \operatorname{Tr}_{L / S}\left(a_{-i} x_{i}\right)=\operatorname{Tr}_{L / S}\left(\sum_{i \in \mathbb{Z}} a_{-i} x_{i}\right)=\mathbb{T}_{\mathcal{L} / S}(x \alpha) .
$$

Let $\mathcal{L}=L\left\{\left\{T_{1}\right\}\right\} \ldots\left\{\left\{T_{d-1}\right\}\right\}$ where $L$ is a local field. Let $v_{L}$ and $v_{\mathcal{L}}$ denote the valuations for $L$ and $\mathcal{L}$ respectively. Consider

$$
\mu_{\mathcal{L}, 1}:=\left\{x \in \mathcal{L}: v_{\mathcal{L}}(x) \geq\left[\frac{v_{L}(p)}{p-1}\right]+1\right\}
$$

and

$$
\mu_{L, 1}:=\mu_{\mathcal{L}, 1} \cap L=\left\{x \in L: v_{L}(x) \geq\left[\frac{v_{L}(p)}{p-1}\right]+1\right\},
$$

both with the additive structure, and denote by $R_{\mathcal{L}, 1}$ the dual of $\mu_{\mathcal{L}, 1}$ with respect 
to the pairing (5.1), i.e.,

$$
R_{\mathcal{L}, 1}:=\left\{x \in \mathcal{L}: \mathbb{T}_{\mathcal{L} / S}\left(x \mu_{\mathcal{L}, 1}\right) \subset C\right\}
$$

Let us show that

$$
R_{\mathcal{L}, 1}=\left\{x \in \mathcal{L}: v_{\mathcal{L}}(x) \geq-v_{L}(D(L / S))-\left[\frac{v_{L}(p)}{p-1}\right]-1\right\}
$$

where $D(L / S)$ is the different of the extension $L / S$. Indeed, for $d=1$ this is proven in [14] $\S 4.1$. Suppose it is true for $d \geq 1$, and let $\mathcal{L}=E_{d}\left\{\left\{T_{d}\right\}\right\}$, where $E_{d}=L\left\{\left\{T_{1}\right\}\right\} \cdots\left\{\left\{T_{d-1}\right\}\right\}$. If $x=\sum_{i \in \mathbb{Z}} x_{i} T_{d}^{i} \in R_{\mathcal{L}, 1}$, then since $\mu_{E_{d}, 1} \subset \mu_{\mathcal{L}, 1}$ we have that also $\mu_{E_{d}, 1} T_{d}^{-i} \subset \mu_{\mathcal{L}, 1}$ and

$$
\mathbb{T}_{\mathcal{L} / S}\left(x \mu_{E_{d}, 1} T_{d}^{-i}\right) \subset C,
$$

which implies $\mathbb{T}_{E_{d} / S}\left(x_{i} \mu_{E_{d}, 1}\right) \subset C$, since $\mathbb{T}_{\mathcal{L} / S}=\mathbb{T}_{E_{d} / S} \circ c_{\mathcal{L} / E_{d}}$. By induction hypothesis we have $v_{E_{d}}\left(x_{i}\right) \geq-v_{L}(D(L / S))-\left[\frac{v_{L}(p)}{p-1}\right]-1$ for all $i \in \mathbb{Z}$, therefore

$$
v_{\mathcal{L}}(x)=\min v_{E_{d}}\left(x_{i}\right) \geq-v_{L}(D(L / S))-\left[\frac{v_{L}(p)}{p-1}\right]-1 .
$$

Conversely, if $v_{\mathcal{L}}(x)=\min v_{E_{d}}\left(x_{i}\right) \geq-v_{L}(D(L / S))-\left[\frac{v_{L}(p)}{p-1}\right]-1$, then $v_{E_{d}}\left(x_{i}\right) \geq$ $-v_{L}(D(L / S))-\left[\frac{v_{L}(p)}{p-1}\right]-1$ for all $i \in \mathbb{Z}$. Then, by the induction hypothesis $\mathbb{T}_{E_{d} / S}\left(x_{i} \mu_{E_{d}, 1}\right) \subset C$ for all $i \in Z$, and therefore

$$
\mathbb{T}_{\mathcal{L} / S}\left(x \mu_{\mathcal{L}, 1}\right)=\sum_{i \in \mathbb{Z}} \mathbb{T}_{\mathcal{L} / S}\left(x_{i} T_{d}^{i} \mu_{\mathcal{L}, 1}\right)=\sum_{i \in \mathbb{Z}} \mathbb{T}_{E_{d} / S}\left(x_{i} \mu_{E_{d}, 1}\right) \subset C
$$


Thus, identity (5.4) holds.

Let

$$
R_{L, 1}:=R_{\mathcal{L}, 1} \cap L=\left\{x \in L: v_{L}(x) \geq-v_{L}(D(L / S))-\left[\frac{v_{L}(p)}{p-1}\right]-1\right\}
$$

Lemma 5.1.2. We have the isomorphism

$$
R_{\mathcal{L}, 1} / \pi^{n} R_{\mathcal{L}, 1} \stackrel{\sim}{\longrightarrow} \operatorname{Hom}_{C}^{\text {seq }}\left(\mu_{\mathcal{L}, 1}, C / \pi^{n} C\right)
$$

defined by

$$
\alpha \mapsto\left(x \mapsto \mathbb{T}_{\mathcal{L} / S}(\alpha x)\right)
$$

In particular, $\operatorname{Hom}_{C}^{\text {seq }}\left(\mu_{\mathcal{L}, 1}, C / \pi^{n} C\right)=\operatorname{Hom}_{C}^{c}\left(\mu_{\mathcal{L}, 1}, C / \pi^{n} C\right)$.

Proof. The proof is similar to that of Proposition 5.1.1. As in the proof of that proposition we will consider the case $d=2$, i.e., $\mathcal{L}=L\{\{T\}\}$, and leave the general case as a remark 5.1 .2 after the proof of this proposition. Take $\Phi \in \operatorname{Hom}_{C}^{\text {seq }}\left(\mu_{\mathcal{L}, 1}, C / \pi^{n} C\right)$ and let $\Phi_{i}\left(x_{i}\right)=\Phi\left(x_{i} T^{i}\right)$ for all $x_{i} \in \mu_{L, 1}$. Then $\Phi_{i} \in \operatorname{Hom}_{C}^{c}\left(\mu_{L, 1}, C / \pi^{n} C\right)$ and so there exist $\bar{a}_{-i} \in R_{L, 1} / \pi^{n} R_{L, 1}$ such that

$$
\Phi_{i}\left(x_{i}\right)=\operatorname{Tr}_{L / S}\left(\overline{a_{-i}} x_{i}\right) .
$$

Let $a_{-i} \in R_{L, 1}$ be a representative of $\bar{a}_{-i}$. Thus for $x=\sum x_{i} T^{i} \in \mu_{\mathcal{L}, 1}$, the sequential continuity of $\Phi$ implies 


$$
\Phi(x)=\sum_{i \in \mathbb{Z}} \Phi\left(x_{i} T^{i}\right)=\sum_{i \in \mathbb{Z}} \operatorname{Tr}_{L / S}\left(a_{-i} x_{i}\right) \quad\left(\bmod \pi^{n} C\right) .
$$

Let $\alpha=\sum a_{i} T^{i}$ and denote by $u_{i}$ the unit $a_{i} / \pi_{L}^{v_{L}\left(a_{i}\right)}$. We must show that

I. $\min \left\{v_{L}\left(a_{i}\right)\right\}>-\infty$.

II. $v_{L}\left(a_{-i}\right) \geq v_{L}\left(\pi^{n} R_{L, 1}\right)$ as $i \rightarrow \infty$ ( i.e., conditions 1 and 2 imply that $\alpha \in$ $\left.R_{\mathcal{L}, 1} / \pi^{n} R_{\mathcal{L}, 1}\right)$.

III. $\Phi(x)=\mathbb{T}_{\mathcal{L} / S}(\alpha x)\left(\bmod \pi^{n} C\right), \forall x \in \mu_{\mathcal{L}, 1}$.

Condition (I) follows immediately since $a_{-i} \in R_{L, 1}$, i.e., by equation (5.4)

$$
v_{L}\left(a_{-i}\right) \geq-\left(\left[\frac{v_{L}(p)}{p-1}\right]+1+v_{L}(D(L / S))\right) \quad \forall i \in \mathbb{Z} .
$$

Suppose condition (II) was not true. Instead of passing to a subsequence we may assume for simplicity that $v_{L}\left(a_{-i}\right)<v_{L}\left(\pi^{n} R_{L, 1}\right)$ for all $i \geq 0$. Let

$$
x=y\left(\pi_{L}^{\delta_{0}} u_{0}^{-1}+\pi_{L}^{\delta_{1}} u_{1}^{-1} T+\pi_{L}^{\delta_{2}} u_{2}^{-1} T^{2}+\cdots\right),
$$

where $u_{i}=a_{i} / \pi_{L}^{v_{L}\left(a_{i}\right)}$ and

$$
\delta_{i}=v_{L}\left(\pi^{n} R_{L, 1}\right)-v_{L}\left(a_{-i}\right)+\left[v_{L}(p) /(p-1)\right] \quad\left(\geq\left[v_{L}(p) /(p-1)\right]+1\right),
$$

for $i \geq 0$ and $y \in \mathcal{O}_{L}$ is arbitrary. Then $x \in \mu_{\mathcal{L}, 1}$ and $a_{-i} x_{i}=\pi_{L}^{w} y$ for $i \geq 0$, where $w=v_{L}\left(\pi^{n} R_{L, 1}\right)+\left[v_{L}(p) /(p-1)\right]$. The convergence of the right hand side of 5.5) 
would imply that

$$
\operatorname{Tr}_{L / S}\left(\pi_{L}^{w} y\right) \in \pi^{n} C \quad \forall y \in \mathcal{O}_{L}
$$

Thus $\pi_{L}^{w} / \pi^{n} \in D(L / S)^{-1}$, which implies $w \geq v_{L}\left(\pi^{n}\right)-v_{L}(D(L / S))$, that is,

$$
v_{L}\left(R_{L, 1}\right) \geq-v_{L}(D(L / S))-\left[v_{L}(p) /(p-1)\right],
$$

which is a contradiction since $v_{L}\left(R_{L, 1}\right)=-v_{L}(D(L / S))-\left[v_{L}(p) /(p-1)\right]-1$. Finally, condition (III) immediately follows from equation (5.5).

Remark 5.1.2. Next we will include the proof in the general case of the proposition above.

Proof. The proof is done by induction in $d$. If $d=1$ the result is known. Suppose the result is true for $d \geq 1$ and let $\mathcal{L}=E\left\{\left\{T_{d}\right\}\right\}$ where $E=L\left\{\left\{T_{1}\right\}\right\} \cdots\left\{\left\{T_{d-1}\right\}\right\}$.

Take $\Phi \in \operatorname{Hom}_{C}^{\text {seq }}\left(\mu_{\mathcal{L}, 1}, C / \pi^{n} C\right)$ and let $\Phi_{i}\left(x_{i}\right)=\Phi\left(x_{i} T_{d}^{i}\right)$ for all $x_{i} \in \mu_{E, 1}$. Then $\Phi_{i} \in \operatorname{Hom}_{C}^{c}\left(\mu_{E, 1}, C / \pi^{n} C\right)$ and so by the induction hypothesis there exists $\bar{a}_{-i} \in$ $R_{E, 1} / \pi^{n} R_{E, 1}$ such that

$$
\Phi_{i}\left(x_{i}\right)=\mathbb{T}_{E / S}\left(\overline{a_{-i}} x_{i}\right) .
$$

Let $a_{-i} \in R_{E, 1}$ be a representative of $\bar{a}_{-i}$. Thus for $x=\sum x_{i} T_{d}^{i} \in \mu_{\mathcal{L}, 1}$, the sequential continuity of $\Phi$ implies

$$
\Phi(x)=\sum_{i \in \mathbb{Z}} \Phi\left(x_{i} T_{d}^{i}\right)=\sum_{i \in \mathbb{Z}} \mathbb{T}_{E / S}\left(a_{-i} x_{i}\right) \quad\left(\bmod \pi^{n} C\right) .
$$


Let $\alpha=\sum a_{i} T_{d}^{i}$ and denote by $u_{i}$ the unit $a_{i} / \pi_{L}^{v_{E}\left(a_{i}\right)}$. We must show that

I. $\min \left\{v_{E}\left(a_{i}\right)\right\}>-\infty$.

II. $v_{E}\left(a_{-i}\right) \geq v_{E}\left(\pi^{n} R_{E, 1}\right)$ as $i \rightarrow \infty$ ( i.e., conditions 1 and 2 imply that $\alpha \in$ $\left.R_{\mathcal{L}, 1} / \pi^{n} R_{\mathcal{L}, 1}\right)$.

III. $\Phi(x)=\mathbb{T}_{\mathcal{L} / S}(\alpha x)\left(\bmod \pi^{n} C\right), \forall x \in \mu_{\mathcal{L}, 1}$.

Condition (I) follows immediately since $a_{-i} \in R_{E, 1}$, i.e., by equation (5.4)

$$
v_{E}\left(a_{-i}\right) \geq-\left(\left[\frac{v_{L}(p)}{p-1}\right]+1+v_{L}(D(L / S))\right) \quad \forall i \in \mathbb{Z}
$$

Suppose condition (II) was not true. Instead of passing to a subsequence we may assume for simplicity that $v_{E}\left(a_{-i}\right)<v_{E}\left(\pi^{n} R_{E, 1}\right)$ for all $i \geq 0$. Let

$$
x=y\left(\pi_{L}^{\delta_{0}} u_{0}^{-1}+\pi_{L}^{\delta_{1}} u_{1}^{-1} T_{d}+\pi_{L}^{\delta_{2}} u_{2}^{-1} T_{d}^{2}+\cdots\right),
$$

where $u_{i}=a_{i} / \pi_{L}^{v_{E}\left(a_{i}\right)}$ and

$$
\delta_{i}=v_{E}\left(\pi^{n} R_{E, 1}\right)-v_{E}\left(a_{-i}\right)+\left[v_{L}(p) /(p-1)\right] \quad\left(\geq\left[v_{L}(p) /(p-1)\right]+1\right),
$$

for $i \geq 0$ and $y \in \mathcal{O}_{L}$ is arbitrary. Then $x \in \mu_{\mathcal{L}, 1}$ and $a_{-i} x_{i}=\pi_{L}^{w} y$ for $i \geq 0$, where $w=v_{E}\left(\pi^{n} R_{E, 1}\right)+\left[v_{L}(p) /(p-1)\right]$. The convergence of the right hand side of (5.6) and the fact that $\mathbb{T}_{E / S}\left(\pi_{L}^{w}\right)=\operatorname{Tr}_{L / S}\left(\pi_{L}^{w} y\right)$ would imply that

$$
\operatorname{Tr}_{L / S}\left(\pi_{L}^{w} y\right) \in \pi^{n} C \quad \forall y \in \mathcal{O}_{L}
$$


Thus $\pi_{L}^{w} / \pi^{n} \in D(L / S)^{-1}$, which implies $w \geq v_{L}\left(\pi^{n}\right)-v_{L}(D(L / S))$, that is,

$$
v_{E}\left(R_{E, 1}\right) \geq-v_{L}(D(L / S))-\left[v_{L}(p) /(p-1)\right],
$$

which is a contradiction since $v_{E}\left(R_{E, 1}\right)=-v_{L}(D(L / S))-\left[v_{L}(p) /(p-1)\right]-1$ by (5.4).

Finally, condition (III) immediately follows from equation (5.3).

Let $T_{\mathcal{L}}$ be the image of $\mu_{\mathcal{L}}$ under the formal logarithm. This is a $C$-submodule of $\mathcal{L}$ such that $T_{\mathcal{L}} S=\mathcal{L}$. Indeed, let $x \in \mathcal{L}$ and take $n$ large enough such that $\pi^{n} x \in \mu_{\mathcal{L}, 1}$, then by Proposition 3.3 .1 there exist a $y \in F\left(\mu_{\mathcal{L}, 1}\right)$ such that $\pi^{n} x=l_{F}(y)$, thus $x \in T_{\mathcal{L}} S$. Let $R_{\mathcal{L}}$ be the dual of $T_{\mathcal{L}}$ with respect to the trace pairing $\mathbb{T}_{\mathcal{L} / S}$, then by Proposition 5.1.1 and by $\mathcal{L}=T_{\mathcal{L}} S$ we have the isomorphism

$$
R_{\mathcal{L}} \simeq \operatorname{Hom}_{C}^{\text {seq }}\left(T_{\mathcal{L}}, C\right) .
$$

We also have the following

Lemma 5.1.3. The generalized trace induces an injective homomorphism

$$
\begin{aligned}
R_{\mathcal{L}} / \pi^{n} R_{\mathcal{L}} & \rightarrow \operatorname{Hom}_{C}^{s e q}\left(T_{\mathcal{L}}, C / \pi^{n} C\right) \\
\alpha & \mapsto\left(x \mapsto \mathbb{T}_{\mathcal{L} / S}(\alpha x)\right) .
\end{aligned}
$$

Proof. Immediate from the very definition of $R_{\mathcal{L}}$. 
Let $M / L$ be a finite extension of local fields containing the local field $S$. Let $\mathcal{M}=M\left\{\left\{T_{1}\right\}\right\} \cdots\left\{\left\{T_{d-1}\right\}\right\}, \mathcal{L}=L\left\{\left\{T_{1}\right\}\right\} \cdots\left\{\left\{T_{d-1}\right\}\right\}$ and $\operatorname{Tr}_{\mathcal{M} / \mathcal{L}}$ the trace of the finite extension $\mathcal{M} / \mathcal{L}$. Then

Lemma 5.1.4. $\mathbb{T}_{\mathcal{M} / S}=\mathbb{T}_{\mathcal{L} / S} \circ \operatorname{Tr}_{\mathcal{M} / \mathcal{L}}$

Proof. By induction on $d$. Let $\mathcal{M}=\mathcal{M}^{\prime}\left\{\left\{T_{d}\right\}\right\}$ and $\mathcal{L}=\mathcal{L}^{\prime}\left\{\left\{T_{d}\right\}\right\}$, where

$$
\mathcal{M}^{\prime}=M\left\{\left\{T_{1}\right\}\right\} \cdots\left\{\left\{T_{d-1}\right\}\right\} \quad \text { and } \quad \mathcal{L}^{\prime}=L\left\{\left\{T_{1}\right\}\right\} \cdots\left\{\left\{T_{d-1}\right\}\right\} .
$$

Let $\sum_{i} a_{i} T_{d}^{i} \in \mathcal{M}$, then since $\operatorname{Tr}_{\mathcal{M} / \mathcal{L}}$ is continuous we have

$$
\operatorname{Tr}_{\mathcal{M} / \mathcal{L}}\left(\sum_{i} a_{i} T_{d}^{i}\right)=\sum_{i} \operatorname{Tr}_{\mathcal{M} / \mathcal{L}}\left(a_{i}\right) T_{d}^{i}=\sum_{i} \operatorname{Tr}_{\mathcal{M}^{\prime} / \mathcal{L}^{\prime}}\left(a_{i}\right) T_{d}^{i}
$$

Thus, since $\mathbb{T}_{\mathcal{L} / S}=\operatorname{Tr}_{L / S} \circ c_{\mathcal{L} / \mathcal{L}^{\prime}}$, then

$$
\mathbb{T}_{\mathcal{L} / S} \circ \operatorname{Tr}_{\mathcal{M} / \mathcal{L}}\left(\sum_{i} a_{i} T_{d}^{i}\right)=\mathbb{T}_{\mathcal{L}^{\prime} / S} \circ c_{\mathcal{L} / \mathcal{L}^{\prime}}\left(\sum_{i} \operatorname{Tr}_{\mathcal{M}^{\prime} / \mathcal{L}^{\prime}}\left(a_{i}\right) T_{d}^{i}\right)=\mathbb{T}_{\mathcal{L}^{\prime} / S}\left(\operatorname{Tr}_{\mathcal{M}^{\prime} / \mathcal{L}^{\prime}}\left(a_{0}\right)\right)
$$

On the other hand

$$
\mathbb{T}_{\mathcal{M} / S}\left(\sum_{i} a_{i} T_{d}^{i}\right)=\mathbb{T}_{M^{\prime} / S} \circ c_{\mathcal{M} / \mathcal{M}^{\prime}}\left(\sum_{i} a_{i} T_{d}^{i}\right)=\mathbb{T}_{\mathcal{M}^{\prime} / S}\left(a_{0}\right) .
$$

The equality follows by the induction hypothesis. 


\subsection{The map $\psi_{\mathcal{L}, n}^{i}$}

We will denote by $(,)_{\mathcal{L}, n}^{i}$ the $i$ th coordinate of the paring $(,)_{\mathcal{L}, n}$ with respect to the base $\left\{e_{i}\right\}$ of $\kappa_{n}$. Recall that a pair $(n, t)$ is said to be admissible if there exist a $k$ such that $t-1-n \geq \alpha k \geq n$, where $\alpha$ denotes the ramification index of $S$ over $\mathbb{Q}_{p}$.

Proposition 5.2.1. Let $L \supset K_{t},(n, t)$ admissible and $\mathcal{L}=L\left\{\left\{T_{1}\right\}\right\} \cdots\left\{\left\{T_{d-1}\right\}\right\}$. For a given $\alpha \in K_{d}(\mathcal{L})$ there exist a unique element $\psi_{\mathcal{L}, n}^{i}(\alpha) \in R_{\mathcal{L}, 1} / \pi^{n} R_{\mathcal{L}, 1}$, such that

$$
(\alpha, x)_{\mathcal{L}, n}^{i}=\mathbb{T}_{\mathcal{L} / S}\left(\psi_{\mathcal{L}, n}^{i}(\alpha) l_{F}(x)\right) \quad \forall x \in F\left(\mu_{\mathcal{L}, 1}\right)
$$

and the map $\psi_{\mathcal{L}, n}^{i}: K_{d}(\mathcal{L}) \rightarrow R_{\mathcal{L}, 1} / \pi^{n} R_{\mathcal{L}, 1}$ is a homomorphism.

Proof. Let us first take $\alpha$ to be an element of the form $\left\{a_{1}, \ldots, a_{d}\right\}$ and consider the map

$$
\omega: \mu_{\mathcal{L}, 1} \rightarrow C / \pi^{n} C
$$

defined by

$$
x \mapsto\left(\alpha, l_{F}^{-1}(x)\right)_{\mathcal{L}, n}^{i}
$$

By Proposition 4.2.2 and Remark 3.3.1 this map is sequentially continuous and so by Lemma 5.1 .2 there exist and element $\psi_{\mathcal{L}, n}^{i}(\alpha) \in R_{\mathcal{L}, 1} / \pi^{n} R_{\mathcal{L}, 1}$ satisfying (5.7). This defines a map $\psi_{\mathcal{L}, n}^{i}: \mathcal{L}^{* \oplus d} \rightarrow R_{\mathcal{L}, 1} / \pi^{n} R_{\mathcal{L}, 1}$ satisfying the Steinberg relation, therefore it induces a map on $K_{d}(\mathcal{L})$. 
Proposition 5.2.2. Let $M / L / K_{t}$ be a finite tower such that $(n, t)$ is admissible. Let $\mathcal{M}=M\left\{\left\{T_{1}\right\}\right\} \cdots\left\{\left\{T_{d-1}\right\}\right\}$ and $\mathcal{L}=L\left\{\left\{T_{1}\right\}\right\} \cdots\left\{\left\{T_{d-1}\right\}\right\}$. Then

1. $\operatorname{Tr}_{\mathcal{M} / \mathcal{L}}\left(R_{\mathcal{M}, 1}\right) \subset R_{\mathcal{L}, 1}$ and we have the commutative diagram

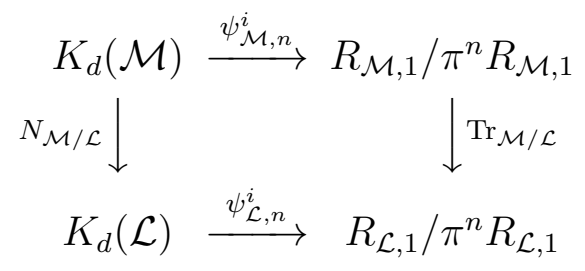

2. $R_{\mathcal{L}, 1} \subset R_{\mathcal{M}, 1}$ and we have the commutative diagram

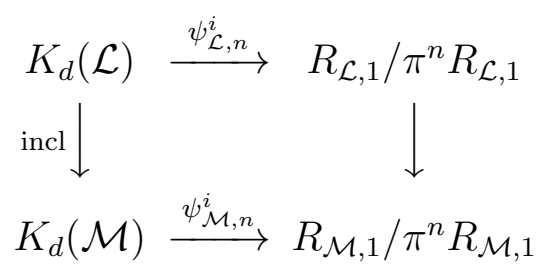

The right-hand vertical map is induced by the embedding of $R_{\mathcal{L}, 1}$ in $R_{\mathcal{M}, 1}$.

3. Let $L \supset K_{m},(m, t)$ admissible and $m \geq n$. Then for $\alpha \in K_{d}(\mathcal{L}), \psi_{\mathcal{L}, n}^{i}(\alpha)$ is the reduction of $\psi_{\mathcal{L}, m}^{i}(\alpha)$ from $R_{\mathcal{L}, 1} / \pi^{m} R_{\mathcal{L}, 1}$ to $R_{\mathcal{L}, 1} / \pi^{n} R_{\mathcal{L}, 1}$, i.e., the diagram

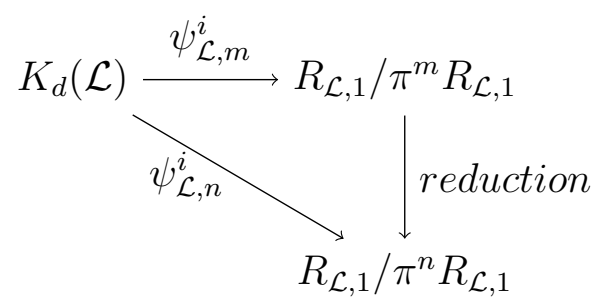

commutes. 
4. If $t:\left(F, e_{i}\right) \rightarrow\left(\tilde{F}, \tilde{e}_{i}\right)$ is an isomorphism, then

$$
\tilde{\psi}_{\mathcal{L}, n}^{i}=\frac{1}{t^{\prime}(0)} \psi_{\mathcal{L}, n}^{i}
$$

Proof. 1. By Lemma 5.1.4 and the fact that $\mu_{\mathcal{L}, 1} \subset \mu_{\mathcal{M}, 1}$ we obtain

$$
\mathbb{T}_{\mathcal{L} / S}\left(\operatorname{Tr}_{\mathcal{M} / \mathcal{L}}\left(R_{\mathcal{M}, 1}\right) \mu_{\mathcal{L}, 1}\right)=\mathbb{T}_{\mathcal{L} / S}\left(\operatorname{Tr}_{\mathcal{M} / \mathcal{L}}\left(R_{\mathcal{M}, 1} \mu_{\mathcal{L}, 1}\right)\right) \subset \mathbb{T}_{\mathcal{M} / S}\left(R_{\mathcal{M}, 1} \mu_{\mathcal{M}, 1}\right) \subset C
$$

from which follows that $\operatorname{Tr}_{\mathcal{M} / \mathcal{L}}\left(R_{\mathcal{M}, 1}\right) \subset R_{\mathcal{L}, 1}$. Now by Proposition 4.2.1 (4) we have, for $b \in K_{d}(\mathcal{M})$ and $x \in F\left(\mu_{\mathcal{L}, 1}\right)$, that

$$
\left(N_{\mathcal{M} / \mathcal{L}}(b), x\right)_{\mathcal{L}, n}=(b, x)_{\mathcal{M}, n}=\mathbb{T}_{\mathcal{M} / S}\left(\psi_{\mathcal{M}, m}^{i}(b) l(x)\right)=\mathbb{T}_{\mathcal{L} / S}\left(\operatorname{Tr}_{\mathcal{M} / \mathcal{L}}\left(\psi_{\mathcal{M}, m}^{i}(b)\right) l(x)\right)
$$

It follows from Proposition 5.2.1 that

$$
\psi_{\mathcal{L}, m}^{i}\left(N_{\mathcal{M} / \mathcal{L}}(b)\right)=\operatorname{Tr}_{\mathcal{M} / \mathcal{L}}\left(\psi_{\mathcal{M}, m}^{i}(b)\right)
$$

2. This is proved in a similar fashion to the previous property but this time using Proposition 4.2.1 (7).

3. This follows from Proposition 5.2.1 and Proposition 4.2.1 (5). Indeed, since $e_{n}^{i}=f^{(m-n)}\left(e_{m}^{i}\right)$ and $(a, x)_{n}=\left(a, f^{(m-n)}(x)\right)_{m}$ we get

$$
\pi^{m-n}(a, x)_{n}^{i}=\left(a, f^{(m-n)}(x)\right)_{m}^{i} \quad\left(\bmod \pi^{m} C\right)
$$


That is

$$
\begin{aligned}
\pi^{m-n} \mathbb{T}_{\mathcal{L} / S}\left(\psi_{\mathcal{L}, n}^{i}(a) l_{F}(x)\right) & =\mathbb{T}_{\mathcal{L} / S}\left(\psi_{\mathcal{L}, m}^{i}(a) l_{F}\left(f^{(m-n)}(x)\right)\right) \\
& =\pi^{m-n} \mathbb{T}_{\mathcal{L} / S}\left(\psi_{\mathcal{L}, m}^{i}(a) l_{F}(x)\right) \quad\left(\bmod \pi^{m} C\right)
\end{aligned}
$$

Upon dividing by $\pi^{m-n}$ the result follows.

4. This property follows from Proposition 4.2.1 $(8)$ and $l_{\tilde{F}}(t)=t^{\prime}(0) l_{F}$.

Proposition 5.2.3. Let $L / K_{t}$ be finite extension, $(n, t)$ an admissible pair, and $\mathcal{L}=L\left\{\left\{T_{1}\right\}\right\} \cdots\left\{\left\{T_{d-1}\right\}\right\}$. Let $r$ be a t-normalized series. Then

$$
\left(\left\{a_{1}, \ldots, a_{d-1}, u\right\}, x\right)_{\mathcal{L}, n}^{i}=\mathbb{T}_{\mathcal{L} / S}\left(\log (u)\left[\frac{-\psi_{L, n}^{i}\left(\left\{a_{1}, \ldots, a_{d-1}, r(x)\right\}\right) r(x) l_{F}^{\prime}(x)}{r^{\prime}(x)}\right]\right)
$$

for $a_{1}, \ldots, a_{d-1} \in \mathcal{L}^{*}, u \in V_{\mathcal{L}, 1}=1+\mu_{\mathcal{L}, 1}$ and $x \in F\left(\mu_{\mathcal{L}}\right)$.

Proof. The proof follows the proof of Proposition 4.1 of [14]. To simplify the notation in this proof we will denote the normalized valuation $v_{\mathcal{L}} / v_{\mathcal{L}}(p)$ by $v$. We will also omit the subscript $\mathcal{L}$ in the pairing notation. Observe that we may assume that $r(X)=X$ since we can go to the isomorphic formal group $\tilde{F}=r\left(F\left(r^{-1}(X), r^{-1}(Y)\right)\right)$ with torsion points $\tilde{e}=r\left(e_{i}\right)$ through the isomorphism $r: F \rightarrow \tilde{F}$. The series $\tilde{r}=X$ 
is $t$-normalized for $\tilde{F}$ and if the result were true for $\tilde{F}$ and $\tilde{r}$ then

$$
\begin{aligned}
\left(\left\{a_{1}, \ldots, a_{d-1}, u\right\}, x\right)_{F, n}^{i} & =\left(\left\{a_{1}, \ldots, a_{d-1}, u\right\}, r(x)\right)_{\tilde{F}, n}^{i} \\
& =\mathbb{T}_{\mathcal{L} / S}\left(\log (u)\left(-\tilde{\psi}_{n}^{i}\left(a_{1}, \ldots, a_{d-1}, r(x)\right) l_{\tilde{F}}^{\prime}(r(x)) r(x)\right)\right),
\end{aligned}
$$

and since $\tilde{\psi}_{n}^{i}\left(a_{1}, \ldots, a_{d-1}, r(x)\right)=r^{\prime}(0)^{-1} \psi_{n}^{i}\left(a_{1}, \ldots, a_{d-1}, r(x)\right)$ and

$$
l_{\tilde{F}}(r(X))=r^{\prime}(0) l_{F}(X) \Longrightarrow l_{\tilde{F}}^{\prime}(r(X))=r^{\prime}(0) l_{F}^{\prime}(x) / r^{\prime}(x),
$$

the result follows.

We assume therefore that $r(X)=X$. Since the pair $(n, t)$ is admissible, let $k$ be an integer such that $t-1-n \geq k \alpha \geq n, \alpha$ is the ramification index of $S / \mathbb{Q}_{p}$, and denote by $\epsilon$ the unit $\pi^{\alpha k} / p^{k}$. Let $u \in V_{\mathcal{L}, 1}=\{x \in \mathcal{L}: v(x-1)>1 /(p-1)\}$ and $x \in \mu_{\mathcal{L}}$. By bilinearity of the pairing

$$
\left(\left\{a_{1}, \ldots, a_{d-1}, u\right\}, x\right)_{n}=\left(\left\{a_{1}, \ldots, a_{d-1}, u\right\}, x \ominus\left(x \times u^{p^{k}}\right)\right)_{n} \oplus\left(\left\{a_{1}, \ldots, a_{d-1}, u\right\}, x \times u^{p^{k}}\right)_{n} .
$$


Now let $m=n+\alpha k$ and $y=x \times u^{p^{k}}$. Then by (5) of Proposition 4.2.1

$$
\begin{aligned}
\left(\left\{a_{1}, \ldots, a_{d-1}, u\right\}, y\right)_{n} & =f^{(m-n)}\left(\left(\left\{a_{1}, \ldots, a_{d-1}, u\right\}, y\right)_{m}\right) \\
& =\pi^{\alpha k}\left(\left\{a_{1}, \ldots, a_{d-1}, u\right\}, y\right)_{m} \\
& =\epsilon\left(\left\{a_{1}, \ldots, a_{d-1}, u^{p^{k}}\right\}, y\right)_{m} \\
& =\epsilon\left(\left\{a_{1}, \ldots, a_{d-1}, \frac{y}{x}\right\}, y\right)_{m} \\
& =\epsilon\left(\left\{a_{1}, \ldots, a_{d-1}, y\right\}, y\right)_{m} \ominus \epsilon\left(\left\{a_{1}, \ldots, a_{d-1}, x\right\}, y\right)_{m} .
\end{aligned}
$$

But $r=X$ is $t$-normalized, hence we may replace $\left(\left\{a_{1}, \ldots, a_{d-1}, y\right\}, y\right)_{m}=0$ by the expression $\left(\left\{a_{1}, \ldots, a_{d-1}, x\right\}, x\right)_{m}=0$ and obtain

$$
\begin{aligned}
\left(\left\{a_{1}, \ldots, a_{d-1}, u\right\}, y\right)_{n} & =\epsilon\left(\left\{a_{1}, \ldots, a_{d-1}, x\right\}, x\right)_{m} \ominus \epsilon\left(\left\{a_{1}, \ldots, a_{d-1}, x\right\}, y\right)_{m} \\
& =\epsilon\left(\left\{a_{1}, \ldots, a_{d-1}, x\right\}, x \ominus y\right)_{m} .
\end{aligned}
$$

By the properties of the logarithm in Proposition 3.3.1 we can express

$$
u^{p^{k}}=\exp \left(\log \left(u^{p^{k}}\right)\right)=\exp \left(p^{k} \log (u)\right)=1+p^{k} \log (u)+p^{2 k} w
$$

where $w=\frac{z^{2}}{2 !}+p^{k} \frac{z^{3}}{3 !}+\cdots$, with $z=\log (u)$. Since $v(z)>1 /(p-1)$ then $v\left(\frac{z^{i}}{i !}\right)>$ $1 /(p-1)$ and so $v(w)>1 /(p-1)$. This follows, for example, by Proposition 2.4 of [14.

Since $x \ominus y \equiv x-y(\bmod x y)$ and $y=x u^{p^{k}}$ with $v(x)>0$, then $v(x \ominus y)=$ $v(x-y)=v\left(x\left(u^{p^{k}}-1\right)\right)>1 /(p-1)$. Thus, using the Taylor expansion of $l=l_{F}$ 
around $X=x$ we obtain

$$
l(y)=l\left(x+x p^{k} z+x p^{2 k} w\right)=l(x)+l^{\prime}(x)\left(x p^{k} z+x p^{2 k} w\right)+p^{2 k} w_{1}
$$

where $w_{1}=l^{\prime \prime}(x) \frac{\delta^{2}}{2 !}+p^{k} l^{(3)}(x) \frac{\delta^{3}}{3 !}+\cdots$ with $\delta=x z+x p^{k} w$. Since $v(\delta)>1 /(p-1)$ then $v\left(w_{1}\right)>1 /(p-1)$. Moreover

$$
l(y)=l(x)+l^{\prime}(x) x p^{k} z+p^{2 k} w_{2},
$$

with $v\left(w_{2}\right)>1 /(p-1)$. Then

$$
l(x \ominus y)=-l^{\prime}(x) x p^{k} z-p^{2 k} w_{2} .
$$

Observing that $-l^{\prime}(x) x z-p^{k} w_{2} \in \mu_{\mathcal{L}, 1}$ we have by the isomorphism given in 3.3.1 that there is an $\eta \in F\left(\mu_{\mathcal{L}, 1}\right)$ such that $l(x \ominus y)=p^{k} l(\eta)=-l^{\prime}(x) x p^{k} z-p^{2 k} w_{2}$. Thus

$$
x \ominus y=\left[p^{k}\right](\eta)=\left[\pi^{\alpha k}\right](\tilde{\eta})=f^{(\alpha k)}(\tilde{\eta}),
$$

for $\tilde{\eta}=\left[\epsilon^{-1}\right]_{F}(\eta)$. Since $n \geq \alpha k$ then $\pi^{n}$ divides $\pi^{\alpha k}$ and we have that $x \ominus y \in$ $f^{(n)}\left(F\left(\mu_{\mathcal{L}, 1}\right)\right)$. Thus the first term on the right hand side of equation 5.9 is zero. 
By equation (5.10) and (5) of Proposition 4.2.1 we have

$$
\begin{aligned}
\left(\left\{a_{1}, \ldots, a_{d-1}, u\right\}, x\right)_{n} & =\epsilon\left(\left\{a_{1}, \ldots, a_{d-1}, x\right\}, x \ominus y\right)_{m} \\
& =\epsilon\left(\left\{a_{1}, \ldots, a_{d-1}, x\right\}, f^{(\alpha k)}(\tilde{\eta})\right)_{m} \\
& =\epsilon\left(\left\{a_{1}, \ldots, a_{d-1}, x\right\}, \tilde{\eta}\right)_{n} \\
& =\left(\left\{a_{1}, \ldots, a_{d-1}, x\right\}, \eta\right)_{n}
\end{aligned}
$$

Since $v(\eta)>1 /(p-1)$ and $(n, t)$ is admissible we can use Proposition 5.2.1.

$$
\begin{aligned}
\left(\left\{a_{1}, \ldots, a_{d-1}, x\right\}, \eta\right)_{n} & =\mathbb{T}_{\mathcal{L} / S}\left(\psi_{n}^{i}\left(a_{1}, \ldots, a_{d-1}, x\right) l_{F}(\eta)\right) \\
& =\mathbb{T}_{\mathcal{L} / S}\left(\psi_{n}^{i}\left(a_{1}, \ldots, a_{d-1}, x\right)\left(-l^{\prime}(x) x z-p^{k} w_{2}\right)\right) .
\end{aligned}
$$

Since $\psi_{L, n}^{i}\left(a_{1}, \ldots, a_{d-1}, x\right) \in R_{L, 1}, w_{2} \in T_{L, 1}, \pi^{n} \mid p^{k}$ and $\mathbb{T}_{\mathcal{L} / S}\left(R_{L, 1} T_{L, 1}\right) \subset C$, then we can write the above as

$$
\mathbb{T}_{\mathcal{L} / S}\left(\psi_{n}^{i}\left(a_{1}, \ldots, a_{d-1}, x\right)\left(-l^{\prime}(x) x z\right)\right) .
$$

From equation 5.12 we get

$$
\left(\left\{a_{1}, \ldots, a_{d-1}, u\right\}, x\right)_{n}^{i}=\mathbb{T}_{\mathcal{L} / S}\left(\psi_{n}^{i}\left(a_{1}, \ldots, a_{d-1}, x\right)\left(-l^{\prime}(x) x z\right)\right) .
$$

Keeping in mind that $z=\log (u)$, the proposition follows. 


\subsection{The map $\rho_{\mathcal{L}, n}^{i}$}

We can define a $C$-linear structure on $V_{\mathcal{L}, 1}=1+\mu_{\mathcal{L}, 1}$ by using the isomorphism $\log : V_{\mathcal{L}, 1} \rightarrow T_{\mathcal{L}, 1}$, i.e., $c u:=\log ^{-1}(c \log (u))$. Let $x \in F\left(\mu_{\mathcal{L}}\right)$ and $a_{1}, \ldots, a_{d-1} \in \mathcal{L}^{*}$ be fixed. Consider the mapping

$$
V_{\mathcal{L}, 1} \rightarrow C / \pi^{n} C
$$

defined by

$$
u \mapsto\left(\left\{a_{1}, \ldots, a_{d-1}, u\right\}, x\right)_{\mathcal{L}, n}^{i}
$$

According to Proposition 5.2 .3 this is a continuous $C$-linear map and we have the following

Proposition 5.3.1. There exist a unique element $\rho_{\mathcal{L}, n}^{i}\left(\left\{a_{1}, \ldots, a_{d-1}\right\}, x\right) \in R_{\mathcal{L}, 1} / \pi^{n} R_{\mathcal{L}, 1}$ such that

$$
\left(\left\{a_{1}, \ldots, a_{d-1}, u\right\}, x\right)_{\mathcal{L}, n}^{i}=\mathbb{T}_{\mathcal{L} / S}\left(\log (u) \rho_{\mathcal{L}, n}^{i}\left(\left\{a_{1}, \ldots, a_{d-1}\right\}, x\right)\right)
$$

for all $u \in V_{\mathcal{L}, 1}$, and the map $\rho_{\mathcal{L}, n}^{i}: K_{d-1}(\mathcal{L}) \otimes F\left(\mu_{\mathcal{L}}\right) \rightarrow R_{\mathcal{L}, 1} / \pi^{n} R_{\mathcal{L}, 1}$ is a homomorphism.

From Proposition 5.3.1 and Proposition 5.2.3 it follows the next proposition.

Proposition 5.3.2. Let $L / K_{t}$ be a finite extension, $\mathcal{L}=L\left\{\left\{T_{1}\right\}\right\} \cdots\left\{\left\{T_{d-1}\right\}\right\}$, with 
$(n, t)$ admissible, and let $r$ be a t-normalized series. Then

$$
\frac{\psi_{\mathcal{L}, n}^{i}\left(\left\{a_{1}, \ldots, a_{d-1}, r(x)\right\}\right) r(x)}{r^{\prime}(x)}=-\frac{\rho_{\mathcal{L}, n}^{i}\left(\left\{a_{1}, \ldots, a_{d-1}\right\}, x\right)}{l_{F}^{\prime}(x)} .
$$

for all $a_{1}, \ldots, a_{d-1} \in \mathcal{L}^{*}$ and all $x \in F\left(\mu_{\mathcal{L}}\right)$.

\subsection{The maps $D_{\mathcal{L}, n}^{i}$}

Assume $L \supset K\left(\kappa_{t}\right)$ for $(n, t)$ admissible and let $\mathcal{L}=L\left\{\left\{T_{1}\right\}\right\} \ldots\left\{\left\{T_{d-1}\right\}\right\}$. Define $D_{\mathcal{L}, n}^{i}: \mathcal{O}_{\mathcal{L}}^{d} \rightarrow R_{\mathcal{L}, 1} / \pi^{n} R_{\mathcal{L}, 1}$ by

$$
D_{\mathcal{L}, n}^{i}\left(a_{1}, \ldots, a_{d}\right)=\psi_{\mathcal{L}, n}^{i}\left(\left\{a_{1} \ldots, a_{d}\right\}\right) a_{1} \cdots a_{d}
$$

and consider $R_{\mathcal{L}, 1} / \pi^{n} R_{\mathcal{L}, 1}$ as an $\mathcal{O}_{\mathcal{L} \text {-module. This map satisfies }}$

Proposition 5.4.1. 1. Leibniz Rule:

$$
D_{\mathcal{L}, n}^{i}\left(a_{1}, \ldots, a_{i} a_{i}^{\prime}, \ldots, a_{d}\right)=a_{i} D_{\mathcal{L}, n}^{i}\left(a_{1}, \ldots, a_{i}^{\prime}, \ldots, a_{d}\right)+a_{i}^{\prime} D_{\mathcal{L}, n}^{i}\left(a_{1}, \ldots, a_{i}, \ldots, a_{d}\right)
$$

2. Steinberg relation:

$$
D_{\mathcal{L}, n}^{i}\left(a_{1}, \ldots, a_{i}, \ldots, 1-a_{i}, \ldots, a_{d}\right)=0
$$

3. Skew-symmetric:

$$
D_{\mathcal{L}, n}^{i}\left(a_{1}, \ldots, a_{i}, \ldots, a_{j}, \ldots, a_{d}\right)=-D_{\mathcal{L}, n}^{i}\left(a_{1}, \ldots, a_{j}, \ldots, a_{i}, \ldots, a_{d}\right) .
$$


4. $D_{\mathcal{L}, n}^{i}\left(a_{1}, \ldots, a_{i}^{p^{k}}, \ldots, a_{d}\right)=0$ if $\pi^{n} \mid p^{k}$.

Proof. Property (1) follows from the fact that $\psi_{\mathcal{L}, n}^{i}$ is a homomorphism. Property (2) follows from the Steinberg relation $\left\{a_{1}, \ldots, a_{i}, \ldots, 1-a_{i}, \ldots, a_{d}\right\}=1$ for elements in the Milnor K-group $K_{d}(\mathcal{L})$ and property (3) follows from the fact that

$$
\left\{a_{1}, \ldots, a_{i}, \ldots, a_{j}, \ldots, a_{d}\right\}=\left\{a_{1}, \ldots, a_{j}, \ldots, a_{i}, \ldots, a_{d}\right\}^{-1}
$$

in $K_{d}(\mathcal{L})$ ( cf. Proposition 4.1.1).

Proposition 5.4.2. Let $\mathcal{L}$ be as in Proposition 5.3.2, then

$$
\frac{D_{\mathcal{L}, n}^{i}\left(a_{1}, \ldots, a_{d-1}, r(x \oplus y)\right)}{r^{\prime}(x \oplus y)}=F_{X}(x, y) \frac{D_{\mathcal{L}, n}^{i}\left(a_{1}, \ldots, a_{d-1}, r(x)\right)}{r^{\prime}(x)}+F_{Y}(x, y) \frac{D_{\mathcal{L}, n}^{i}\left(a_{1}, \ldots, a_{d-1}, r(y)\right)}{r^{\prime}(y)},
$$

for all $a_{1}, \ldots, a_{d-1} \in \mathcal{L}^{*}$ and $x, y \in F\left(\mu_{\mathcal{L}}\right)$.

Proof. This follows from the fact that

$$
\rho_{\mathcal{L}, n}^{i}\left(\left\{a_{1}, \ldots, a_{d-1}\right\}, x \oplus_{F} y\right)=\rho_{\mathcal{L}, n}^{i}\left(\left\{a_{1}, \ldots, a_{d-1}\right\}, x\right)+\rho_{\mathcal{L},}^{i}\left(\left\{a_{1}, \ldots, a_{d-1}\right\}, y\right),
$$

and from differentiating $l_{F}(F(X, Y))=l_{F}(X)+l_{F}(Y)$ with respect to $X$ and $Y$.

Let $\tilde{F}$ be the formal group $r\left(F\left(r^{-1}(X), r^{-1}(Y)\right)\right)$. The series $r(X)=X$ is $t$ normalized for $\tilde{F}$. Denote by $\tilde{\oplus}$ the sum according to this formal group and $\tilde{D}_{\mathcal{L}, n}^{i}$, 
$\tilde{\psi}_{\mathcal{L}, n}^{i}$ the corresponding maps. According to Proposition 5.2.2 (4) we have that

$$
\tilde{D}_{\mathcal{L}, n}^{i}=\frac{1}{r^{\prime}(0)} D_{\mathcal{L}, n}^{i}
$$

Therefore, Proposition 5.4.2 in terms of $\tilde{\oplus}$ and $D_{\mathcal{L}, n}^{i}$ reads as

Corollary 5.4.1. Let $\mathcal{L}$ be as in Proposition 5.3.2, then

$$
D_{\mathcal{L}, n}^{i}\left(a_{1}, \ldots, a_{d-1}, x \tilde{\oplus} y\right)=\tilde{F}_{X}(x, y) D_{\mathcal{L}, n}^{i}\left(a_{1}, \ldots, a_{d-1}, x\right)+\tilde{F}_{Y}(x, y) D_{\mathcal{L}, n}^{i}\left(a_{1}, \ldots, a_{d-1}, y\right)
$$

for all $a_{1}, \ldots, a_{d-1} \in \mathcal{L}^{*}$ and $x, y \in F\left(\mu_{\mathcal{L}}\right)$.

Let $A$ be the set of all series in $X_{d} \mathcal{O}_{\mathcal{L}}\left[\left[X_{1}, \ldots, X_{d}\right]\right]$ of the form

$$
\bigoplus_{k=1}^{\infty}\left(\bigoplus_{\substack{i=\left(i_{1}, \ldots, i_{d-1}\right) \\ 0 \leq i_{1}, \ldots, i_{d-1} \leq p^{n}-1}} \gamma_{i, k}^{p^{n}} X_{1}^{i_{1}} \cdots X_{d-1}^{i_{d-1}} X_{d}^{k}\right),
$$

where $\gamma_{i, k} \in \mathcal{O}_{\mathcal{L}}$. For $d=2$, the series looks like

$$
\bigoplus_{k=1}^{\infty}\left(\bigoplus_{0 \leq i \leq p^{n}-1} \gamma_{i, k}^{p^{n}} X_{1}^{i} X_{2}^{k}\right)
$$

where $\gamma_{i, k} \in \mathcal{O}_{\mathcal{L}}$.

Proposition 5.4.3. For $x \in \mathcal{O}_{\mathcal{L}}$, there exist elements $\gamma_{i} \in \mathcal{O}_{\mathcal{L}}, i=\left(i_{1}, \ldots i_{d-1}\right)$, such that

$$
x \equiv \sum_{\substack{i=\left(i_{1}, \ldots, i_{d-1}\right) \\ 0 \leq i_{1}, \ldots, i_{d-1} \leq p^{n}-1}} \gamma_{i}^{p^{n}} T_{1}^{i_{1}} \cdots T_{d-1}^{i_{d-1}} \quad\left(\bmod \pi_{L}\right) .
$$


Proof. By induction on $d$. For $d=1$, the result follows since $k_{L}$ is perfect. Suppose it is proved for $R=\mathcal{O}_{\mathcal{L}_{d}}$, where $\mathcal{L}_{d}=L\left\{\left\{T_{1}\right\}\right\} \ldots\left\{\left\{T_{d-1}\right\}\right\}$. Let $\mathcal{L}=\mathcal{L}_{d}\left\{\left\{T_{d}\right\}\right\}$ and $x \in \mathcal{O}_{\mathcal{L}}$. Then $x=\sum_{j \geq m} a_{j} T_{d}^{j}\left(\bmod \pi_{L}\right), a_{j} \in R$. Then

$$
\begin{aligned}
x & \equiv \sum_{0 \leq i_{d}} T_{d}^{i_{d}}\left(\sum_{m \leq i_{d}+k p^{n}} a_{i_{d}+k p^{n}} T_{d}^{k p^{n}}\right) \quad\left(\bmod \pi_{L}\right) \\
& \equiv \sum_{0 \leq i_{d}} T_{d}^{i_{d}}\left(\sum_{m \leq i_{d}+k p^{n}}\left(\sum_{0 \leq i_{1}, \ldots, i_{d-1} \leq p^{n}-1} \gamma_{i_{1}, \ldots, i_{d-1}, i_{d} ; k}^{p^{n}} T_{1}^{i_{1}} \cdots T_{d-1}^{i_{d-1}}\right) T_{d}^{k p^{n}}\right) \quad\left(\bmod \pi_{L}\right) \\
& \equiv \sum_{0 \leq i_{d}} T_{1}^{i_{1}} \cdots T_{d-1}^{i_{d-1}} T_{d}^{i_{d}}\left(\sum_{k}\left(\sum_{0 \leq i_{1}, \ldots, i_{d-1} \leq p^{n}-1} \gamma_{i_{1}, \ldots, i_{d-1}, i_{d} ; k}^{p^{n}} T_{d}^{k p^{n}}\right)\right) \quad\left(\bmod \pi_{L}\right) \\
& \equiv \sum_{0 \leq i_{1}, \ldots, i_{d} \leq p^{n}-1} \gamma_{i_{1}, \ldots, i_{d}}^{p^{n}} T_{1}^{i_{1}} \cdots T_{d}^{i_{d}},
\end{aligned}
$$

where $\gamma_{i_{1}, \ldots, i_{d}}=\sum_{k} \gamma_{i_{1}, \ldots, i_{d-1}, i_{d} ; k} T_{d}^{k}$ and regrouping terms is valid since the series are absolutely convergent in the Parshin topology. Also by noticing that the congruence

$$
\sum_{c \leq k} b_{k}^{p^{n}} T^{k p^{n}} \equiv\left(\sum_{c \leq k} b_{k} T_{d}^{k}\right)^{p^{n}} \quad\left(\bmod \pi_{L}\right)
$$

holds in $k_{\mathcal{L}_{d-1}}\left(\left(T_{d}\right)\right)$, where $k_{\mathcal{L}_{d-1}}$ is the residue field of $\mathcal{L}_{d-1}$.

Remark 5.4.1. This proposition is equivalent to the following two facts:

1. $k_{L}\left(\left(T_{1}\right)\right) \ldots\left(\left(T_{d-1}\right)\right)$ is a finite extension of $k_{L}\left(\left(T_{1}^{p^{n}}\right)\right) \ldots\left(\left(T_{d-1}^{p^{n}}\right)\right)$ of degree $(d-$ 1) $p^{n}$ and generated by the elements $T_{1}^{i_{1}} \cdots T_{d-1}^{i_{d-1}}$ for $0 \leq i_{1}, \ldots, i_{d-1} \leq p^{n}-1$.

2. $k_{L}\left(\left(T_{1}^{p^{n}}\right)\right) \ldots\left(\left(T_{d-1}^{p^{n}}\right)\right)$ is the image of $k_{L}\left(\left(T_{1}\right)\right) \ldots\left(\left(T_{d-1}\right)\right)$ under the Frobenius homomorphsim

$$
\sigma_{p}: k_{L}\left(\left(T_{1}\right)\right) \ldots\left(\left(T_{d-1}\right)\right) \rightarrow k_{L}\left(\left(T_{1}\right)\right) \ldots\left(\left(T_{d-1}\right)\right)
$$


i.e., every element of $k_{L}\left(\left(T_{1}^{p^{n}}\right)\right) \ldots\left(\left(T_{d-1}^{p^{n}}\right)\right)$ has a $p^{n}$ th root in $k_{L}\left(\left(T_{1}\right)\right) \ldots\left(\left(T_{d-1}\right)\right)$.

Both facts are easily proven by induction from the fact that, for a field $k$ of characteristic $p$, the extension $\left[k((T)): k\left(\left(T^{p}\right)\right)\right]$ has degree $p$ and $\sigma_{d}(k)\left(\left(T^{p}\right)\right)$ is the image of $k((T))$ under the Frobenius homomorphism $\sigma_{p}: k((T)) \rightarrow k((T))$.

Proposition 5.4.4. Let $\mathcal{L}=L\left\{\left\{T_{1}\right\}\right\} \cdots\left\{\left\{T_{d-1}\right\}\right\}$. For $y \in \mu_{\mathcal{L}}$, there exist an $\eta \in A$ such that $y=\eta\left(T_{1}, \ldots, \pi_{L}\right)$.

Proof. Let $y \in \mu_{\mathcal{L}}$. Then by Proposition 5.4 .3

$$
\begin{aligned}
\frac{y}{\pi_{L}} & \equiv \sum_{\substack{i=\left(i_{1}, \ldots, i_{d-1}\right) \\
0 \leq i_{1}, \ldots, i_{d-1} \leq p^{n}-1}} \gamma_{i, 1}^{p^{n}} T_{1}^{i_{1}} \cdots T_{d-1}^{i_{d-1}} \quad\left(\bmod \pi_{L}\right) \\
& \equiv \frac{\bigoplus_{\substack{i=\left(i_{1}, \ldots, i_{d-1}\right) \\
0 \leq i_{1}, \ldots, i_{d-1} \leq p^{n}-1}} \gamma_{i, 1}^{p^{n}} T_{1}^{i_{1}} \cdots T_{d-1}^{i_{d-1}} \pi_{L}}{\pi_{L}}\left(\bmod \pi_{L}\right),
\end{aligned}
$$

this is,

$$
y \equiv \bigoplus_{\substack{i=\left(i_{1}, \ldots, i_{d-1}\right) \\ 0 \leq i_{1}, \ldots, i_{d-1 \leq p^{n}-1}}} \gamma_{i, 1}^{p^{n}} T_{1}^{i_{1}} \cdots T_{d-1}^{i_{d-1}} \pi_{L} \quad\left(\bmod \pi_{L}^{2}\right) .
$$

Denote $\bigoplus_{\substack{i=\left(i_{1}, \ldots, i_{d-1}\right) \\ 0 \leq i_{1}, \ldots, i_{d-1} \leq p^{n-1}}} \gamma_{i, 1}^{p^{n}} T_{1}^{i_{1}} \cdots T_{d-1}^{i_{d-1}} \pi_{L}$ by $y_{1}$. Suppose we have defined elements $y_{k}=\bigoplus_{\substack{i=\left(i_{1}, \ldots, i_{d-1}\right) \\ 0 \leq i_{1}, \ldots, i_{d-1} \leq p^{n}-1}} \gamma_{i, k}^{p^{n}} T_{1}^{i_{1}} \cdots T_{d-1}^{i_{d-1}} \pi_{L}^{k}, 1 \leq k \leq m-1$, such that

$$
y \ominus\left(\oplus_{k=1}^{m-1} y_{k}\right) \equiv 0 \quad\left(\bmod \pi_{L}^{m}\right)
$$


Then

$$
\begin{aligned}
\frac{y \ominus\left(\oplus_{k=1}^{m-1} y_{k}\right)}{\pi_{L}^{m}} & \equiv \sum_{\substack{i=\left(i_{1}, \ldots, i_{d-1}\right) \\
0 \leq i_{1}, \ldots, i_{d-1} \leq p^{n}-1}} \gamma_{i, m}^{p^{n}} T_{1}^{i_{1}} \cdots T_{d-1}^{i_{d-1}} \quad\left(\bmod \pi_{L}\right) \\
& \equiv \frac{\bigoplus_{\substack{i=\left(i_{1}, \ldots, i_{d-1}\right) \\
0 \leq i_{1}, \ldots, i_{d-1 \leq p^{n}-1}}} \gamma_{i, m}^{p^{n}} T_{1}^{i_{1}} \cdots T_{d-1}^{i_{d-1}} \pi_{L}^{m}}{\pi_{L}^{m}} \quad\left(\bmod \pi_{L}\right) .
\end{aligned}
$$

Denote $\bigoplus_{\substack{i=\left(i_{1}, \ldots, i_{d-1}\right) \\ 0 \leq i_{1}, \ldots, i_{d-1 \leq p^{n}-1}}} \gamma_{i, m}^{p^{n}} T_{1}^{i_{1}} \cdots T_{d-1}^{i_{d-1}} \pi_{L}^{m}$ by $y_{m}$. Then

$$
y \ominus\left(\oplus_{k=1}^{m-1} y_{k}\right) \equiv y_{m} \quad\left(\bmod \pi_{L}^{m+1}\right)
$$

or

$$
y \ominus\left(\oplus_{k=1}^{m-1} y_{k}\right) \equiv 0 \quad\left(\bmod \pi_{L}^{m+1}\right)
$$

Therefore $y=\oplus_{k=1}^{\infty} y_{k}$, which is what we wanted to prove.

Corollary 5.4.2. For every $x \in \mathcal{O}_{\mathcal{L}}$, there exists $\gamma_{i, k} \in \mathcal{O}_{\mathcal{L}}$ such that

$$
\sum_{k=0}^{\infty}\left(\sum_{\substack{i=\left(i_{1}, \ldots, i_{d-1}\right) \\ 0 \leq i_{1}, \ldots, i_{d-1} \leq p^{n}-1}} \gamma_{i, k}^{p^{n}} T_{1}^{i_{1}} \cdots T_{d-1}^{i_{d-1}} \pi_{L}^{k}\right)
$$

Proof. Take $F$ to be the additive formal group $X+Y$ in Proposition 5.4.4.

Corollary 5.4.3. For every $x \in V_{\mathcal{L}}=1+\mu_{\mathcal{L}}$, there exist $\gamma_{i, k} \in \mathcal{O}_{\mathcal{L}}$ such that

$$
\prod_{k=1}^{\infty}\left(\prod_{\substack{i=\left(i_{1}, \ldots, i_{d-1}\right) \\ 0 \leq i_{1}, \ldots, i_{d-1} \leq p^{n}-1}}\left(1+\gamma_{i, k}^{p^{n}} T_{1}^{i_{1}} \cdots T_{d-1}^{i_{d-1}} \pi_{L}^{k}\right)\right)
$$

Proof. This can be proven either by using Proposition 5.4.3 and induction on $k$; just as in the proof of Proposition 5.4.4, or by applying Proposition 5.4.4 to the 
multiplicative formal group $F(X, Y)=X+Y+X Y$. Noticing also, that the homomorphism

$$
F\left(\mu_{\mathcal{L}}\right) \longrightarrow V_{\mathcal{L}}
$$

defined by $x \mapsto 1+x$, is continuous in the topology induced by the valuation $v_{\mathcal{L}}$ of $\mathcal{L}$.

Let $r$ be a $t$-normalized series for $F$ and $(n, t)$ an admissible pair. Let $\tilde{F}$ be the formal group $r\left(F\left(r^{-1}(X), r^{-1}(Y)\right)\right)$. Let $\mathcal{L}=L\left\{\left\{T_{1}\right\}\right\} \ldots\left\{\left\{T_{d-1}\right\}\right\}$. For $y \in \mu_{\mathcal{L}}$ we will denote by $\tilde{\eta}_{y}\left(X_{1}, \ldots, X_{d}\right)$ the multivariable series

$$
\tilde{\oplus}_{k=1}^{\infty}\left(\tilde{\oplus}_{\substack{i=\left(i_{1}, \ldots, i_{d-1}\right) \\ 0 \leq i_{1}, \ldots, i_{d-1} \leq p^{n}-1}} \gamma_{i, k}^{p^{n}} X_{1}^{i_{1}} \cdots X_{d-1}^{i_{d-1}} X_{d}^{k}\right), \quad\left(\gamma_{i, k} \in \mathcal{O}_{\mathcal{L}}\right)
$$

with respect to $\tilde{F}$, such that $y=\tilde{\eta}_{y}\left(T_{1}, \ldots, \pi_{L}\right)$, whose existence is guaranteed by Proposition 5.4.4.

Proposition 5.4.5. Let $L / K_{t}$ be a finite extension with $(n, t)$ admissible and set $\mathcal{L}=L\left\{\left\{T_{1}\right\}\right\} \ldots\left\{\left\{T_{d-1}\right\}\right\}$. For $y=\tilde{\eta}_{y}\left(T_{1}, \ldots, \pi_{L}\right) \in \mu_{\mathcal{L}}, \tilde{\eta}_{y}$ as in (5.22), we have

$$
D_{\mathcal{L}, n}^{i}\left(a_{1}, \ldots, a_{d-1}, y\right)=\left.\sum_{i=1}^{d} \frac{\partial \tilde{\eta}_{y}}{\partial X_{i}}\right|_{\substack{X_{k}=T_{k}, k=1, \ldots, d}} D_{\mathcal{L}, n}^{i}\left(a_{1}, \ldots, a_{d-1}, T_{i}\right),
$$

for all $a_{1}, \ldots, a_{d-1} \in \mathcal{O}_{\mathcal{L}}$, where $T_{d}=\pi_{L}$.

Proof. Let $y=\oplus_{k=1}^{\infty} y_{k}$, where

$$
y_{k}=\tilde{\bigoplus}_{\substack{i=\left(i_{1}, \ldots, i_{d-1}\right) \\ 0 \leq i_{1}, \ldots, i_{d-1 \leq p^{n}-1}}} \gamma_{i, k}^{p^{n}} T_{1}^{i_{1}} \cdots T_{d-1}^{i_{d-1}} \pi_{L}^{k}
$$


Thus, $\tilde{\eta}_{y}=\tilde{\oplus}_{m=1}^{\infty} \eta_{m}$, where

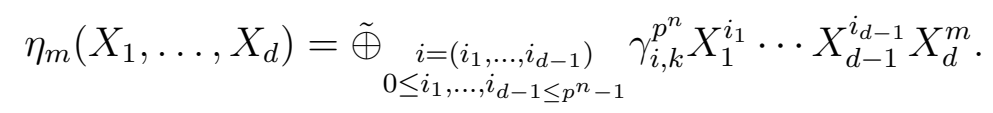

Let us fix $a_{1}, \ldots, a_{d-1} \in \mathcal{L}^{*}$ and denote by $D(x)$ the element $D_{\mathcal{L}, n}^{i}\left(a_{1}, \ldots, a_{d-1}, x\right)$ to simplify notation.

First notice that

$$
v_{\mathcal{L}}\left(y_{k}\right) \stackrel{k \rightarrow \infty}{\longrightarrow} \infty \quad \Longrightarrow \quad D\left(y_{k}\right)=0 \quad \text { for } k \text { large enough. }
$$

This follows from $D_{\mathcal{L}, n}^{i}\left(a_{1}, \ldots, a_{d-1}, x\right)=\psi_{\mathcal{L}, n}^{i}\left(a_{1}, \ldots, a_{d-1}, x\right) a_{1} \cdots a_{d-1} x$ and that $\psi_{\mathcal{L}, n}^{i}$ has values in $R_{\mathcal{L}} / \pi^{n} R_{\mathcal{L}, n} ; \pi_{L}^{n} \psi_{\mathcal{L}, n}^{i}=0$.

Thus, from $\tilde{\eta}_{y}=\tilde{\oplus}_{m=1}^{k-1} \eta_{m}\left(\bmod \pi^{k}\right)$ and equation 5.23 , it is enough to consider the finite formal sum $\tilde{\oplus}_{m=1}^{k-1} \eta_{m}$. The proposition follows now from the fact that $D\left(\gamma^{p^{n}}\right)=p^{n} \gamma^{p^{n}-1} D(\gamma)=0\left(\pi^{n} \mid p^{n}\right), D(x y)=y D(x)+x D(y)$, and corollary 5.4.1.

Corollary 5.4.4. Let $L / K_{t}$ be a finite extension with $(n, t)$ admissible and set $\mathcal{L}=$ $L\left\{\left\{T_{1}\right\}\right\} \ldots\left\{\left\{T_{d-1}\right\}\right\}$. Let $y_{i}=\tilde{\eta}_{y_{i}}\left(T_{1} \ldots, \pi_{L}\right) \in \mu_{\mathcal{L}}$, for $1 \leq i \leq d$, where $\eta_{y_{i}}$ is a multivariable series of the form 5.21). Then

$$
D_{\mathcal{L}, n}\left(y_{1}, \ldots, y_{d}\right)=\operatorname{det}\left[\frac{\partial \tilde{\eta}_{y_{i}}}{\partial X_{j}}\right]_{\substack { i, j \\
\begin{subarray}{c}{X_{k}=T_{k}, k=1, \ldots, d{ i , j \\
\begin{subarray} { c } { X _ { k } = T _ { k } , \\
k = 1 , \ldots , d } }\end{subarray}} D_{\mathcal{L}, n}\left(T_{1}, \ldots, \pi_{L}\right),
$$

where $T_{d}=\pi_{L}$. 
Proof. This follows from Proposition 5.4.5 and Proposition 5.4.1 (3). Indeed, let us illustrate the proof in the case $d=2$, i.e., $\left.\mathcal{L}=L\left\{T_{1}\right\}\right\}$ and $T_{2}=\pi_{L}$. To simplify the notation we will denote $D_{\mathcal{L}, n}^{i}$ by $D$. From Proposition 5.4.5 we have

$$
\begin{aligned}
D\left(\eta_{1}\left(T_{1}, T_{2}\right), \eta_{2}\left(T_{1}, T_{2}\right)\right)= & \left.\frac{\partial \eta_{1}}{\partial X_{1}} \frac{\partial \eta_{2}}{\partial X_{1}}\right|_{\substack{X_{i}=T_{i}, i=1,2}} D\left(T_{1}, T_{1}\right)+\left.\frac{\partial \eta_{1}}{\partial X_{1}} \frac{\partial \eta_{2}}{\partial X_{2}}\right|_{\substack{X_{i}=T_{i}, i=1,2}} D\left(T_{1}, T_{2}\right) \\
& +\left.\frac{\partial \eta_{1}}{\partial X_{2}} \frac{\partial \eta_{2}}{\partial X_{1}}\right|_{\substack{X_{i}=T_{i}, i=1,2}} D\left(T_{2}, T_{1}\right)+\left.\frac{\partial \eta_{1}}{\partial X_{2}} \frac{\partial \eta_{2}}{\partial X_{2}}\right|_{\substack{X_{i}=T_{i}, i=1,2}} D\left(T_{2}, T_{2}\right)
\end{aligned}
$$

But $D\left(T_{1}, T_{1}\right)=D\left(T_{2}, T_{2}\right)=0, D\left(T_{2}, T_{1}\right)=-D\left(T_{1}, T_{2}\right)$ from Proposition 5.4.1 (3), therefore

$$
\left.D\left(\eta_{1}\left(T_{1}, T_{2}\right), \eta_{2}\left(T_{1}, T_{2}\right)\right)=\left.\left(\frac{\partial \eta_{1}}{\partial X_{1}} \frac{\partial \eta_{2}}{\partial X_{2}}-\frac{\partial \eta_{1}}{\partial X_{2}} \frac{\partial \eta_{2}}{\partial X_{1}}\right)\right|_{\substack{x_{i}=T_{i}, i=1,2}} D\left(T_{2}, T_{1}\right)\right)
$$

The corollary follows. 


\section{Chapter 6}

\section{Multidimensional derivations}

Let $\mathcal{L}$ be the standard $d$-dimensional local field $L\left\{\left\{T_{1}\right\}\right\} \ldots\left\{\left\{T_{d-1}\right\}\right\}$ and $L / K / \mathbb{Q}_{p}$ be a tower of finite extensions. Let $W$ be an $\mathcal{O}_{\mathcal{L}}$-module that is $p$-adically complete, i.e,

$$
W \cong \lim _{\longleftarrow} W / p^{n} W .
$$

For example, if $p^{n} W=0$ for some $n$, then $W$ is $p$-adically complete. Actually, this is going to be our situation, since $W$ will be the $\mathcal{O}_{\mathcal{L}}$-module $R_{\mathcal{L}, 1} / \pi^{n} R_{\mathcal{L}, 1}$.

\subsection{Derivations and the module of differentials}

Definition 6.1.1. A derivation of $\mathcal{O}_{\mathcal{L}}$ into $W$ over $\mathcal{O}_{K}$ is a map $D: \mathcal{O}_{\mathcal{L}} \rightarrow W$ such that for all $a, b \in \mathcal{O}_{\mathcal{L}}$ we have

1. $D(a b)=a D(b)+b D(a)$.

2. $D(a+b)=D(a)+D(b)$. 
3. $D(a)=0$ if $a \in \mathcal{O}_{K}$.

We denote by $D_{\mathcal{O}_{K}}\left(\mathcal{O}_{\mathcal{L}}, W\right)$ the $\mathcal{O}_{\mathcal{L}}$-module of all derivations $D: \mathcal{O}_{\mathcal{L}} \rightarrow W$. The universal object in the category of derivations of $\mathcal{O}_{\mathcal{L}}$ over $\mathcal{O}_{K}$ is the $\mathcal{O}_{\mathcal{L}}$-module of Khaler differentials, denoted by $\Omega_{\mathcal{O}_{K}}\left(\mathcal{O}_{\mathcal{L}}\right)$. This is the $\mathcal{O}_{\mathcal{L}}$-module generated by finite linear combinations of the symbols $d a$, for all $a \in \mathcal{O}_{\mathcal{L}}$, divided out by the submodule generated by all the expressions of the form $d a b-a d b-b d a$ and $d(a+b)-d a-d b$ for all $a, b \in \mathcal{O}_{\mathcal{L}}$ and $d a$ for all $a \in \mathcal{O}_{K}$. The derivation $d: \mathcal{O}_{\mathcal{L}} \rightarrow \Omega_{\mathcal{O}_{K}}\left(\mathcal{O}_{\mathcal{L}}\right)$ is defined by sending $a$ to $d a$.

If $D: \mathcal{O}_{\mathcal{L}} \rightarrow W$ is a derivation, then $\Omega_{\mathcal{O}_{K}}\left(\mathcal{O}_{\mathcal{L}}\right)$ is universal in the following way. There exist a unique homomorphism $\beta: \Omega_{\mathcal{O}_{K}}\left(\mathcal{O}_{\mathcal{L}}\right) \rightarrow W$ of $\mathcal{O}_{\mathcal{L}}$-modules such that the diagram

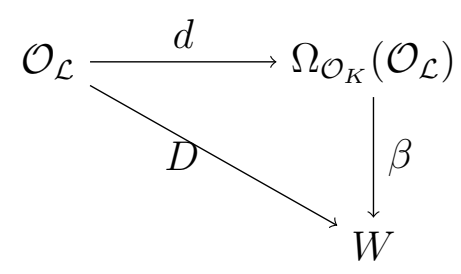

is commutative.

Let $\hat{\Omega}_{\mathcal{O}_{K}}\left(\mathcal{O}_{\mathcal{L}}\right)$ be the $p$-adic completion of $\Omega_{\mathcal{O}_{K}}\left(\mathcal{O}_{\mathcal{L}}\right)$, i.e.,

$$
\hat{\Omega}_{\mathcal{O}_{K}}\left(\mathcal{O}_{\mathcal{L}}\right)=\lim _{\longleftarrow} \Omega_{\mathcal{O}_{K}}\left(\mathcal{O}_{\mathcal{L}}\right) / p^{n} \Omega_{\mathcal{O}_{K}}\left(\mathcal{O}_{\mathcal{L}}\right)
$$

Since we are assuming that $W$ is $p$-adically complete, the homomorphism $\beta$ induces 
the homomorphism

$$
\beta: \hat{\Omega}_{\mathcal{O}_{K}}\left(\mathcal{O}_{\mathcal{L}}\right) \rightarrow W
$$

Proposition 6.1.1. We have the isomorphism of $\mathcal{O}_{\mathcal{L}}$-modules

$$
\hat{\Omega}_{\mathcal{O}_{K}}\left(\mathcal{O}_{\mathcal{L}}\right) \cong \mathcal{O}_{\mathcal{L}} d T_{1} \oplus \cdots \oplus \mathcal{O}_{\mathcal{L}} d T_{d-1} \oplus\left(\mathcal{O}_{\mathcal{L}} / D(L / K) \mathcal{O}_{\mathcal{L}}\right) d \pi_{L}
$$

where $\pi_{L}$ is a uniformizer for $L$ and $D(L / K)$ is the different of the extension $L / K$. That is, $d T_{1}, \ldots, d T_{d-1}$ and $d \pi_{L}$ generate $\hat{\Omega}_{\mathcal{O}_{K}}\left(\mathcal{O}_{\mathcal{L}}\right)$.

Proof. To simplify the notation let us denote $\hat{\Omega}_{\mathcal{O}_{K}}\left(\mathcal{O}_{\mathcal{L}}\right)$ by $\hat{\Omega}$, where $\Omega=\Omega_{\mathcal{O}_{K}}\left(\mathcal{O}_{\mathcal{L}}\right)$. We will show that

$$
\frac{\Omega}{\pi_{L}^{n} \Omega} \simeq \frac{\mathcal{O}_{\mathcal{L}}}{\pi^{n} \mathcal{O}_{\mathcal{L}}} \oplus \cdots \oplus \frac{\mathcal{O}_{\mathcal{L}}}{\pi^{n} \mathcal{O}_{\mathcal{L}}} \oplus \frac{\mathcal{O}_{\mathcal{L}}}{\pi^{n} \mathcal{O}_{\mathcal{L}}+D(L / K) \mathcal{O}_{\mathcal{L}}}
$$

for all $n \geq 1$. These isomorphisms are compatible: $\simeq_{n+1} \equiv_{n}\left(\bmod p^{n}\right)$, then we can take the projective limit $\varliminf_{\longleftarrow}$ to obtain the result. To prove 6.1 , we will start by showing that $\Omega / \pi_{L}^{n} \Omega$ is generated by $d \pi_{L}$ and $d T_{1}, \ldots, d T_{d-1}$ for all $n$.

Let $x \in \mathcal{O}_{\mathcal{L}}$, then by corollary 5.4 .2 , we have that

$$
x=\sum_{k=0}^{\infty}\left(\sum_{0 \leq i_{1}, \ldots, i_{d-1} \leq p^{n}-1} \gamma_{i, k}^{p^{n}} T_{1}^{i_{1}} \cdots T_{i_{d-1}} \pi_{L}^{k}\right) .
$$

Therefore, in $\Omega / p^{n} \Omega$, we can consider the truncated sum

$$
\sum_{k=0}^{m}\left(\sum_{0 \leq i_{1}, \ldots, i_{d-1} \leq p^{n}-1} \gamma_{i, k}^{p^{n}} T^{i} Y^{k}\right)
$$


where $m$ is such that $p^{n} \mid \pi^{m+1}$. Thus, $d x$ is generated by $d \pi_{L}$ and $d T_{i}, i=1, \ldots, d-1$ in $\Omega / p^{n} \Omega$.

Let us construct now the isomorphism 6.1). We will define derivations $D_{k}$ of $\mathcal{O}_{\mathcal{L}}$ over $\mathcal{O}_{K}$ for $k=0, \ldots, d-1$ as follows. Let $\mathcal{L}_{0}=L$ and $\mathcal{L}_{k}=\mathcal{L}_{k-1}\left\{\left\{T_{k}\right\}\right\}$, $k=1, \ldots, d-1$. For $k=0$ define

$$
D_{0}: \mathcal{O}_{L} \rightarrow \mathcal{O}_{L} / D(L / K) \mathcal{O}_{L}
$$

by $D_{0}\left(g\left(\pi_{L}\right)\right)=g^{\prime}\left(\pi_{L}\right)$, where $g(X)$ is a polynomial with coefficients in the ring of integers of the maximal subextension of $L$ unramified over $K$. This is a well defined derivation of $\mathcal{O}_{L}$ over $\mathcal{O}_{K}$ by Corollary 5.2 of [14]. For $1 \leq k \leq d-1$, we define the derivation of $\mathcal{O}_{\mathcal{L}_{k}}$ over $\mathcal{O}_{K}$

$$
D_{k}: \mathcal{O}_{\mathcal{L}_{k}} \rightarrow \mathcal{O}_{\mathcal{L}_{k}},
$$

by $D_{k}\left(\sum a_{i} T_{k}^{i}\right)=\sum a_{i} i T_{k}^{i-1}, a_{i} \in \mathcal{O}_{\mathcal{L}_{k-1}}$.

We now lift these derivations to derivations of $\mathcal{O}_{\mathcal{L}}$ over $\mathcal{O}_{K}$, by induction, in the following way. Suppose $D: \mathcal{O}_{\mathcal{L}_{k}} \rightarrow W$ is a derivation of $\mathcal{O}_{\mathcal{L}_{k-1}}$ over $\mathcal{O}_{K}$, where $1 \leq k \leq d$ and the $\mathcal{O}_{\mathcal{L}_{k}}$-module $W$ is either $\mathcal{O}_{\mathcal{L}_{k-1}}$ or $\mathcal{O}_{\mathcal{L}_{k-1}} / D(L / K) \mathcal{O}_{\mathcal{L}_{k-1}}$. Then $D$ extends to a derivation of $\mathcal{O}_{\mathcal{L}_{k}}$ over $\mathcal{O}_{K}$

$$
D: \mathcal{O}_{\mathcal{L}_{k}} \rightarrow \mathcal{O}_{\mathcal{L}_{k}} \otimes W
$$


by

$$
D\left(\sum_{i} a_{i} T_{k}^{i}\right):=\sum_{i} D\left(a_{i}\right) T_{k}^{i} .
$$

This derivation is well defined since $D$ is continuous with respect to the valuation topology of $\mathcal{O}_{\mathcal{L}_{k-1}}$.

Let us now define the map

$$
\partial: \mathcal{O}_{\mathcal{L}} \rightarrow \frac{\mathcal{O}_{\mathcal{L}}}{p^{n} \mathcal{O}_{\mathcal{L}}+\mathcal{D}(L / K) \mathcal{O}_{\mathcal{L}}} \oplus \frac{\mathcal{O}_{\mathcal{L}}}{p^{n} \mathcal{O}_{\mathcal{L}}} \oplus \cdots \oplus \frac{\mathcal{O}_{\mathcal{L}}}{p^{n} \mathcal{O}_{\mathcal{L}}}
$$

by

$$
a \rightarrow\left(\overline{D_{0}}(a), \ldots, \overline{D_{d-1}}(a)\right)
$$

where $\overline{D_{k}}$ is the reduction of $D_{k}$. This is a well-defined derivation of $\mathcal{O}_{\mathcal{L}}$ over $\mathcal{O}_{K}$ and by the universality of $\Omega$, this induces a homomorphism of $\mathcal{O}_{\mathcal{L}}$-modules

$$
\partial: \frac{\Omega}{p^{n} \Omega} \rightarrow \frac{\mathcal{O}_{\mathcal{L}}}{p^{n} \mathcal{O}_{\mathcal{L}}+D(L / K) \mathcal{O}_{\mathcal{L}}} \oplus \cdots \oplus \frac{\mathcal{O}_{\mathcal{L}}}{p^{n} \mathcal{O}_{\mathcal{L}}} .
$$

Let us show that $\partial$ is an isomorphism. Indeed, it is clearly surjective since for $\left(a_{0}, \ldots, a_{d-1}\right) \in \mathcal{O}_{\mathcal{L}} \oplus \cdots \oplus \mathcal{O}_{\mathcal{L}} \oplus\left(\mathcal{O}_{\mathcal{L}} / \mathcal{D}(L / K) \mathcal{O}_{\mathcal{L}}\right)$ we have that

$$
\partial\left(a_{0} d \pi_{L}+a_{1} d T_{1}+\cdots a_{d-1} d T_{d-1}\right)=\left(a_{0}, \ldots, a_{d-1}\right) .
$$

since

$$
\overline{D_{k}}\left(d \pi_{L}\right)=\left\{\begin{array}{ll}
0, & k=0, \\
1, & 1 \leq k \leq d-1,
\end{array} \quad \overline{D_{k}}\left(d T_{i}\right)= \begin{cases}0, & k \neq i, \\
1, & k=i,\end{cases}\right.
$$

Also, $\partial$ is injective for if $a=a_{0} d \pi_{L}+a_{1} d T_{1}+\cdots a_{d-1} d T_{d-1} \in \Omega / p^{n} \Omega$ is such that 
$\partial(a)=0$, then $\overline{D_{k}}(a)=a_{k}=0$ in $\mathcal{O}_{\mathcal{L}} / p^{n} \mathcal{O}_{\mathcal{L}}$, for $1 \leq k \leq d-1$, and $\overline{D_{0}}(a)=a_{0}=0$ $\left(\bmod p^{n} \mathcal{O}_{\mathcal{L}}+\mathcal{D}(L / K) \mathcal{O}_{\mathcal{L}}\right)$. But then $a_{0} d \pi_{L}=0$, since $\mathcal{D}(L / K) d \pi_{L}=0$, and therefore $a=0 \bmod p^{n} \Omega$. This concludes the proof.

Definition 6.1.2. The derivations $D_{k}: \mathcal{O}_{\mathcal{L}} \rightarrow \mathcal{O}_{\mathcal{L}}, 1 \leq k \leq d-1$, which are obtained by the lifts of the derivations given in equation (6.2) described in Proposition 6.1.1. will be denoted by $\frac{\partial}{\partial T_{k}}$ for $1 \leq k \leq d-1$. Moreover, the derivation $D_{0}: \mathcal{O}_{\mathcal{L}} \rightarrow$ $\mathcal{O}_{\mathcal{L}} / D(L / K) \mathcal{O}_{\mathcal{L}}$, also defined in the above proposition, will be denoted by $\frac{\partial}{\partial T_{d}}$.

Remark 6.1.1. The derivations $\frac{\partial}{\partial T_{k}}$ for $1 \leq k \leq d-1$ coincide, informally, with the partial derivative with respect to $T_{k}$ of an element $\alpha$ in $\mathcal{O}_{\mathcal{L}}=\mathcal{O}_{L}\left\{\left\{T_{1}\right\}\right\} \cdots\left\{\left\{T_{d-1}\right\}\right\}$. On the other hand, the derivation $\frac{\partial}{\partial T_{d}}$ has the following interpretation. If $\alpha \in \mathcal{O}_{\mathcal{L}}$, then there exist a polynomial $g(X) \in \mathcal{O}_{\tilde{\mathcal{L}}}[X]$ such that $\alpha=g\left(\pi_{L}\right)$. Then

$$
\frac{\partial \alpha}{\partial T_{d}}=g^{\prime}\left(\pi_{L}\right)
$$

Here $\tilde{\mathcal{L}}=\tilde{L}\left\{\left\{T_{1}\right\}\right\} \cdots\left\{\left\{T_{d-1}\right\}\right\}$, where $\tilde{L}$ is the maximal subextension of $L$ unramified over $K$.

Proposition 6.1.2. Let $D: \mathcal{O}_{\mathcal{L}} \rightarrow W$ be a derivation and let $\frac{\partial}{\partial T_{k}}, 1 \leq k \leq d$, be the derivations form Definition 6.1.2. Then $D(L / K) D\left(\pi_{\mathcal{L}}\right)=0$ and

$$
D(\beta)=\sum_{k=1}^{d-1} \frac{\partial \beta}{\partial T_{k}} D\left(T_{k}\right)+\frac{\partial \beta}{\partial T_{d}} D\left(\pi_{L}\right) .
$$


Moreover, given $w_{1}, \ldots, w_{d} \in W$ such that $D(L / K) w_{d}=0$, the map

$$
D(\beta):=\sum_{k=1}^{d} \frac{\partial \beta}{\partial T_{k}} w_{k}
$$

is a well-defined derivation from $\mathcal{O}_{\mathcal{L}}$ into $W$ over $\mathcal{O}_{K}$. In other words, the map

$$
D \mapsto\left(D\left(T_{1}\right), \ldots, D\left(\pi_{L}\right)\right)
$$

defines an isomorphism

$$
D_{\mathcal{O}_{K}}\left(\mathcal{O}_{\mathcal{L}}, W\right) \stackrel{\sim}{\rightarrow} \underbrace{W \oplus \cdots \oplus W}_{(d-1) \text {-times }} \oplus W_{D(L / K)},
$$

where $W_{\mathcal{D}(L / K)}$ is the submodule of elements killed by $D(L / K)$.

Proof. The proof follows from the proof of Proposition 6.1.1 and the fact that $D_{\mathcal{O}_{K}}\left(\mathcal{O}_{\mathcal{L}}, W\right) \cong \operatorname{Hom}_{\mathcal{O}_{\mathcal{L}}}\left(\hat{\Omega}_{\mathcal{O}_{K}}\left(\mathcal{O}_{\mathcal{L}}\right), W\right)$.

If $L$ is a finite extension of the local field $K$, then we denote by $\Omega_{\mathcal{O}_{K}}\left(\mathcal{O}_{L}\right) \cong$ $\mathcal{O}_{L} / D(L / K)$ the $\mathcal{O}_{K}$-module of differentials of $\mathcal{O}_{L}$ over $\mathcal{O}_{K}$.

Proposition 6.1.3. 1. $\Omega_{\mathcal{O}_{K}}\left(\mathcal{O}_{L}\right) \cong \mathcal{O}_{L} / D(L / K)$ as $\mathcal{O}_{L}$-modules. Moreover, the element $d \pi_{L}$ generates $\Omega_{\mathcal{O}_{K}}\left(\mathcal{O}_{L}\right)$.

2. If $M$ is a finite extension of $L$, the homomorphism $\Omega_{\mathcal{O}_{K}}\left(\mathcal{O}_{L}\right) \rightarrow \Omega_{\mathcal{O}_{K}}\left(\mathcal{O}_{M}\right)$ is an embedding. 
Proof. cf. [14] Proposition 5.1.

We will denote by $K_{m}$ (resp. $L_{m}$ ) the field obtained by adjoining the $m$-th torsion points to $K$, i.e., $K_{m}=K\left(\kappa_{m}\right)$. Let $v$ denote the normalized valuation $v_{M} / v_{M}(p)$, for every finite extension $M$ of $\mathbb{Q}_{p}$.

Proposition 6.1.4. There are positive constants $c_{1}, c_{2} \in \mathbb{R}$, depending on $(F, \pi)$, such that

1. $v\left(D\left(L_{m} / L\right)\right) \leq m / \alpha+\log _{2}(m) /(p-1)+c_{2}$ and $v\left(D\left(K_{m} / K\right)\right) \geq m / \alpha-c_{1}$. Where $\alpha$ is the ramification index of $S$ over $\mathbb{Q}_{p}$.

2. Let $1 \leq j \leq h$ be fixed. Let $p_{m}$ be the period (i.e., the generator of the annihalator ideal) of the $\mathcal{O}_{K_{m}}$-submodule of $\Omega_{\mathcal{O}_{K}}\left(\mathcal{O}_{K_{m}}\right)$ generated by de ${ }_{m}^{j}$. Then there exists a $j$ for which $v\left(p_{m}\right) \geq m / \alpha-c_{1}$.

Proof. cf. [14] Proposition 5.3.

Proposition 6.1.5. Suppose $K / S$ is an unramified extension and let $q=\left|k_{S}\right|$. Let $h$ be the height of $F$ with respect to $C=\mathcal{O}_{S}$, cf. Proposition 3.2.2. Then

1. $v\left(D\left(K_{m} / K\right)\right) \geq m / \alpha-1 / \alpha\left(q^{h}-1\right)$.

2. $K_{m}=K\left(e_{m}^{i}\right)$ is totally unramified over $K$ and $e_{m}^{i}$ is a uniformizer for $K_{m}$ and $v\left(\right.$ period of $\left.d e_{m}^{i}\right)=m / \alpha-1 / \alpha\left(q^{h}-1\right)$. 
Proof. cf. [14] Proposition 5.6.

\subsection{Multidimensional derivations}

Let $\mathcal{L}, \tilde{\mathcal{L}}, L, \tilde{L} K$ and $W$ be as in the beginning of the previous section.

Definition 6.2.1. A d dimensional derivation of $\mathcal{O}_{L}^{d}$ into $W$ over $\mathcal{O}_{K}$ is map $D$ : $\mathcal{O}_{\mathcal{L}}^{d} \rightarrow W$ such that for all $a_{1} \ldots, a_{d}$ and all $a_{1}^{\prime}, \ldots, a_{d}^{\prime}$ in $\mathcal{O}_{\mathcal{L}}$ it satisfies

1. Leibniz rule:

$$
D\left(a_{1}, \ldots, a_{i} a_{i}^{\prime}, \ldots, a_{d}\right)=a_{i}^{\prime} D\left(a_{1}, \ldots, a_{i}, \ldots, a_{d}\right)+a_{i} D\left(a_{1}, \ldots, a_{i}^{\prime}, \ldots, a_{d}\right)
$$

for any $1 \leq i \leq d$.

2. Linearity:

$$
D\left(a_{1}, \ldots, a_{i}+a_{i}^{\prime}, \ldots, a_{d}\right)=D\left(a_{1}, \ldots, a_{i}, \ldots, a_{d}\right)+D\left(a_{1}, \ldots, a_{i}^{\prime}, \ldots, a_{d}\right),
$$

for any $1 \leq i \leq d$.

3. Alternate: $D\left(a_{1}, \ldots, a_{i}, \ldots, a_{j}, \ldots, a_{d}\right)=0$, if $a_{i}=a_{j}$ for $i \neq j$.

4. $D\left(a_{1}, \ldots, a_{d}\right)=0$ if some $a_{i} \in \mathcal{O}_{K}$.

We denote by $D_{\mathcal{O}_{\mathcal{K}}}^{d}\left(\mathcal{O}_{\mathcal{L}}^{d}, W\right)$ the $\mathcal{O}_{\mathcal{L}}$-module of all $d$-dimensional derivations $D$ : $\mathcal{O}_{\mathcal{L}}^{d} \rightarrow W$ 
Consider the $d$ th exterior product $\bigwedge_{\mathcal{O}_{\mathcal{L}}}^{d} \Omega_{\mathcal{O}_{K}}\left(\mathcal{O}_{\mathcal{L}}\right)$ (cf. [18] Chapter $19 \S 1$ ). This is the $\mathcal{O}_{\mathcal{L}}$-module $\Omega_{\mathcal{O}_{K}}\left(\mathcal{O}_{\mathcal{L}}\right) \otimes \cdots \otimes \Omega_{\mathcal{O}_{K}}\left(\mathcal{O}_{\mathcal{L}}\right)$ divided out by the $\mathcal{O}_{\mathcal{L}}$-submodule generated by the elements

$$
x_{1} \otimes \cdots \otimes x_{d}
$$

where $x_{i}=x_{j}$ for some $i \neq j$. The symbols $x_{1} \otimes \cdots \otimes x_{d}$ will be denoted by

$$
x_{1} \wedge \cdots \wedge x_{d}
$$

instead. For $\bigwedge_{\mathcal{O}_{\mathcal{L}}}^{d} \Omega_{\mathcal{O}_{K}}\left(\mathcal{O}_{\mathcal{L}}\right)$ we consider the $d$-dimensional derivation $\mathrm{d}:\left(\mathcal{O}_{\mathcal{L}}^{d}\right) \rightarrow$ $\bigwedge_{\mathcal{O}_{\mathcal{L}}}^{d} \Omega_{\mathcal{O}_{K}}\left(\mathcal{O}_{\mathcal{L}}\right)$ that sends $\left(a_{1}, \ldots, a_{d}\right)$ to the wedge product $a_{1} \wedge \cdots \wedge a_{d}$. This $\mathcal{O}_{L^{-}}$ module is the universal object in the category of $d$-dimensional derivations of $\mathcal{O}_{L}$ over $\mathcal{O}_{K}$, i.e,

Proposition 6.2.1. If $D:\left(\mathcal{O}_{L}\right)^{d} \rightarrow W$ is a d-dimensonal derivation over $\mathcal{O}_{K}$ then there exist a homomorphism $\beta: \bigwedge_{\mathcal{O}_{\mathcal{L}}}^{d} \Omega_{\mathcal{O}_{K}}\left(\mathcal{O}_{\mathcal{L}}\right) \rightarrow W$ of $\mathcal{O}_{L}$-modules such that the diagram

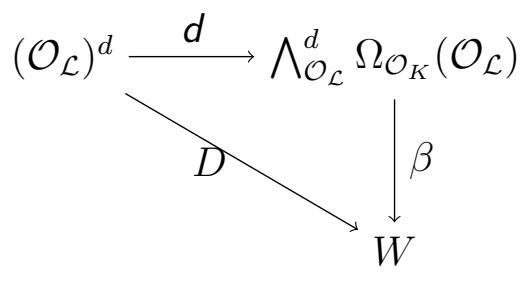

is commutative.

Proof. The homomorhpsim $\beta$ is clearly the homomorphism defined by $\beta\left(d a_{1} \wedge \cdots \wedge\right.$ 
$\left.d a_{d}\right)=D\left(a_{1}, \ldots, a_{d}\right)$.

Proposition 6.2.2. $\bigwedge_{\mathcal{O}_{\mathcal{L}}}^{d} \hat{\Omega}_{\mathcal{O}_{K}}\left(\mathcal{O}_{\mathcal{L}}\right) \cong\left(\mathcal{O}_{\mathcal{L}} / \mathcal{D}(L / K) \mathcal{O}_{\mathcal{L}}\right) d T_{1} \wedge \cdots \wedge d T_{d-1} \wedge d \pi_{L}$ as $\mathcal{O}_{\mathcal{L}}$-modules, where $\pi_{L}$ is a uniformizer for $L$ and $\mathcal{D}(L / K)$ is the different of the extension $L / K$. That is, $d T_{1} \wedge \cdots \wedge d T_{d-1} \wedge d \pi_{L}$ generates $\bigwedge_{\mathcal{O}_{\mathcal{L}}}^{d} \hat{\Omega}_{\mathcal{O}_{K}}\left(\mathcal{O}_{\mathcal{L}}\right)$ as an $\mathcal{O}_{\mathcal{L}}$-module

Proof. This follows immediately from Lemma 6.1.1.

Proposition 6.2.3. Let $D \in D_{K}^{d}\left(\mathcal{O}_{\mathcal{L}}^{d}, W\right)$, then $\mathcal{D}(L / K) D\left(T_{1}, \ldots, \pi_{L}\right)=0$. Let $\beta_{1}, \ldots, \beta_{d} \in \mathcal{O}_{\mathcal{L}}$, and $g_{i}\left(T_{1}, \ldots, T_{d-1}, T_{d}\right) \in \mathcal{O}_{\tilde{\mathcal{L}}}\left[T_{d}\right] i=1, \ldots, d$, such that $\beta_{i}=$ $g_{i}\left(T_{1}, \ldots, \pi_{L}\right)$. Then

$$
D\left(\beta_{1}, \ldots, \beta_{d}\right)=\operatorname{det}\left[\frac{\partial \beta_{i}}{\partial T_{j}}\right]_{i, j} D\left(T_{1}, \ldots, \pi_{L}\right),
$$

where $\frac{\partial}{\partial T_{k}}, k=1, \ldots, d$ are the derivations from Definition 6.1.2. Moreover, let $w \in W$ such that $\mathcal{D}(L / K) w=0$, then the map

$$
D\left(\beta_{1}, \ldots, \beta_{d}\right):=\operatorname{det}\left[\frac{\partial \beta_{i}}{\partial T_{j}}\right]_{i, j} w
$$

is well-defined and belongs to $D_{K}^{d}\left(\mathcal{O}_{\mathcal{L}}^{d}, W\right)$. In other words, the map

$$
D \mapsto D\left(T_{1}, \ldots, \pi_{L}\right),
$$

defines an isomorphism from $D_{K}^{d}\left(\mathcal{O}_{\mathcal{L}}^{d}, W\right)$ to the $\mathcal{D}(L / \tilde{L})$-torsion subgroup of $W$. 
Proof. This follows Proposition 6.2.2 and the fact that

$$
D_{\mathcal{O}_{K}}^{d}\left(\mathcal{O}_{\mathcal{L}}, W\right) \cong \operatorname{Hom}_{\mathcal{O}_{K}}\left(\bigwedge_{\mathcal{O}_{\mathcal{L}}}^{d} \hat{\Omega}_{\mathcal{O}_{K}}\left(\mathcal{O}_{\mathcal{L}}\right), W\right)
$$




\section{Chapter 7}

\section{Deduction of the formulas}

In this section $M$ will denote a local field containing $K_{t}=K\left(\kappa_{t}\right), \pi_{M}$ a unifromizer for $M$ and $\pi_{t}$ a uniformizer for $K_{t}$.

\subsection{Description of the map $\psi_{\mathcal{M}, m}^{i}$ in terms of deriva- tions}

Proposition 7.1.1. Let $\mathcal{M}=M\left\{\left\{T_{1}\right\}\right\} \cdots\left\{\left\{T_{d-1}\right\}\right\}, M \supset K_{t}$, with $(m, t)$ admissible. Suppose $\pi^{m}$ divides $D(M / K)$; Proposition 6.1.4 guarantees that this happens when $(t-m) / \alpha \geq c_{1}$. Then the reduction

$$
D_{\mathcal{M}, m}^{i}: \mathcal{O}_{\mathcal{M}}^{d} \rightarrow \frac{R_{\mathcal{M}, 1}}{\frac{\pi^{m}}{\pi_{M}} R_{\mathcal{M}, 1}}
$$

of $D_{\mathcal{M}, m}^{i}$ to $R_{\mathcal{M}, 1} /\left(\pi^{m} / \pi_{M}\right) R_{\mathcal{M}, 1}$ is a d-dimensional derivation over $\mathcal{O}_{K}$. 
Proof. Let us fix an $a_{1}, \ldots, a_{d-1} \in \mathcal{O}_{\mathcal{M}}^{*}$. From Proposition 6.1 .2 and the fact

$$
D(M / K) D_{M, m}^{i}\left(a_{1}, \ldots, a_{d-1}, \pi_{M}\right)=0 \quad\left(\bmod \pi^{m} R_{\mathcal{M}, 1}\right)
$$

we can to construct, by Proposition 6.1.2, a derivation

$$
D: \mathcal{O}_{M} \rightarrow R_{\mathcal{M}, 1} / \pi^{m} R_{\mathcal{M}, 1}
$$

such that $D\left(\pi_{M}\right)=D_{\mathcal{M}, m}^{i}\left(a_{1}, \ldots, a_{d-1}, \pi_{M}\right)$ and $D\left(T_{k}\right)=D_{\mathcal{M}, m}^{i}\left(a_{1}, \ldots, a_{d-1}, T_{k}\right)$, $k=1, \ldots, d-1$, in the following way

$$
D(\alpha)=\sum_{k=1}^{d-1} \frac{\partial \alpha}{\partial T_{k}} D\left(T_{k}\right)+\frac{\partial \alpha}{\partial T_{d}} D\left(\pi_{M}\right)
$$

where $\alpha \in \mathcal{O}_{\mathcal{M}}$.

According to Proposition 5.4 .5 both $D$ and $D_{\mathcal{M}, m}^{i}\left(a_{1}, \ldots, a_{d-1}, \cdot\right)$ coincide in $\mu_{\mathcal{M}}$.

But from the Leibniz rule it follows, by comparing $D\left(\pi_{M} x\right)$ and $D_{\mathcal{M}, m}^{i}\left(a_{1}, \ldots, a_{d-1}, \pi_{M} x\right)$ when $x \in \mathcal{O}_{\mathcal{M}}$, that they coincide $\left(\bmod \left(\pi^{m} / \pi_{M}\right) R_{\mathcal{M}, 1}\right)$ in all $\mathcal{O}_{\mathcal{M}}$.

It follows now that

$$
D_{\mathcal{M}, m}^{i}: \mathcal{O}_{\mathcal{M}}^{d} \rightarrow \frac{R_{\mathcal{M}, 1}}{\frac{\pi^{m}}{\pi_{M}} R_{\mathcal{M}, 1}}
$$

satisfies all conditions from definition 6.2.1 and so by Proposition 6.2.3 we have that it is a $d$-dimensional derivation such that

$$
D_{\mathcal{M}, m}^{i}\left(\beta_{1}, \ldots, \beta_{d}\right)=\operatorname{det}\left[\frac{\partial \beta_{i}}{\partial T_{j}}\right]_{i, j} D_{\mathcal{M}, m}\left(T_{1}, \ldots, T_{d-1}, \pi_{M}\right)
$$


where $\beta_{1}, \ldots, \beta_{d} \in \mathcal{O}_{\mathcal{M}}$.

We can express the map $\psi_{\mathcal{M}, m}^{i}$ out of $D_{\mathcal{M}, m}^{i}: \mathcal{O}_{\mathcal{M}}^{d} \rightarrow R_{\mathcal{M}, 1} /\left(\pi^{m} / \pi_{M}\right) R_{\mathcal{M}, 1}$ in the following way

$$
\left\{\begin{aligned}
\psi_{\mathcal{M}, m}^{i}\left(u_{1}, \ldots, u_{d-1}, \pi_{M}\right) & =\frac{D_{\mathcal{M}, m}^{i}\left(u_{1}, \ldots, u_{d-1}, \pi_{M}\right)}{u_{1} \cdots u_{d-1} \pi_{M}} \quad\left(\bmod \frac{\pi^{m}}{\pi_{M}^{2}} R_{\mathcal{M}, 1}\right) \\
\psi_{\mathcal{M}, m}^{i}\left(u_{1}, \ldots, u_{d}\right) & =\frac{D_{\mathcal{M}, m}^{i}\left(u_{1}, \ldots, u_{d}\right)}{u_{1} \cdots u_{d}}\left(\bmod \frac{\pi^{m}}{\pi_{M}} R_{\mathcal{M}, 1}\right) \\
\psi_{\mathcal{M}, m}^{i}\left(u_{1}, \ldots, \pi_{M}^{k} u_{d}\right) & =k \psi_{\mathcal{M}, m}^{i}\left(u_{1}, \ldots, \pi_{M}\right)+\psi_{\mathcal{M}, m}^{i}\left(u_{1}, \ldots, u_{d}\right), k \in \mathbb{Z} \\
\psi_{\mathcal{M}, m}^{i}\left(a_{1}, \ldots, a_{i}, \ldots, a_{j}, \ldots, a_{d}\right) & =0, \text { whenever } a_{i}=a_{j} \text { for } i \neq j, a_{1} \ldots, a_{d} \in \mathcal{M}^{*}
\end{aligned}\right.
$$

where $u_{1}, \ldots, u_{d}$ are in $\mathcal{O}_{\mathcal{M}}^{*}=\left\{x \in \mathcal{O}_{\mathcal{M}}: v_{\mathcal{M}}(x)=0\right\}$. It is clear form the definition that this is independent from the choice of a uniformizer $\pi_{M}$ of $M$. Notice that the fourth property says that $\psi_{\mathcal{M}, m}^{i}$ is alternate, in particular it is skew-symmetric, i.e.,

$$
\psi_{\mathcal{M}, m}^{i}\left(a_{1}, \ldots, a_{i}, \ldots, a_{j}, \ldots, a_{d}\right)=-\psi_{\mathcal{M}, m}^{i}\left(a_{1}, \ldots, a_{j}, \ldots, a_{i}, \ldots, a_{d}\right)
$$

whenever $i \neq j$.

Let $\mathcal{L}=L\left\{\left\{T_{1}\right\}\right\} \cdots\left\{\left\{T_{d-1}\right\}\right\}$ and $v$ denote the normalized valuation $v_{\mathcal{L}} / v_{\mathcal{L}}(p)$. Let $\kappa_{L}=\kappa \cap L$, i.e., the subgroup of torsion points contained in $\mathcal{L}$. We denoted by $T_{\mathcal{L}}$ be the image of $F\left(\mu_{\mathcal{L}}\right)$ under the logarithm of the formal group $F$. Then we have a continuous isomorphism $l_{F}: F\left(\mu_{\mathcal{L}}\right) / \kappa_{L} \rightarrow T_{\mathcal{L}}$ and by Lemma 5.1 .3 we have the 
embedding

$$
R_{\mathcal{L}} / \pi^{n} R_{\mathcal{L}} \rightarrow \operatorname{Hom}_{C}^{\text {seq }}\left(T_{\mathcal{L}}, C / \pi^{n} C\right) \stackrel{\sim}{\rightarrow} \operatorname{Hom}_{C}^{s e q}\left(F\left(\mu_{\mathcal{L}}\right) / \kappa_{L}, C / \pi^{n} C\right)
$$

Proposition 7.1.2. Let $m$ such that $v\left(f^{(m-n)}(x)\right)>1 /(p-1)$ for all $x \in F\left(\mu_{\mathcal{L}}\right)$. Let $(m, t)$ be admissible and put $\mathcal{M}=L_{t}\left\{\left\{T_{1}\right\}\right\} \cdots\left\{\left\{T_{d-1}\right\}\right\}$ and $\mathcal{L}=L\left\{\left\{T_{1}\right\}\right\} \cdots\left\{\left\{T_{d-1}\right\}\right\}$. Then $\operatorname{Tr}_{\mathcal{M} / \mathcal{L}}$ induces a homomorphism from $R_{\mathcal{M}, 1} / \pi^{m} R_{\mathcal{M}, 1}$ to $\mathcal{L} / \pi^{n} R_{\mathcal{L}}$ and we have the representation

$$
\left(N_{\mathcal{M} / \mathcal{L}}(\alpha), x\right)_{\mathcal{L}, n}^{i}=\mathbb{T}_{\mathcal{L} / S}\left(\operatorname{Tr}_{\mathcal{M} / \mathcal{L}}\left(\psi_{\mathcal{M}, m}^{i}(\alpha)\right) l_{F}(x)\right), \quad \forall \alpha \in K_{d}(\mathcal{M}), \forall x \in F\left(\mu_{\mathcal{L}}\right) .
$$

In particular, $\operatorname{Tr}_{\mathcal{M} / \mathcal{L}}\left(\psi_{\mathcal{M}, m}^{i}(\alpha)\right)$ belongs to $R_{\mathcal{L}} / \pi^{n} R_{\mathcal{L}}$ and it is the unique element satisfying (7.3).

Proof. Since $e_{n}^{i}=f^{(m-n)}\left(e_{m}^{i}\right)$ and $f^{(m-n)}(x) \in \mu_{\mathcal{L}, 1} \subset \mu_{\mathcal{M}, 1}$, then

$$
\begin{aligned}
\left(N_{\mathcal{M} / \mathcal{L}}(\alpha), x\right)_{\mathcal{L}, n}^{i} & =\frac{1}{\pi^{m-n}}\left(\alpha, f^{(m-n)}(x)\right)_{\mathcal{M}, m}^{i} \\
& =\frac{1}{\pi^{m-n}} \mathbb{T}_{\mathcal{M} / S}\left(\psi_{\mathcal{M}, m}^{i}(\alpha) l\left(f^{(m-n)}(x)\right)\right) \\
& =\mathbb{T}_{\mathcal{L} / S}\left(\left[\operatorname{Tr}_{\mathcal{M} / \mathcal{L}}\left(\psi_{\mathcal{M}, m}^{i}(\alpha)\right)\right] l(x)\right)
\end{aligned}
$$

From the condition on $m$ we have that $\pi^{m-n} T_{\mathcal{L}} \subset T_{\mathcal{L}, 1}$. Thus, after taking duals with respect to $\mathbb{T}_{\mathcal{L} / S}$ we obtain

$$
\frac{1}{\pi^{m-n}} R_{\mathcal{L}} \supset R_{\mathcal{L}, 1}
$$


or,

$$
\pi^{n} R_{\mathcal{L}} \supset \pi^{m} R_{\mathcal{L}, 1}
$$

Then from $\operatorname{Tr}_{\mathcal{M} / \mathcal{L}}\left(R_{\mathcal{M}, 1}\right) \subset R_{\mathcal{L}, 1}$, cf. Proposition 5.2 .2 (1), it follows that

$$
\operatorname{Tr}_{\mathcal{M} / \mathcal{L}}\left(\pi^{m} R_{\mathcal{M}, 1}\right) \subset \pi^{m} R_{\mathcal{L}, 1} \subset \pi^{n} R_{\mathcal{L}}
$$

It then follows that $\operatorname{Tr}_{\mathcal{M} / \mathcal{L}}\left(\psi_{\mathcal{M}, m}^{i}(\alpha)\right) \in L / \pi^{n} R_{\mathcal{L}}$. Moreover, since $\left(N_{\mathcal{M} / \mathcal{L}}(\alpha), x\right)_{\mathcal{L}, n}^{i}$ belongs to $C / \pi^{n} C$ then $\operatorname{Tr}_{\mathcal{M} / \mathcal{L}}\left(\psi_{\mathcal{M}, m}^{i}(\alpha)\right) \in R_{\mathcal{L}} / \pi^{n} R_{\mathcal{L}}$. The uniqueness follows from Lemma 5.1.3.

Remark 7.1.1. For $m$ in Proposition 7.1.2 we can take any

$$
m>n+\alpha \log _{p}\left(\frac{v_{L}(p)}{p-1}\right)+\alpha \frac{1}{p-1} .
$$

This follows the same proof of Proposition 6.2 in [14]. Indeed, let $k=m-n$. Then $f^{(k) \prime}$ is divisible by $\pi^{k}$, which implies that every term $a_{i} X^{i}$ of the series $f^{(k)}$ satisfies $v\left(a_{i}\right)+v(i) \geq k / \alpha$. If $v\left(a_{i}\right)>1 /(p-1)$ then there is nothing to prove. If on the other hand $v\left(a_{i}\right) \leq 1 /(p-1)$ then $v(i) \geq k / \alpha-1 /(p-1)$. In this case

$$
v\left(x^{i}\right) \geq \frac{i}{v_{L}(p)} \geq \frac{p^{\frac{k}{\alpha}-\frac{1}{p-1}}}{v_{L}(p)}>\frac{1}{p-1}
$$


for all $x \in \mu_{\mathcal{L}}$, since $k / \alpha-1 /(p-1)>\log _{p}\left(v_{L}(p) /(p-1)\right)$. Then

$$
v\left(f^{(k)}(x)\right)>\frac{1}{p-1}
$$

for all $x \in \mu_{\mathcal{L}}$.

\subsection{Invariants of the representation $\tau$}

Recall that we have fixed a basis $\left\{e^{i}\right\}_{i=1}^{h}$ for $\lim _{\longleftarrow} \kappa_{n}$. We also denoted by $e_{n}^{i}$ the reduction of $e^{i}$ to $\kappa_{n}$. Clearly $\left\{e_{n}^{i}\right\}$ is a basis for $\kappa_{n}$.

Let $M$ be a finite extension of $K$ and let $\mathcal{M}=M\left\{\left\{T_{1}\right\}\right\} \cdots\left\{\left\{T_{d-1}\right\}\right\}$. Denote by $T(\kappa)$ be the Tate-module $\lim _{\longleftarrow} \kappa_{n}$. The action of $G_{\mathcal{M}}=\operatorname{Gal}(\overline{\mathcal{M}} / \mathcal{M})$ on $T(\kappa)$ defines a continuous representation

$$
\tau: G_{\mathcal{M}} \rightarrow G L_{h}(C)
$$

Let $\mathcal{M}_{n}=M_{n}\left\{\left\{T_{1}\right\}\right\} \cdots\left\{\left\{T_{d-1}\right\}\right\}$. The reduction of $\tau$ to $G L_{h}\left(C / \pi^{n} C\right)$ is the analogous representation of $G_{\mathcal{M}}$ on $\kappa_{n}$ and will be denoted by $\tau_{n}$. This clearly induces an embedding $\tau_{n}: G\left(\mathcal{M}_{n} / \mathcal{M}\right) \rightarrow G L_{h}\left(C / \pi^{n} C\right)$.

Assume $M \supset K_{t}$ where $\beta=(m, t)$ is admissible. If $a \in \mathcal{M}^{*}$, then

$$
\tau_{m+t}\left(\Upsilon_{\mathcal{M}}\left(\left\{T_{1}, \ldots, T_{d-1}, a\right\}\right)\right)
$$

is congruent to the identity matrix $I\left(\bmod \pi^{t}\right)$ because the Galois group $G\left(\mathcal{M}_{m+t} / \mathcal{M}\right)$ 
fixes $\kappa_{t}$ and so correspond in $G L_{h}\left(C / \pi^{m+t} C\right)$ to matrices $\equiv I\left(\bmod \pi^{t}\right)$, i.e., there exist characters $\chi_{\mathcal{M}, \beta: i, j}: \mathcal{M}^{*} \rightarrow C / \pi^{m} C$ such that

$$
\tau_{m+t}\left(\Upsilon_{\mathcal{M}}\left(\left\{T_{1}, \ldots, T_{d-1}, a\right\}\right)\right)=I+\pi^{t}\left(\chi_{\mathcal{M}, \beta: i, j}(a)\right) \in G L_{h}\left(C / \pi^{m+t} C\right) .
$$

For $M=K_{t}$ we simply write $\chi_{\beta: i, j}$. By Proposition 4.1.2 (4) we have that

$$
N_{\mathcal{M} / \mathcal{K}_{t}}\left\{T_{1}, \ldots, T_{d-1}, a\right\}=\left\{T_{1}, \ldots, T_{d-1}, N_{\mathcal{M} / \mathcal{K}_{t}}(a)\right\}
$$

where $\mathcal{K}_{t}=K_{t}\left\{\left\{T_{1}\right\}\right\} \cdots\left\{\left\{T_{d-1}\right\}\right\}$, and Proposition 4.2.1 (4) implies

$$
\chi_{\mathcal{M}, \beta: i, j}(a)=\chi_{\beta: i, j}\left(N_{\mathcal{M} / \mathcal{K}_{t}}(a)\right)
$$

The definition of the pairing $(,)_{\mathcal{M}, m}$ implies, for $v \in \kappa_{t}$, that

$$
\left(\left(\left\{T_{1}, \ldots, T_{d-1}, a\right\}, v\right)_{\mathcal{M}, m}^{i}\right)=\left(\chi_{\mathcal{M}, \beta: i, j}(a)\right)\left(v^{j}\right)
$$

here $\left(v^{j}\right)$ are the coordinates of $v$. In particular, for $v=e_{t}^{j}$ we have

$$
\left(\left\{T_{1}, \ldots, T_{d-1}, a\right\}, e_{t}^{j}\right)_{\mathcal{M}, m}^{i}=\chi_{\mathcal{M}, \beta: i, j}(a)
$$

According to Proposition 5.3.1 we see that $\chi_{\mathcal{M}: i, j}$ uniquely determines on $V_{\mathcal{M}, 1}$ a constant $c_{\mathcal{M}, \beta: i, j} \in R_{\mathcal{M}, 1} / \pi^{m} R_{\mathcal{M}, 1}$ such that

$$
\chi_{\mathcal{M}, \beta: i, j}(u)=\mathbb{T}_{\mathcal{M} / S}\left(\log (u) c_{\mathcal{M}, \beta: i, j}\right) \quad \forall u \in V_{\mathcal{M}, 1}
$$

Namely, $\rho_{\mathcal{M}, m}^{i}\left(T_{1}, \ldots, T_{d-1}, e_{t}^{j}\right)=c_{\mathcal{M}, \beta: i, j}$. Observe that by equation $7.6 c_{\mathcal{M}, \beta: i, j}$ is 
the image of $c_{\beta, i, j}:=c_{\mathcal{K}_{t}, \beta: i, j}$, where $\mathcal{K}_{t}=K_{t}\left\{\left\{T_{1}\right\}\right\} \cdots\left\{\left\{T_{d-1}\right\}\right\}$, under the map

$$
R_{\mathcal{K}_{t}, 1} / \pi^{m} R_{\mathcal{K}_{t}, 1} \rightarrow R_{\mathcal{M}, 1} / \pi^{m} R_{\mathcal{M}, 1}
$$

$\left(R_{\mathcal{K}_{t}, 1} \subset R_{\mathcal{M}, 1}\right)$. So we wil denote $c_{\mathcal{M}, \beta: i, j}$ by $\bar{c}_{\beta: i, j}$.

Finally, observe that $c_{\beta: i, j}$ is an invariant of the isomorphism class of $\left(F, e_{j}\right)$ because if $g:\left(F, e_{j}\right) \rightarrow\left(\tilde{F}, \tilde{e}_{j}\right)$ is such isomorphism then $\tilde{\rho}_{\mathcal{M}, m}^{i}\left(T_{1}, \ldots, T_{d-1}, g(x)\right)=$ $\rho_{\mathcal{M}, m}^{i}\left(T_{1}, \ldots, T_{d-1}, x\right)$

From Proposition 5.3 .2 we conclude that

Proposition 7.2.1. Let $M \supset K_{t},(m, t)$ admissible and $\mathcal{M}=M\left\{\left\{T_{1}\right\}\right\} \cdots\left\{\left\{T_{d-1}\right\}\right\}$. If $r(X)$ is a t-normalized series for $F$, then

$$
\begin{aligned}
D_{\mathcal{M}, m}^{i}\left(T_{1}, \ldots, T_{d-1}, r\left(e_{t}^{j}\right)\right) & =\psi_{\mathcal{M}, m}^{i}\left(T_{1}, \ldots, T_{d-1}, r\left(e_{t}^{j}\right)\right) r\left(e_{t}^{j}\right) T_{1} \cdots T_{d-1} \\
& =-r^{\prime}\left(e_{t}^{j}\right) T_{1} \cdots T_{d-1} \frac{\bar{c}_{\beta: i, j}}{l^{\prime}\left(e_{t}^{j}\right)}
\end{aligned}
$$

Let $L$ be a local field and let $\mathcal{L}=L\left\{\left\{T_{1}\right\}\right\} \cdots\left\{\left\{T_{d-1}\right\}\right\}$. Define

$$
R_{\mathcal{L}, 1}^{\prime}:=\left\{x \in \mathcal{L}: v_{\mathcal{L}}(x) \geq-v_{L}(D(L / S))-\left[\frac{v_{L}(p)}{p-1}\right]\right\}
$$

Note that

$$
v_{\mathcal{L}}(x) \geq-v_{L}(D(L / S))-\left[\frac{v_{L}(p)}{p-1}\right]
$$

if and only if

$$
v_{\mathcal{L}}(x) \geq-v_{L}(D(L / S))-\left(\frac{v_{L}(p)}{p-1}\right)
$$


This holds since $v_{\mathcal{L}}(x)$ and $v_{L}(D(L / S))$ are integers, therefore the conditions

$$
\left[\frac{v_{L}(p)}{p-1}\right] \geq-v_{L}(D(L / S))-v_{\mathcal{L}}(x)
$$

and

$$
\left(\frac{v_{L}(p)}{p-1}\right) \geq-v_{L}(D(L / S))-v_{\mathcal{L}}(x)
$$

are equivalent by the very definition of the integral part of a real number.

Comparing with equations $(7.9)$ and $(5.4)$ we see that $R_{\mathcal{L}, 1}^{\prime}=\pi_{L} R_{\mathcal{L}, 1}$.

If $M / L$ is a finite extension of local fields and we put $\mathcal{M}=M\left\{\left\{T_{1}\right\}\right\} \cdots\left\{\left\{T_{d-1}\right\}\right\}$ and $\mathcal{L}=L\left\{\left\{T_{1}\right\}\right\} \cdots\left\{\left\{T_{d-1}\right\}\right\}$, then clearly

$$
R_{\mathcal{M}, 1}^{\prime}=(1 / D(M / L)) R_{\mathcal{L}, 1}^{\prime}
$$

Proposition 7.2.2. Let $\beta=(k, t)$ be an admissible pair such that $\pi^{k} \mid D\left(K_{t} / K\right)$; this happens for example when $(t-k) / \alpha \geq c_{1}$; this $c_{1}$ is the constant from Proposition 6.1.4. Let $a_{j} \in \mathcal{O}_{K_{t}}$ such that $d e_{t}^{j}=a_{j} d \pi_{t}$ in $\Omega_{\mathcal{O}_{K}}\left(\mathcal{O}_{K_{t}}\right) ; \pi_{t}$ is a uniformizer for $K_{t}$.

Then

$$
c_{\beta: i, j} \in \frac{a_{j} R_{\mathcal{K}_{t}, 1}^{\prime}+\frac{\pi^{k}}{\pi_{t}} R_{\mathcal{K}_{t}, 1}^{\prime}+\pi^{k} R_{\mathcal{K}_{t}, 1}}{\pi^{k} R_{\mathcal{K}_{t}, 1}},
$$

where $\mathcal{K}_{t}=K_{t}\left\{\left\{T_{1}\right\}\right\} \cdots\left\{\left\{T_{d-1}\right\}\right\}$.

Proof. Following the proof of Proposition 6.5 of [14]. We begin by taking a repre- 
sentative $\lambda_{i, j}$ of $c_{\beta: i, j}$ in $R_{\mathcal{K}_{t}, 1}$. We have to show that

$$
\lambda_{i, j} \in a_{j} R_{\mathcal{K}_{t}, 1}^{\prime}+\left(\frac{\pi^{k}}{\pi_{t}}\right) R_{\mathcal{K}_{t}, 1}^{\prime}
$$

Let $M \supset K_{t}, \pi_{M}$ and $\pi_{t}$ uniformizers for $M$ and $K_{t}$, respectively, and $\mathcal{M}=$ $M\left\{\left\{T_{1}\right\}\right\} \cdots\left\{\left\{T_{d-1}\right\}\right\}, \mathcal{K}_{t}=\mathcal{K}_{t}\left\{\left\{T_{1}\right\}\right\} \cdots\left\{\left\{T_{d-1}\right\}\right\}$. Let $b \in \mathcal{O}_{M}$ such that $d \pi_{t}=$ $b d \pi_{M}$; this exist by Proposition 6.1.3. Then $D\left(M / K_{t}\right)=b \mathcal{O}_{M}$. Set $\beta_{j}=b a_{j} \in \mathcal{O}_{M}$. Clearly, $d e_{t}^{j}=\beta_{j} d \pi_{M}$. By Proposition 7.1.1.

$$
D_{\mathcal{M}, k}^{i}: \mathcal{O}_{\mathcal{M}}^{d} \rightarrow R_{\mathcal{M}, 1} /\left(\pi^{k} / \pi_{M}\right) R_{\mathcal{M}, 1}
$$

is a $d$-dimensional derivation over $\mathcal{O}_{K}$, which together with Proposition 7.2.1 implies

$$
\begin{aligned}
r^{\prime}\left(e_{t}^{j}\right) \beta_{j} D_{\mathcal{M}, k}^{i}\left(T_{1}, \ldots, T_{d-1}, \pi_{M}\right) & =D_{\mathcal{M}, k}^{i}\left(T_{1}, \ldots, T_{d-1}, r\left(e_{t}^{j}\right)\right) \\
& =-r^{\prime}\left(e_{t}^{j}\right) T_{1} \cdots T_{d-1} \frac{\bar{c}_{\beta: i, j}}{l^{\prime}\left(e_{t}^{j}\right)}\left(\bmod \left(\pi^{k} / \pi_{M}\right) R_{\mathcal{M}, 1}\right) .
\end{aligned}
$$

Recall that $\bar{c}_{\beta: i, j}$ is the image of $c_{\beta: i, j}$ under the map $R_{\mathcal{K}_{t}, 1} / \pi^{k} R_{\mathcal{K}_{t}, 1} \rightarrow R_{\mathcal{M}, 1} / \pi^{k} R_{\mathcal{M}, 1}$; $R_{\mathcal{K}_{t}, 1} \subset R_{\mathcal{M}, 1}$. This identity implies

$$
\lambda_{i, j} \in \beta_{j} R_{\mathcal{M}, 1}+\left(\frac{\pi^{k}}{\pi_{M}}\right) R_{\mathcal{M}, 1} .
$$


Then

$$
\begin{aligned}
v_{\mathcal{M}}\left(\lambda_{i, j}\right) & \geq \min \left\{v_{\mathcal{M}}\left(\beta_{j} R_{\mathcal{M}, 1}\right), v_{\mathcal{M}}\left(\left(\pi^{k} / \pi_{M}\right) R_{\mathcal{M}, 1}\right)\right\} \\
& \geq \min \left\{v_{\mathcal{M}}\left(\beta_{j}\right)-v_{\mathcal{M}}(D(M / S))-\frac{e(M)}{p-1}-1,\right. \\
& \left.v_{\mathcal{M}}\left(\frac{\pi^{k}}{\pi_{M}}\right)-v_{\mathcal{M}}(D(M / S))-\frac{e(M)}{p-1}-1\right\}
\end{aligned}
$$

We will further assume that $M$ is the local field obtained by adjoining to $K_{t}$ the roots of the Eisenstein polynomial $X^{n}-\pi_{t},(n, p)=1$. Then $e(M)=n e\left(K_{t}\right)$ and $D\left(M / K_{t}\right)=n \pi_{M}^{n-1}=\pi_{t} / \pi_{M}$

$$
\begin{aligned}
v_{\mathcal{M}}\left(\lambda_{i, j}\right) \geq \min \left\{v_{\mathcal{M}}\left(a_{j}\right)-v_{\mathcal{M}}\left(D\left(K_{t} / S\right)\right)-\frac{e(M)}{p-1}-1,\right. \\
\left.v_{\mathcal{M}}\left(\frac{\pi^{k}}{\pi_{t}}\right)-v_{\mathcal{M}}\left(D\left(K_{t} / S\right)\right)-\frac{e(M)}{p-1}-1\right\}
\end{aligned}
$$

Dividing everything by $e\left(M / K_{t}\right)=n$ and noticing that $v_{\mathcal{M}}(x)=e\left(M / K_{t}\right) v_{\mathcal{K}_{t}}(x)$ for $x \in \mathcal{K}_{t}$ we obtain

$$
\begin{array}{r}
v_{\mathcal{K}_{t}}\left(\lambda_{i, j}\right) \geq \min \left\{v_{\mathcal{K}_{t}}\left(a_{j}\right)-v_{\mathcal{K}_{t}}\left(D\left(K_{t} / S\right)\right)-\frac{e\left(K_{t}\right)}{p-1}-\frac{1}{n},\right. \\
\left.v_{\mathcal{K}_{t}}\left(\frac{\pi^{k}}{\pi_{K_{t}}}\right)-v_{\mathcal{K}_{t}}\left(D\left(K_{t} / S\right)\right)-\frac{e\left(K_{t}\right)}{p-1}-\frac{1}{n}\right\}
\end{array}
$$

Letting $n \rightarrow \infty$ we obtain

$$
\begin{array}{r}
v_{\mathcal{K}_{t}}\left(\lambda_{i, j}\right) \geq \min \left\{v_{\mathcal{K}_{t}}\left(a_{j}\right)-v_{\mathcal{K}_{t}}\left(D\left(K_{t} / S\right)\right)-\frac{e\left(K_{t}\right)}{p-1},\right. \\
\left.v_{\mathcal{K}_{t}}\left(\frac{\pi^{k}}{\pi_{t}}\right)-v_{\mathcal{K}_{t}}\left(D\left(K_{t} / S\right)\right)-\frac{e\left(K_{t}\right)}{p-1}\right\}
\end{array}
$$


which implies (7.11).

Lemma 7.2.1. Assume $\pi^{k} \mid D\left(K_{t} / K\right), \beta=(k, t)$ admissible, and $\mathcal{M}=M\left\{\left\{T_{1}\right\}\right\} \cdots\left\{\left\{T_{d-1}\right\}\right\}$ with $M \supset K_{t}$. Moreover, assume that $j$ is such that the inequality in Proposition 6.1 .5 (2) holds. Then there exist a d-dimensional derivation over $\mathcal{O}_{K}$

$$
D: \mathcal{O}_{\mathcal{M}}^{d} \rightarrow \frac{R_{\mathcal{M}, 1}}{\frac{\pi^{k}}{\pi_{M}} R_{\mathcal{M}, 1}}
$$

such that $D\left(T_{1}, \ldots, T_{d-1}, e_{t}^{j}\right)=-T_{1} \cdots T_{d-1} \bar{c}_{\beta: i, j} / l^{\prime}\left(e_{t}^{j}\right)$. Moreover, all such derivations coincide when reduced to

$$
R_{\mathcal{M}, 1} /\left(\pi^{m} / \pi_{M}\right) R_{\mathcal{M}, 1}
$$

for $m \leq k$, if $m / \alpha \leq k / \alpha-v(D(M / K))+t / \alpha-c_{1}$ ( $c_{1}$ is the constant from Proposition 6.1.4). Here $v$ denotes normalized valuation $v_{\mathcal{M}} / v_{\mathcal{M}}(p)$. In particular, they coincide with the reduction of $D_{\mathcal{M}, k}^{i}: \mathcal{O}_{\mathcal{M}}^{d} \rightarrow R_{\mathcal{M}, 1} /\left(\pi^{k} / \pi_{M}\right) R_{\mathcal{M}, 1}$ to $R_{\mathcal{M}, 1} /\left(\pi^{m} / \pi_{M}\right) R_{\mathcal{M}, 1}$.

Proof. Let $\beta_{j} \in \mathcal{O}_{M}$ such that $d e_{t}^{j}=\beta_{j} d \pi_{M}$ in $\Omega_{\mathcal{O}_{K}}\left(\mathcal{O}_{M}\right)$; this $\beta_{i}$ exists by Proposition 6.1.3. We shall prove that there exist a $\gamma_{j} \in R_{\mathcal{M}, 1} /\left(\pi^{k} / \pi_{M}\right) R_{\mathcal{M}, 1}$ such that $-\bar{c}_{\beta: i, j} / l^{\prime}\left(e_{t}^{j}\right)=\beta_{j} \gamma_{j}$, in particular $-T_{1} \cdots T_{d-1} \bar{c}_{i, j} / l^{\prime}\left(e_{j}^{t}\right)=\beta_{j} T_{1} \cdots T_{d-1} \gamma_{j}$. Then using Proposition 6.2 .3 we define

$$
D\left(\alpha_{1}, \ldots, \alpha_{d}\right):=\operatorname{det}\left[\frac{\partial \alpha_{i}}{\partial T_{j}}\right]_{1 \leq i, j \leq d} T_{1} \cdots T_{d-1} \gamma_{j},
$$

where $\alpha_{1}, \ldots, \alpha_{d} \in \mathcal{O}_{\mathcal{M}}$, which is the required multidimensional derivation since 
$D\left(T_{1}, \ldots, T_{d-1}, e_{t}^{j}\right)=\beta_{j} T_{1} \cdots T_{d-1} \gamma_{j}=-T_{1} \cdots T_{d-1} \bar{c}_{\beta: i, j} / l^{\prime}\left(e_{t}^{j}\right)$.

Let $\lambda_{j}$ be a representative for $c_{\beta: i, j}$ in $R_{\mathcal{K}_{t}, 1}$. By Proposition 7.2.2, $\lambda_{j} \in a_{j} R_{\mathcal{K}_{t}, 1}^{\prime}+$ $\left(\pi^{k} / \pi_{t}\right) R_{\mathcal{K}_{t}, 1}^{\prime}$ where $d e_{j}^{t}=a_{j} d \pi_{t}$. Let $d \pi_{t}=b d \pi_{M}$, in particular $D\left(M / K_{t}\right)=b \mathcal{O}_{M}$, and set $\beta_{j}=a_{j} b$. We have by 7.10 that

$$
\begin{gathered}
\frac{1}{b} R_{\mathcal{K}_{t}, 1}^{\prime}=\left(1 / D\left(M / K_{t}\right)\right) R_{\mathcal{K}_{t}, 1}^{\prime}=R_{\mathcal{M}, 1}^{\prime} \subset R_{\mathcal{M}, 1}, \\
\frac{\pi^{k}}{\pi_{t}} R_{\mathcal{K}_{t}, 1}^{\prime} \subset \frac{\pi^{k}}{\pi_{M}} \frac{D\left(M / K_{t}\right)}{\pi_{t} / \pi_{M}} R_{\mathcal{M}, 1}^{\prime} \subset \frac{\pi^{k}}{\pi_{M}} R_{\mathcal{M}, 1},
\end{gathered}
$$

where the last inclusion follows since $D\left(M / K_{t}\right)$ is divisible by $\pi_{t} / \pi_{M}$; this follows from the general inequality $v_{M}\left(D\left(M / K_{t}\right)\right) \geq e\left(M / K_{t}\right)-1$ (cf. [2] chap 1 prop 5.4). It thus follows

$$
-\frac{c_{\beta: i, j}}{l^{\prime}\left(e_{t}^{j}\right)}=-\frac{\lambda_{j}}{l^{\prime}\left(e_{t}^{j}\right)} \in \beta_{j} \frac{R_{\mathcal{M}, 1}}{\left(\pi^{k} / \pi_{M}\right) R_{\mathcal{M}, 1}} .
$$

Now let us prove the second assertion. Since $-c_{\beta: i, j} / l^{\prime}\left(e_{t}^{j}\right)=b_{j} \gamma_{j}$, then $\gamma_{j}$ is uniquely defined $\left(\bmod \left(\pi^{k} / \pi_{M} \beta_{j}\right) R_{\mathcal{M}, 1}\right)$. Let $m \leq k$, then the d-dimensional derivation over $\mathcal{O}_{K}$

$$
D: \mathcal{O}_{\mathcal{M}}^{d} \rightarrow \frac{R_{\mathcal{M}, 1}}{\frac{\pi^{m}}{\pi_{M}} R_{\mathcal{M}, 1}}
$$

such that $D\left(T_{1}, \ldots, T_{d-1}, \pi_{M}\right)=T_{1} \cdots T_{d-1} \overline{\gamma_{j}}$ is uniquely determined if $\pi^{m} \mid\left(\pi^{k} / \beta_{j}\right)$. Under this condition it follows now that the reduction of all the derivations (7.13) to $R_{\mathcal{M}, 1} /\left(\pi^{m} / \pi_{M}\right) R_{\mathcal{M}, 1}$ coincide. 
Finally, the condition $\pi^{m} \mid\left(\pi^{k} / \beta_{j}\right)$ is fulfill when

$$
m / \alpha \leq k / \alpha-v(D(M / K))+t / \alpha-c_{1}
$$

Indeed, $d e_{t}^{j}=\beta_{j} d \pi_{M}$ implies that $v\left(p_{t}\right)+v\left(\beta_{j}\right)=v(D(M / K))$, with $p_{t}$ as in Proposition $6.1 .4(2)$, thus by this same proposition

$$
\begin{aligned}
m / \alpha & \leq k / \alpha-v(D(M / K))+t / \alpha-c_{1} \\
& \leq k / \alpha-v(D(M / K))+v\left(p_{t}\right) \\
& =k / \alpha-v\left(\beta_{j}\right)
\end{aligned}
$$

which is precisely the condition $\pi^{m} \mid\left(\pi^{k} / \beta_{j}\right)$.

Proposition 7.2.3. Let $M \supset K_{t}$ and $\mathcal{M}=M\left\{\left\{T_{1}\right\}\right\} \cdots\left\{\left\{T_{d-1}\right\}\right\}$, with $\beta=(k, t)$ admissible and $(t-k) / \alpha \geq c_{1} ; c_{1}$ the constant from Proposition 6.1.4. Let $1 \leq j \leq h$ be as in Lemma 7.2.1. Let $m \leq k$ such that

$$
m / \alpha \leq k / \alpha+t / \alpha-v(D(M / K))-c_{1}
$$

Then the reduction of

$$
D_{\mathcal{M}, m}^{i}: \mathcal{O}_{\mathcal{M}}^{d} \rightarrow R_{\mathcal{M}, 1} / \pi^{m} R_{\mathcal{M}, 1}
$$

to $R_{\mathcal{M}, 1} /\left(\pi^{m} / \pi_{M}\right) R_{\mathcal{M}, 1}$ is a d-dimensional derivation over $\mathcal{O}_{K}$. Moreover, this $d$ dimensional derivation can be explicitly constructed as follows. Let de $e_{t}^{j}=\beta_{1} d \pi_{M}$ 
in $\Omega_{\mathcal{O}_{K}}\left(\mathcal{O}_{M}\right)$, so that there exists a uniquely determined $\bar{\gamma}_{i} \in R_{\mathcal{M}, 1} /\left(\pi^{m} / \pi_{M}\right) R_{\mathcal{M}, 1}$ such that that there is a lifting $\gamma_{i}$ of $\bar{\gamma}_{i}$ in $R_{\mathcal{M}, 1} /\left(\pi^{k} / \pi_{M}\right) R_{\mathcal{M}, 1}$ for which $\beta_{1} \gamma_{i}=$ $-\bar{c}_{\beta: i, j} / l^{\prime}\left(e_{t}^{j}\right)$. Then

$$
D_{\mathcal{M}, m}^{i}\left(T_{1}, \ldots, T_{d-1}, \pi_{M}\right)=T_{1} \cdots T_{d-1} \bar{\gamma}_{i}
$$

and

$$
D_{\mathcal{M}, m}^{i}\left(\alpha_{1}, \ldots, \alpha_{d}\right):=\operatorname{det}\left[\frac{\partial \alpha_{i}}{\partial T_{j}}\right]_{1 \leq i, j \leq d} T_{1} \cdots T_{d-1} \bar{\gamma}_{i},
$$

where $\alpha_{1}, \ldots, \alpha_{d} \in \mathcal{O}_{\mathcal{M}}$ and $\frac{\partial}{\partial T_{k}}, 1 \leq k \leq d$, are the derivations from Definition 6.1.2. In particular,

$$
D_{\mathcal{M}, m}^{i}\left(T_{1}, \ldots, T_{d-1}, e_{t}^{a}\right)=-T_{1} \cdots T_{d-1} \bar{c}_{\beta: i, j} / l^{\prime}\left(e_{t}^{a}\right), \quad \text { for all } 1 \leq a \leq h
$$

Proof. By Proposition $5.2 .2(3)$,

$$
D_{\mathcal{M}, m}^{i}: \mathcal{O}_{\mathcal{M}}^{d} \rightarrow R_{\mathcal{M}, 1} /\left(\pi^{m} / \pi_{M}\right) R_{\mathcal{M}, 1}
$$

is the reduction of

$$
D_{\mathcal{M}, k}^{i}: \mathcal{O}_{\mathcal{M}}^{d} \rightarrow R_{\mathcal{M}, 1} /\left(\pi^{k} / \pi_{M}\right) R_{\mathcal{M}, 1}
$$

and the latter is a $d$-dimensional derivation over $\mathcal{O}_{K}$ according to Proposition 7.1.1 and

$$
D_{\mathcal{M}, k}^{i}\left(T_{1}, \ldots, T_{d-1}, e_{t}^{j}\right)=-T_{1} \cdots T_{d-1} \bar{c}_{\beta: i, j} / l^{\prime}\left(e_{t}^{j}\right)
$$

according Proposition 7.2.1. Finally, by Proposition 6.1.4 the condition $(t-k) / \alpha \geq c_{1}$ 
implies that $\pi^{k} \mid D\left(K_{t} / K\right)$, and Lemma 7.2.1 provides a way of constructing explicitly a $d$-dimensional derivation

$$
D: \mathcal{O}_{\mathcal{M}}^{d} \rightarrow R_{\mathcal{M}, 1} /\left(\pi^{k} / \pi_{M}\right) R_{\mathcal{M}, 1}
$$

with $D\left(T_{1}, \ldots, T_{d-1}, e_{t}^{j}\right)=-T_{1} \cdots T_{d-1} \bar{c}_{\beta: i, j} / l^{\prime}\left(e_{t}^{j}\right)$; there exist a $\gamma_{j} \in R_{\mathcal{M}, 1} /\left(\pi^{k} / \pi_{M}\right) R_{\mathcal{M}, 1}$, by the proof of Lemma 7.2.1, such that $-\bar{c}_{\beta: i, j} / l^{\prime}\left(e_{t}^{j}\right)=\beta_{j} \gamma_{j}$ and we define

$$
D\left(\alpha_{1} \ldots, \alpha_{d}\right):=\operatorname{det}\left[\frac{\partial \alpha_{i}}{\partial T_{j}}\right]_{1 \leq i, j \leq d} T_{1} \cdots T_{d-1} \gamma_{j}
$$

where $\alpha_{1}, \ldots, \alpha_{d} \in \mathcal{O}_{\mathcal{M}}$. By this same lemma both $D$ and $D_{\mathcal{M}, k}^{i}$ coincide with $D_{\mathcal{M}, m}^{i}$ when reduced to

$$
R_{\mathcal{M}, 1} /\left(\pi^{m} / \pi_{M}\right) R_{\mathcal{M}, 1}
$$

The last statement holds because $D_{\mathcal{M}, k}^{i}$ is a multidimensional derivation and the formula for $D_{\mathcal{M}, k}^{i}\left(T_{1}, \ldots, T_{d-1}, r\left(e_{t}^{a}\right)\right)$ in equation 7.8 .

\subsection{Main formulas}

Theorem 7.3.1. Let $L \supset K_{n}$ and $\mathcal{L}=L\left\{\left\{T_{1}\right\}\right\} \cdots\left\{\left\{T_{d-1}\right\}\right\}$. Let

$$
m=n+2+\left[\alpha \log _{p}\left(\frac{v_{L}(p)}{p-1}\right)+\frac{\alpha}{p-1}\right]
$$

Take $k$ large enough such that 


$$
\begin{gathered}
t / \alpha+k / \alpha \geq m / \alpha+c_{1}+v(D(M / K)) \\
k+\alpha+1 \geq c_{1} \alpha
\end{gathered}
$$

where $t=2 k+\alpha+1, M=L_{t}$ and $\mathcal{M}=M\left\{\left\{T_{1}\right\}\right\} \cdots\left\{\left\{T_{d-1}\right\}\right\}$. This happens for example when

$$
k / \alpha \geq m / \alpha+\log _{2}(2 k+\alpha+1) /(p-1)+c_{1}+c_{2}+v(D(L / K)),
$$

where $c_{1}$ and $c_{2}$ are the constants from Proposition 6.1.4. Then $\mathcal{M}, t, k$, and $m$ satisfy Proposition 7.2.3. Let

$$
D_{\mathcal{M}, m}^{i}: \mathcal{O}_{\mathcal{M}}^{d} \rightarrow \frac{R_{\mathcal{M}, 1}}{\frac{\pi^{m}}{\pi_{M}} R_{\mathcal{M}, 1}}
$$

be the d-dimensional derivation constructed as in Proposition 7.2.3. and let

$$
\psi_{\mathcal{M}, m}^{i}: K_{d}(\mathcal{M}) \rightarrow \frac{R_{\mathcal{M}, 1}}{\frac{\pi^{m}}{\pi_{M}^{2}} R_{\mathcal{M}, 1}}
$$

be the multidimensional logarithmic derivative built out of $D_{\mathcal{M}, m}^{i}$ by equation (7.1). Then

$$
\left(N_{\mathcal{M} / \mathcal{L}}(\alpha), x\right)_{\mathcal{L}, n}^{i}=\mathbb{T}_{\mathcal{L} / S}\left(\operatorname{Tr}_{\mathcal{M} / \mathcal{L}}\left(\psi_{\mathcal{M}, m}^{i}(\alpha)\right) l(x)\right)=\mathbb{T}_{\mathcal{M} / S}\left(\psi_{\mathcal{M}, m}^{i}(\alpha) l(x)\right),
$$

for all $\alpha \in K_{d}(\mathcal{M})$ and all $x \in F\left(\mu_{\mathcal{L}}\right)$. 
Proof. By considering the tower $L_{t} \subset L \subset K$ and the upperbound in Proposition 6.1 .4 (1), we get

$$
v(D(M / K)) \leq v\left(L_{t} / L\right)+v(D(L / K)) \leq t / \alpha+\frac{\log _{2}(t)}{p-1}+c_{2}+v(D(L / K)) .
$$

Adding $m / \alpha+c_{1}$ we obtain, by (7.17), the inequality (7.15). The definition of $t$ clearly implies that $(k, t)$ is admissible and condition $(7.16)$ implies $(t-k) / \alpha \geq c_{1}$, thus $M, \mathcal{M}, t, k$ and $m$ satisfy the hypothesis of Proposition 7.2.3. The result now follows from Proposition 7.1.2, the Remark 7.1.1 and equation 7.1. It remains only to check that $\operatorname{Tr}_{\mathcal{M} / \mathcal{L}}\left(\left(\pi^{m} / \pi_{M}^{2}\right) R_{\mathcal{M}, 1}\right) \subset \pi^{n} R_{\mathcal{L}}$, so that $\operatorname{Tr}_{\mathcal{M} / \mathcal{L}}\left(\psi_{\mathcal{M}, m}^{i}(\alpha)\right)$ is well defined in $R_{\mathcal{L}} / \pi^{n} R_{\mathcal{L}}$. To do this notice that condition 7.14 implies

$$
m-1>n+\alpha \log _{p}\left(\frac{v_{L}(p)}{p-1}\right)+\frac{\alpha}{p-1}
$$

and we can apply Remark 7.1.1 to $m-1$ and get, by equation (7.4), that

$$
\operatorname{Tr}_{\mathcal{M} / \mathcal{L}}\left(\pi^{m-1} \frac{\pi}{\pi_{M}^{2}} R_{\mathcal{M}, 1}\right) \subset \operatorname{Tr}_{\mathcal{M L}}\left(\pi^{m-1} R_{\mathcal{M}, 1}\right) \subset \pi^{n} R_{\mathcal{L}}
$$

bearing in mind that $\pi_{M}^{2} \mid \pi$, since $\pi^{k} \mid D(M / K)$ implies $e(M / K)>1$ ( i.e., $M / K$ is not unramified).

We state a simplified version of the theorem above

Theorem 7.3.2. Let $L \supset K_{n}$ and $\mathcal{L}=L\left\{\left\{T_{1}\right\}\right\} \cdots\left\{\left\{T_{d-1}\right\}\right\}$. Let $\mathcal{M}$ and $m$ be 
defined as in Theorem 7.3.1. Then

$$
\left(N_{\mathcal{M} / \mathcal{L}}(\alpha), x\right)_{\mathcal{L}, n}^{i}=\mathbb{T}_{\mathcal{L} / S}\left(\operatorname{Tr}_{\mathcal{M} / \mathcal{L}}\left(\psi_{\mathcal{M}, m}^{i}(\alpha)\right) l(x)\right)=\mathbb{T}_{\mathcal{M} / S}\left(\psi_{\mathcal{M}, m}^{i}(\alpha) l(x)\right)
$$

for all $\alpha \in K_{d}(\mathcal{M})$ and all $x \in F\left(\mu_{\mathcal{L}}\right)$. Here $\psi_{\mathcal{M}, m}^{i}$ is the explicit multidimensional logarithmic derivative constructed in Theorem 7.3.1. 


\section{Chapter 8}

\section{Formulas for the Lubin-Tate formal groups}

In this section, guided in much by Section 7 in [14], we will give an explicit description of the corresponding formulas for the case where the formal group is Lubin-Tate. This will include an explicit computation of the invariants defined in equation (7.7) and of the lower bounds for $t, m$ and $k$ in Theorem 7.3.1.

Let $K / \mathbb{Q}_{p}$ be a local field with ring of integers $\mathcal{O}_{K}, \pi$ a uniformizer for $K, k_{K}$ its residue field and $q=\left|k_{K}\right|$. Let $\Lambda_{\pi}$ be the subset of $\mathcal{O}_{K}[[X]]$ consiting of the series $f$ such that

1. $f(X) \equiv \pi X(\bmod \operatorname{deg} 2)$.

2. $f(X) \equiv X^{q}(\bmod \pi)$.

Let $F_{f}$ be the Lubin-Tate formal group such that $[\pi]_{F_{f}}=f$. In this case we will take $S=K$ and $C=\mathcal{O}_{K}$. Evidently $f$ has height equal to 1 with respect to to 
$C=\mathcal{O}_{K}$. Thus $\kappa_{n} \simeq \mathcal{O}_{K} / \pi^{n} \mathcal{O}_{K}$ and $\lim _{\longleftarrow} \kappa_{n} \simeq \mathcal{O}_{K}$. Let $e$ be a generator for $\lim _{\longleftarrow} \kappa_{n}$ and $e_{n}$ its reduction in $\kappa_{n}$.

Let $K_{n}=K\left(\kappa_{n}\right)$ and $K_{\infty}=K(\kappa)$, where $\kappa=\cup \kappa_{n}$. Let $\mathcal{K}_{n}=K_{n}\left\{\left\{T_{1}\right\}\right\} \cdots\left\{\left\{T_{d-1}\right\}\right\}$ and $\mathcal{K}=K\left\{\left\{T_{1}\right\}\right\} \cdots\left\{\left\{T_{d-1}\right\}\right\}$. The extension $K_{n} / K$ is totally ramified and $e_{n}$ is a uniformizer for $K_{n}$. Moreover, $\left[K_{n} / K\right]=q^{n}-q^{n-1}$, then the imbedding $\tau_{n}$

$$
\tau_{n}: \operatorname{Gal}\left(K_{n}\left\{\left\{T_{1}\right\}\right\} \cdots\left\{\left\{T_{d-1}\right\}\right\} / K\left\{\left\{T_{1}\right\}\right\} \cdots\left\{\left\{T_{d-1}\right\}\right\}\right) \rightarrow\left(\mathcal{O}_{K} / \pi^{n} \mathcal{O}_{K}\right)^{*}
$$

which is the map induced by the representation $\tau: G_{K} \rightarrow \mathcal{O}_{K}^{*}$ (cf. $\S 7.1$, equation (7.5) ), is an isomorphism since $\left[K_{n}\left\{\left\{T_{1}\right\}\right\} \cdots\left\{\left\{T_{d-1}\right\}\right\} / K\left\{\left\{T_{1}\right\}\right\} \cdots\left\{\left\{T_{d-1}\right\}\right\}\right]=$ $\left[K_{n}: K\right]=q^{n}-q^{n-1},\left|\left(\mathcal{O}_{K} / \pi^{n} \mathcal{O}_{K}\right)^{*}\right|=q^{n}-q^{n-1}$.

Proposition 8.0.1. Let $w_{n}=l^{\prime}\left(e_{n}\right) d e_{n}, n \geq 1$. Then $w_{n}$ generate $\Omega_{C}\left(\mathcal{O}_{\bar{K}}\right)$ as $\mathcal{O}_{\bar{K}}$-modules. We also have

$$
w_{n}=\pi w_{n+1} .
$$

Proof. cf. [14] Proposition 7.9.

Proposition 8.0.2. $w_{n}^{g}=\tau(g) w_{n}$ for all $g \in G(\bar{K} / K)$.

Proof. cf. [14] § 7.2.3.

Let $M$ be a finite extension of $K_{s}, \pi_{M}$ a uniformizer for $M$ and let $\mathcal{M}$ denote the field $M\left\{\left\{T_{1}\right\}\right\} \cdots\left\{\left\{T_{d-1}\right\}\right\}$. Let $\pi_{1}$ be a uniformizer for $K_{1}=K\left(\kappa_{1}\right)$. Let

$$
P_{\mathcal{M}}=\left(1 /\left(\pi_{1} D(M / K)\right)\right) \mathcal{O}_{\mathcal{M}}=\left\{x \in \mathcal{M}: v_{\mathcal{M}}(x) \geq-v_{\mathcal{M}}\left(\pi_{1} D(M / K)\right)\right\}
$$


We define a $d$-dimensional derivation

$$
Q_{\mathcal{M}, s}: \mathcal{O}_{\mathcal{M}}^{d} \rightarrow P_{\mathcal{M}} /\left(\pi^{s} / \pi_{1}\right) P_{\mathcal{M}}
$$

over $\mathcal{O}_{K}$, in the following way. Let $b^{\prime} \in \mathcal{O}_{M}$ such that $w_{s}=l^{\prime}\left(e_{s}\right) d e_{s}=b^{\prime} d \pi_{M}$, then $b^{\prime} \mathcal{O}_{M}=D\left(M / K_{s}\right)$. Let us put

$$
Q_{\mathcal{M}, s}\left(T_{1}, \ldots, T_{d-1}, \pi_{M}\right)=\frac{T_{1} \cdots T_{d-1}}{b^{\prime} \pi^{s}}
$$

Clearly, $T_{1} \cdots T_{d-1} /\left(b^{\prime} \pi^{s}\right) \in P_{\mathcal{M}}$. By Proposition 6.1.5 (2) the period of $d e_{s}$ is generated by $\pi^{s} / \pi_{1}$, then $D\left(K_{s} / K\right)=\pi^{s} / \pi_{1} \mathcal{O}_{s}$ and so

$$
D(M / K)=D\left(M / K_{s}\right) D\left(K_{s} / K\right)=D\left(M / K_{s}\right)\left(\pi^{s} / \pi_{1}\right) .
$$

Hence $D(M / K) Q_{\mathcal{M}, s}\left(T_{1}, \ldots, T_{d-1}, \pi_{M}\right) \in\left(\pi^{s} / \pi_{1}\right) P_{\mathcal{M}}$. Therefore, by Proposition 6.2.3. $Q_{\mathcal{M}, s}$ defines a $d$-dimensional derivation as follows

$$
Q_{\mathcal{M}, s}\left(\alpha_{1}, \ldots, \alpha_{d}\right):=\operatorname{det}\left[\frac{\partial \alpha_{i}}{\partial T_{j}}\right]_{1 \leq i, j \leq d} \frac{T_{1} \cdots T_{d-1}}{b^{\prime} \pi^{s}}
$$

where $\alpha_{1}, \ldots, \alpha_{d} \in \mathcal{O}_{\mathcal{M}}$. Note that the definition of $Q_{\mathcal{M}, s}$ is independent of the choice of uniformizer $\pi_{M}$ of $M$.

Proposition 8.0.3. Let $M / L / K_{s}, M \supset K_{t}$, be a finite tower of local fields and let

$$
\mathcal{L}=L\left\{\left\{T_{1}\right\}\right\} \cdots\left\{\left\{T_{d-1}\right\}\right\} \quad \text { and } \quad \mathcal{M}=M\left\{\left\{T_{1}\right\}\right\} \cdots\left\{\left\{T_{d-1}\right\}\right\}
$$

Suppose that $D(M / L) \mid \pi^{t-s}$. Then $\left(\pi^{t} / \pi_{1}\right) P_{\mathcal{M}}$ is contained in $X=\left(\pi^{s} / \pi_{1}\right) P_{\mathcal{L}} \mathcal{O}_{\mathcal{M}}$ so 
$Q_{\mathcal{M}, t}(\bmod X)$ is well-defined. Let

$$
\alpha: P_{\mathcal{L}} /\left(\pi^{s} / \pi_{1}\right) P_{\mathcal{L}} \rightarrow P_{\mathcal{M}} / X
$$

be the injection induced by the composition $P_{\mathcal{L}} \subset P_{\mathcal{M}}$. Then for $y \in \mathcal{O}_{\mathcal{L}}^{d}$

$$
\alpha\left(Q_{\mathcal{L}, s}(y)\right)=Q_{\mathcal{M}, t}(y) \quad(\bmod X) .
$$

Proof. The Proposition 8.0 .3 and its proof were suggested by Professor V. Kolyvagin.

First,

$\frac{\pi^{t}}{\pi_{1}} P_{\mathcal{M}}=\frac{\pi^{t}}{\pi_{1}} \frac{1}{\pi_{1} D(M / K)}=\frac{\pi^{t}}{\pi_{1}} \cdot \frac{1}{\pi_{1} D(M / L) D(L / K)}=\frac{\pi^{t}}{\pi_{1}} \cdot \frac{\pi^{t-s}}{D(M / L)} \cdot \frac{1}{\pi_{1} D(L / K)} \subset X$

because $\left(\pi^{t-s} / D(M / L)\right) \subset \mathcal{O}_{\mathcal{M}}$ by our assumption.

Let $a$ and $c$ in $\mathcal{O}_{M}$ and $b \in \mathcal{O}_{L}$ are such that

$$
w_{t}=c d \pi_{M}, w_{s}=b d \pi_{L} \text { and } \quad d \pi_{L}=a d \pi_{M} .
$$

Because $\pi^{t-s} w_{t}=w_{s}$ we have $\pi^{t-s} c d \pi_{M}=b d \pi_{M}=b a d \pi_{M}$. So

$$
\pi^{t-s} c \equiv b a \quad(\bmod D(M / L)) .
$$

Dividing this congruence by $\pi^{t} c b$ and taking into account that $c \mathcal{O}_{M}=D\left(M / K_{t}\right)$ and $b \mathcal{O}_{L}=D\left(L / K_{s}\right)$, we have

$$
\frac{1}{\pi^{s} b} \equiv \frac{a}{\pi^{t} c} \quad(\bmod Z)
$$


where

$$
Z=\frac{D(M / K)}{\pi^{t} D\left(M / K_{t}\right) D\left(L / K_{s}\right)}=\frac{\mathcal{O}_{\mathcal{M}}}{\pi_{1} D\left(L / K_{s}\right)}=\frac{\pi^{s}}{\pi_{1}} \frac{\mathcal{O}_{\mathcal{M}}}{\pi_{1} D(L / K)}=X
$$

(we are using that $D(M / K)=D\left(M / K_{t}\right) D\left(K_{t} / K\right)$ and $D\left(K_{t} / K\right)=\pi^{t} / \pi_{1}, D(L / K)=$ $\left.\left(\pi^{s} / \pi_{1}\right) D\left(L / K_{s}\right)\right)$. So

$$
Q_{\mathcal{M}, t}\left(T_{1}, \ldots, T_{d-1}, \pi_{L}\right)=\frac{a}{\pi^{t} c}=\frac{1}{\pi^{s} b} \quad(\bmod X)=\alpha\left(Q_{\mathcal{L}, s}\left(T_{1}, \ldots, T_{d-1}, \pi_{L}\right)\right) .
$$

This implies the corresponding equality for arbitrary $y \in \mathcal{O}_{\mathcal{L}}^{d}$ since right hand and left hand mappings are multidimensional derivations of $\mathcal{O}_{\mathcal{L}}^{d}$ over $\mathcal{O}_{K}$.

Proposition 8.0.4. $Q_{\mathcal{M}, s}^{g}=\tau\left(g^{-1}\right) Q_{\mathcal{M}, s}$. Here $Q_{\mathcal{M}, s}^{g}$ is the d-dimensional derivation defined by $Q_{\mathcal{M}, s}^{g}\left(a_{1}, \ldots, a_{d-1}\right)=\left[Q_{\mathcal{M}, s}\left(a_{1}^{g^{-1}}, \ldots, a_{d}^{g^{-1}}\right)\right]^{g}$.

Proof. Notice that it is enough to check that

$$
Q_{\mathcal{M}, s}^{g}\left(T_{1}, \ldots, T_{d-1}, \pi_{M}\right)=\tau\left(g^{-1}\right) Q_{\mathcal{M}, s}\left(T_{1}, \ldots, T_{d-1}, \pi_{M}\right),
$$

by equation 6.6. of Proposition 6.2.3.

Let $b^{\prime} \in \mathcal{O}_{M}$ such that $w_{s}=b^{\prime} d \pi_{M}$. Then $w_{s}^{g}=\left(b^{\prime}\right)^{g} d \pi_{M}^{g}$ and, by Proposition 8.0.2, we have that $w_{s}=\tau\left(g^{-1}\right)\left(b^{\prime}\right)^{g} d \pi_{M}^{g}$. Since $\pi_{M}^{g}$ is also a uniformizer for $M$ and the definition of $Q_{\mathcal{M}, s}$ is independent of the uniformizer, then

$$
Q_{\mathcal{M}, s}\left(T_{1}, \ldots, T_{d-1}, \pi_{M}^{g}\right)=\frac{1}{\tau\left(g^{-1}\right)\left(b^{\prime}\right)^{g} \pi^{s}},
$$


but

$$
\frac{1}{\tau\left(g^{-1}\right)\left(b^{\prime}\right)^{g} \pi^{s}}=\tau(g)\left(\frac{1}{b^{\prime} \pi^{s}}\right)^{g}
$$

This last expression is equal to $\tau(g) Q_{M, s}\left(T_{1}, \ldots, T_{d-1}, \pi_{M}\right)^{g}$ by equation 8.3 .

Proposition 8.0.5. Let $M$ be a finite extension of $K, M \cap K_{\infty}=K_{s}$ and let $N=M_{s+1}$. Put $\mathcal{N}=N\left\{\left\{T_{1}\right\}\right\} \cdots\left\{\left\{T_{d-1}\right\}\right\}$ and $\mathcal{M}=M\left\{\left\{T_{1}\right\}\right\} \cdots\left\{\left\{T_{d-1}\right\}\right\}$. Then

$$
\tau_{s+1}(G(\mathcal{N} / \mathcal{M}))=\frac{1+\pi^{s} C}{1+\pi^{s+1} C} \subset\left(C / \pi^{s+1} C\right)^{*}
$$

where $C=\mathcal{O}_{K}$, and the element

$$
\sum_{g \in G(\mathcal{N} / \mathcal{M})}\left(\tau_{s+1}(g)-1\right) g
$$

takes $(\pi / D(N / M)) \mathcal{O}_{\mathcal{N}}$ to $\left(\pi^{s+1} / \pi_{1}\right) \mathcal{O}_{\mathcal{N}}$. Also $D(N / M) \mid \pi$.

Proof. This follows immediately from the fact that $G(N / M) \cong G(\mathcal{N} / \mathcal{M})$ and Proposition 5.12 in [14] and its proof.

Let $M$ be a finite extension of $K_{s}$ and $\mathcal{M}=M\left\{\left\{T_{1}\right\}\right\} \cdots\left\{\left\{T_{d-1}\right\}\right\}$. From $Q_{M, s}$ we can define the logarithmic derivative 


$$
Q L_{\mathcal{M}, s}: K_{d}(\mathcal{M}) \rightarrow \frac{\frac{1}{\pi_{M}} P_{\mathcal{M}}}{\frac{\pi^{s}}{\pi_{1} \pi_{M}} P_{\mathcal{M}}}
$$

by

$$
\left\{\begin{aligned}
Q L_{\mathcal{M}, s}\left(u_{1}, \ldots, u_{d-1}, \pi_{M}\right) & =\frac{Q_{\mathcal{M}, s}\left(u_{1}, \ldots, u_{d-1}, \pi_{M}\right)}{u_{1} \cdots u_{d-1} \pi_{M}}\left(\bmod \frac{\pi^{s}}{\pi_{1} \pi_{M}} P_{\mathcal{M}}\right), \\
Q L_{\mathcal{M}, s}\left(u_{1}, \ldots, u_{d}\right) & =\frac{Q_{\mathcal{M}, s}\left(u_{1}, \ldots, u_{d}\right)}{u_{1} \cdots u_{d}}\left(\bmod \frac{\pi^{s}}{\pi_{1}} P_{\mathcal{M}}\right) \\
Q L_{\mathcal{M}, s}\left(u_{1}, \ldots, \pi_{M}^{k} u_{d}\right) & =k Q L_{\mathcal{M}, s}\left(u_{1}, \ldots, \pi_{M}\right)+Q L_{\mathcal{M}, s}\left(u_{1}, \ldots, u_{d}\right), k \in \mathbb{Z} \\
Q L_{\mathcal{M}, s}\left(a_{1}, \ldots, a_{d-1}\right) & =0, \text { whenever } a_{i}=a_{j} \text { for } i \neq j \text { and } a_{1}, \ldots, a_{d-1} \in \mathcal{M}^{*}
\end{aligned}\right.
$$

where $u_{1}, \ldots, u_{d}$ are in $\mathcal{O}_{\mathcal{M}}^{*}=\left\{x \in \mathcal{O}_{\mathcal{M}}: v_{\mathcal{M}}(x)=0\right\}$. Notice the forth property says that $Q L_{\mathcal{M}, s}$ is alternate, in particular it is skew-symmetric, i.e,

$$
Q L_{\mathcal{M}, s}\left(a_{1}, \ldots, a_{i}, \ldots, a_{j}, \ldots, a_{d}\right)=-Q L_{\mathcal{M}, s}\left(a_{1}, \ldots, a_{j}, \ldots, a_{i}, \ldots, a_{d}\right) .
$$

whenever $i \neq j$.

Let $L \supset K$ and take the smallest $r$ such that $L \cap K_{\pi} \subset K_{r}$. Let $\gamma_{m}$ be a uniformizer for $L_{m}=L\left(\kappa_{m}\right)$ and define $\mathcal{L}_{m}=L_{m}\left\{\left\{T_{1}\right\}\right\} \cdots\left\{\left\{T_{d-1}\right\}\right\}$. Then

$$
L_{s} \cap K_{\pi}=\left(L \cap K_{\pi}\right) K_{s}=K_{s} \quad(s \geq r) .
$$

Let $\mathcal{M}=\mathcal{L}_{s}$. For abbreviation we will write $P_{s}, Q_{s}, Q L_{s}, N_{t / s}$ and $\operatorname{Tr}_{t / s}$ instead of 
$P_{\mathcal{L}_{s}}, Q_{\mathcal{M}, s}, Q L_{\mathcal{M}, s}, N_{\mathcal{L}_{s} / \mathcal{L}_{t}}$ and $\operatorname{Tr}_{\mathcal{L}_{s} / \mathcal{L}_{t}}$. Moreover

$$
\operatorname{Tr}_{t / s}\left(P_{t}\right) \subset P_{s}
$$

and

$$
\operatorname{Tr}_{t / s}\left(\left(1 / \gamma_{t}\right) P_{t}\right) \subset\left(1 / \gamma_{s}\right) P_{s}
$$

Indeed, since $D\left(L_{t} / K\right)=D\left(L_{t} / L_{s}\right) D\left(L_{s} / K\right)$ then $P_{t}=\left(1 / D\left(L_{t} / L_{s}\right)\right) P_{s}$ and so

$$
\operatorname{Tr}_{t / s}\left(P_{t}\right)=\operatorname{Tr}_{t / s}\left(\left(1 / D\left(L_{t} / L_{s}\right)\right) P_{s}\right)=P_{s} \operatorname{Tr}_{t / s}\left(1 / D\left(L_{t} / L_{s}\right)\right) \subset P_{s},
$$

and $\operatorname{Tr}_{t / s}\left(\left(\gamma_{s} / \gamma_{t}\right) P_{t}\right) \subset \operatorname{Tr}_{t / s}\left(P_{t}\right) \subset P_{s}$.

Proposition 8.0.6. For $s \geq r+1$ and $t \geq s$ we have

$$
\begin{gathered}
Q L_{s}\left(N_{t / s}\left(a_{1}\right), a_{2}, \ldots, a_{d}\right)=\operatorname{Tr}_{t / s}\left(Q L_{t}\left(a_{1}, \ldots, a_{d}\right)\right) \quad\left(\in \frac{\left(1 / \gamma_{s}\right) P_{s}}{\left(\pi^{s} / \pi_{1} \gamma_{s}\right) P_{s}}\right) \\
\text { for } a_{1} \in \mathcal{L}_{t}^{*}=L_{t}\left\{\left\{T_{1}\right\}\right\} \cdots\left\{\left\{T_{d-1}\right\}\right\}^{*} \text { and } a_{2}, \ldots, a_{d} \in \mathcal{L}_{s}^{*}=L_{s}\left\{\left\{T_{1}\right\}\right\} \cdots\left\{\left\{T_{d-1}\right\}\right\}^{*} .
\end{gathered}
$$

Proof. It will be enough to consider the case $t=s+1$. From Proposition 8.0.4 it follows that 


$$
\begin{aligned}
Q L_{t}\left(\prod_{g \in G\left(\mathcal{L}_{t} / \mathcal{L}_{s}\right)} a_{1}^{g}, a_{2}, \ldots, a_{d}\right)= & \left(\sum \tau_{t}(g) g\right) Q L_{t}\left(a_{1}, \ldots, a_{d}\right) \\
= & \left(\sum g\right) Q L_{t}\left(a_{1}, \ldots, a_{d}\right) \\
& +\left(\sum\left(\tau_{t}(g)-1\right) g\right) Q L_{t}\left(a_{1}, \ldots, a_{d}\right)
\end{aligned}
$$

and by Proposition 8.0 .5 we see that $\sum\left(\delta_{t}(g)-1\right) g$ takes

$$
P_{t}=\frac{1}{D\left(L_{t} / L_{s}\right)} P_{s}=\frac{\pi}{D\left(L_{t} / L_{s}\right)}\left(\frac{1}{\pi} P_{s}\right)
$$

to

$$
\left(\frac{\pi^{t}}{\pi_{1}} \mathcal{O}_{\mathcal{L}_{t}}\right)\left(\frac{1}{\pi} P_{s}\right)=\frac{\pi^{s}}{\pi_{1}} P_{s} \mathcal{O}_{\mathcal{L}_{t}}
$$

Then

$$
\begin{aligned}
Q L_{s}\left(N_{t / s}\left(a_{1}\right), a_{2}, \ldots, a_{d}\right) & =Q L_{t}\left(N_{t / s}\left(a_{1}\right), a_{2}, \ldots, a_{d}\right) \quad\left(\bmod \frac{\pi^{s}}{\pi_{1} \gamma_{s}} P_{s}\right) \\
& =\operatorname{Tr}_{t / s}\left(Q L_{t}\left(a_{1}, \ldots, a_{d}\right)\right)
\end{aligned}
$$

where the first equality follows from Proposition 8.0.3. 


\subsection{Computations of the invariants}

We will assume that $p \neq 2$. Let $(m, t)$ be an admissible pair, $M=K_{t}$ and $\mathcal{M}=$ $M\left\{\left\{T_{1}\right\}\right\} \cdots\left\{\left\{T_{d-1}\right\}\right\}$. We can take $e_{t}$ as a uniformizer $\pi_{t}$ of $K_{t}$. We will show that

$$
\begin{aligned}
\left(\left\{T_{1}, \ldots, T_{d-1}, u\right\}, e_{t}\right)_{\mathcal{M}, m} & =\mathbb{T}_{\mathcal{M} / K}\left(\log u \cdot\left(-\frac{1}{\pi^{t}}\right)\right) \quad\left(\bmod \pi^{n} C\right) \\
& =\operatorname{Tr}_{K_{t} / K}\left(c_{\mathcal{M} / K_{t}}(\log u) \cdot\left(-\frac{1}{\pi^{t}}\right)\right) \quad\left(\bmod \pi^{n} C\right)
\end{aligned}
$$

for all $u \in V_{\mathcal{M}, 1}=1+\mu_{\mathcal{M}, 1}$. From equation 7.7$)$ we have

$$
c_{\beta}=-\frac{1}{\pi^{t}} \quad\left(\bmod \pi^{m} R_{t, 1}\right) .
$$

Furthermore, every $u \in V_{\mathcal{M}, 1}$ can be expressed as

$$
\prod_{\substack{\bar{i}=\left(i_{1}, \ldots, i_{d}\right) \in S \\ i_{d} \geq\left[v_{M}(p) /(p-1)\right]+1}}\left(1+\theta_{\bar{i}} T_{1}^{i_{1}} \cdots T_{d-1}^{i_{d-1}} \pi_{t}^{i_{d}}\right)
$$

where $\theta_{i} \in \mathcal{R}, \mathcal{R}$ is the group of $q-1$ th roots of 1 in $K_{t}^{*}$, and $S \subset \mathbb{Z}^{d}$ is an admissible set ( see Corollary from Section 1.4.3 [7]), then it is enough to check (8.11) for

$$
u=1+\theta T_{1}^{i_{1}} \cdots T_{d-1}^{i_{d-1}} \pi_{t}^{i_{d}}, \quad\left(\theta^{q-1}=1\right) .
$$

Case 1) Suppose $\left(i_{1} \ldots, i_{d-1}\right) \neq(0, \ldots, 0)$. Then the right hand side of 8.11) is zero since $c_{\mathcal{M} / K_{t}}(\log u)=0$. Let us show that the left hand side is also zero as well.

Suppose first $i_{k}>0$ for some $1 \leq k \leq d-1$. Consider $\mathcal{N}=K_{t}\left\{\left\{Y_{1}\right\}\right\} \ldots\left\{\left\{Y_{d-1}\right\}\right\}$ where $Y_{k}=T_{k}^{i_{k}}$ and $Y_{r}=T_{r}$, for $r \neq k$. By lemma 8.1.1 below, $\mathcal{M} / \mathcal{N}$ is a finite 
extension of degree $i_{k}$ and $N_{\mathcal{M} / \mathcal{N}}\left(T_{k}\right)= \pm Y_{k}$. Let also $i_{k}^{\prime}=1$ and $i_{r}^{\prime}=i_{r}$ for $r \neq k$.

Therefore by proposition 4.2.1 (4)

$$
\begin{aligned}
& \left(\left\{T_{1}, \ldots, T_{d-1}, 1+\theta_{i} T_{1}^{i_{1}} \cdots T_{d-1}^{i_{d-1}} \pi_{t}^{i_{d}}\right\}, e_{t}\right)_{\mathcal{M}, m}= \\
= & \left(N_{\mathcal{M} / \mathcal{N}}\left\{T_{1}, \ldots, T_{d-1}, 1+\theta_{i} T_{1}^{i_{1}} \cdots T_{d-1}^{i_{d-1}} \pi_{t}^{i_{d}}\right\}, e_{t}\right)_{\mathcal{N}, m} \\
= & \left(\left\{Y_{1}, \ldots, N_{\mathcal{M} / \mathcal{N}}\left(T_{k}\right), \ldots, Y_{d-1}, 1+\theta Y_{1}^{i_{1}^{\prime}} \cdots Y_{d-1}^{i_{d-1}^{\prime}} \pi_{t}^{i_{d}}\right\}, e_{t}\right)_{\mathcal{N}, m} \\
= & \left(\left\{Y_{1}, \ldots, \pm 1, \ldots, Y_{d-1}, 1+\theta Y_{1}^{i_{1}^{\prime}} \cdots Y_{d-1}^{i_{d-1}^{\prime}} \pi_{t}^{i_{d}}\right\}, e_{t}\right)_{\mathcal{N}, m} \\
& \oplus\left(\left\{Y_{1}, \ldots, Y_{d-1}, 1+\theta Y_{1}^{i_{1}^{\prime}} \cdots Y_{d-1}^{i_{d-1}^{\prime}} \pi_{t}^{i_{d}}\right\}, e_{t}\right)_{\mathcal{N}, m}
\end{aligned}
$$

Since $p \neq 2,\left(\left\{Y_{1}, \ldots, \pm 1, \ldots, Y_{d-1}, 1+\theta Y_{1}^{i_{1}^{\prime}} \cdots Y_{d-1}^{i_{d-1}^{\prime}} \pi_{t}^{i_{d}}\right\}, e_{t}\right)_{\mathcal{N}, m}=0 . \quad$ On the other hand, since $\theta^{q-1}=1$ then

$$
\begin{aligned}
& \left(\left\{Y_{1}, \ldots, \theta, \ldots, Y_{d-1}, 1+\theta Y_{1}^{i_{1}^{\prime}} \cdots Y_{d-1}^{i_{d-1}^{\prime}} \pi_{t}^{i_{d}}\right\}, e_{t}\right)_{\mathcal{N}, m} \\
& =[1 /(q-1)]\left(\left\{Y_{1}, \ldots, \theta^{q-1}, \ldots, Y_{d-1}, 1+\theta Y_{1}^{i_{1}^{\prime}} \cdots Y_{d-1}^{i_{d-1}^{\prime}} \pi_{t}^{i_{d}}\right\}, e_{t}\right)_{\mathcal{N}, m}=0
\end{aligned}
$$

and also $\left(\left\{Y_{1}, \ldots, \pi_{t}, \ldots, Y_{d-1}, 1+\theta Y_{1}^{i_{1}^{\prime}} \cdots Y_{d-1}^{i_{d-1}^{\prime}} \pi_{t}^{i_{d}}\right\}, e_{t}\right)_{\mathcal{N}, m}=0$ by the norm series relation for Lubin-Tate formal groups $\left(\left\{a_{1}, \ldots,-X, \ldots, a_{d}\right\}, X\right)_{\mathcal{N}, m}=0$ and 
recalling that $\pi_{t}=e_{t}$. Thus

$$
\begin{aligned}
& \left(\left\{T_{1}, \ldots, T_{d-1}, 1+\theta T_{1}^{i_{1}} \cdots T_{d-1}^{i_{d-1}} \pi_{t}^{i_{d}}\right\}, e_{t}\right)_{\mathcal{M}, m} \\
= & \left(\left\{Y_{1}, \ldots, Y_{k}, \ldots, Y_{d-1}, 1+\theta Y_{1}^{i_{1}^{\prime}} \cdots Y_{d-1}^{i_{d-1}^{\prime}} \pi_{t}^{i_{d}}\right\}, e_{t}\right)_{\mathcal{N}, m} \\
= & \left(\left\{Y_{1}, \ldots, \theta Y_{1}^{i_{1}^{\prime}} \cdots Y_{k}^{i_{i}^{\prime}} \cdots Y_{d-1}^{i_{d-1}^{\prime}} \pi_{t}^{i_{d}}, \ldots, Y_{d-1}, 1+\theta Y_{1}^{i_{1}^{\prime}} \cdots Y_{d-1}^{i_{d-1}^{\prime}} \pi_{t}^{i_{d}}\right\}, e_{t}\right)_{\mathcal{N}, m} \\
= & \left(\left\{Y_{1}, \ldots,-\theta Y_{1}^{i_{1}^{\prime}} \cdots Y_{d-1}^{i_{d}^{\prime}} \pi_{t}^{i_{d}}, \ldots, Y_{d-1}, 1+\theta Y_{1}^{i_{1}^{\prime}} \cdots Y_{d-1}^{i_{d-1}^{\prime}} \pi_{t}^{i_{d}}\right\}, e_{t}\right)_{\mathcal{N}, m}
\end{aligned}
$$

The second equality follows from the fact that $\left\{Y_{1}, \ldots, Y_{r}, \ldots, Y_{d-1}, 1+\theta Y_{1}^{i_{1}^{\prime}} \cdots Y_{d-1}^{i_{d-1}^{\prime}} \pi_{t}^{i_{d}}\right\}$ is trivial, for $r \neq k$, in the Milnor K-group $K_{d}(\mathcal{M})$. Moreover, the last expression in the chain of equalities is again zero because $\left\{Y_{1}, \ldots,-\theta Y_{1}^{i_{1}^{\prime}} \ldots Y_{d-1}^{i_{d-1}^{\prime}} \pi_{t}^{i_{d}}, \ldots, Y_{d-1}, 1+\right.$ $\left.\theta Y_{1}^{i_{1}^{\prime}} \cdots Y_{d-1}^{i_{d-1}^{\prime}} \pi_{t}^{i_{d}}\right\}$ is the zero element, by the Steinberg property, in the Milnor Kgroup $K_{d}(\mathcal{M})$.

Suppose now $i_{k}<0$. We take $Y_{k}=T_{k}^{-i_{k}}$ instead and by lemma 8.1.1 we have $N_{\mathcal{M} / \mathcal{N}}\left(T_{k}^{-1}\right)= \pm T_{k}^{-i_{k}}= \pm Y_{k}$. Noticing that

$$
\begin{aligned}
& \left(\left\{T_{1}, \ldots, T_{k}, \ldots, T_{d-1}, 1+\theta T_{1}^{i_{1}} \cdots T_{d-1}^{i_{d-1}} \pi_{t}^{i_{d}}\right\}, e_{t}\right)_{\mathcal{M}, m} \\
& =-\left(\left\{T_{1}, \ldots, T_{k}^{-1}, \ldots, T_{d-1}, 1+\theta T_{1}^{i_{1}} \cdots T_{d-1}^{i_{d-1}} \pi_{t}^{i_{d}}\right\}, e_{t}\right)_{\mathcal{M}, m}
\end{aligned}
$$

we can now apply the same argument as before to conclude that

$$
\left(\left\{T_{1}, \ldots, T_{k}, \ldots, T_{d-1}, 1+\theta T_{1}^{i_{1}} \cdots T_{d-1}^{i_{d-1}} \pi_{t}^{i_{d}}\right\}, e_{t}\right)_{\mathcal{M}, m}=0
$$

Case 2) Suppose $\left(i_{1} \ldots, i_{d-1}\right)=(0, \ldots, 0)$. That is, when $u$ is an element of the 
one dimensional local field $K_{t}$. In this case, we will show in lemma 8.1 .2 below that the pairing $\left(\left\{T_{1} \ldots, T_{d-1}, u\right\}, e_{t}\right)_{\mathcal{M}, m}$ coincides with the pairing taking values in the one dimensional local field $K_{t}:\left(u, e_{t}\right)_{K_{t}, m}$. Thus, by [14] section 7.3.1 and the fact that $c_{\mathcal{M} / K}(\log (u))=\log (u)$ formula 8.11 follows.

Lemma 8.1.1. Let $M$ be a complete discrete valuation field and $\mathcal{M}=M\{\{T\}\}$. Put $Y=T^{j}$ for $j>0$. Define $\mathcal{N}=M\{\{Y\}\}$. Then $\mathcal{M} / \mathcal{N}$ is a finite extension of degree $j$ and $N_{\mathcal{M} / \mathcal{N}}(T)= \pm Y$.

Remark: Since $M$ is a complete discrete valuation field, the result immedeately generalizes to the $d$-dimensional case, for if $\mathcal{L}=L\left\{\left\{T_{1}\right\}\right\} \cdots\left\{\left\{T_{d-1}\right\}\right\}$, then we can take $M$ to be $L\left\{\left\{T_{1}\right\}\right\} \ldots\left\{\left\{T_{d-2}\right\}\right\}$ and apply the result to $\mathcal{M}=M\left\{\left\{T_{d-1}\right\}\right\}$ and $\mathcal{N}=M\left\{\left\{Y_{d-1}\right\}\right\}$, where $Y_{d-1}=T_{d-1}^{j}$.

Proof. We can assume that $M$ contains $\zeta_{j}$, a primitive $j$ th root of unity of 1 . Otherwise we can considering the diagram

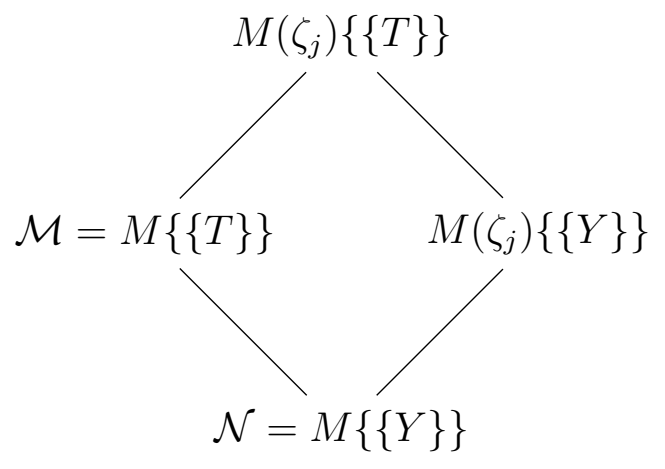

we see that $[\mathcal{M} / \mathcal{N}]=\left[M\left(\zeta_{j}\right)\{\{T\}\}: M\left(\zeta_{j}\right)\{\{Y\}\}\right]$ since $M\{\{T\}\} \cap\left(M\left(e_{j}\right)\{\{T\}\}\right)=$ 
$M\{\{Y\}\}$

Note that $Y$ has exact order $j$ in $\mathcal{N}^{*} /\left(\mathcal{N}^{*}\right)^{j}$ for if $Y=\alpha^{k}, \alpha \in \mathcal{N}^{*}$, then $0=$ $v_{\mathcal{N}}(Y)=k v_{\mathcal{N}}(\alpha)$, thus $\alpha \in \mathcal{O}_{\mathcal{N}}^{*}$, and we can go to the residue field $k_{\mathcal{N}}$ of $\mathcal{N}$ where we have $1=v_{k_{\mathcal{N}}}(\bar{Y})=k v_{k_{\mathcal{N}}}(\bar{\alpha})$, which implies $k=1$. Then by Kummer theory (cf. [2] Chapter 3 Lemma 2) we have that the polynomial $P(X)=X^{j}-Y \in \mathcal{O}_{\mathcal{N}}[X]$ is irreducible. Thus $[\mathcal{M} / \mathcal{N}]=j$, and $N_{\mathcal{M} / \mathcal{N}}(T)$ is the product of the roots of the polynomial $P(X)$. These roots are $\zeta_{j}^{k} T, k=1, \ldots, j$. Thus

$$
N_{\mathcal{M} / \mathcal{N}}(T)=\prod_{k=1}^{j} \zeta_{j}^{k} T=\zeta_{j}^{\frac{j(j+1)}{2}} T^{j}=\left\{\begin{array}{ll}
T^{j}=Y, & \text { if } j \text { is odd }, \\
-T^{j}=-Y, & \text { if } j \text { is even. }
\end{array}=(-1)^{j+1} Y .\right.
$$

Lemma 8.1.2. For a local field $L / \mathbb{Q}_{p}$, let $\mathcal{L}=L\left\{\left\{T_{1}\right\}\right\} \cdots\left\{\left\{T_{d-1}\right\}\right\}$ and consider the map

$$
f: L^{*} \rightarrow K_{d}(\mathcal{L}) \rightarrow \operatorname{Gal}\left(\mathcal{L}^{a b} / \mathcal{L}\right) \rightarrow \operatorname{Gal}\left(L^{a b} / L\right)
$$

defined by

$$
\left.a \rightarrow\left\{T_{1}, \ldots, T_{d-1}, a\right\} \rightarrow \Upsilon_{\mathcal{L}}\left(\left\{T_{1}, \ldots, T_{d-1}, a\right\}\right) \rightarrow \Upsilon_{\mathcal{L}}\left(\left\{T_{1}, \ldots, T_{d-1}, a\right\}\right)\right|_{G a l\left(L^{a b} / L\right)}
$$

This coincides with the reciprocity map for $L, \theta_{L}: L^{*} \rightarrow \operatorname{Gal}\left(L^{a b} / L\right)$. Thus, for $L=K_{t}$ and $\mathcal{M}=K_{t}\left\{\left\{T_{1}\right\}\right\} \cdots\left\{\left\{T_{d-1}\right\}\right\}$ we have

$$
\left(\left\{T_{1}, \ldots, T_{d-1}, u\right\}, e_{t}\right)_{\mathcal{M}, m}=\left(u, e_{t}\right)_{K_{t}, m}
$$


for all $u \in V_{L, 1}=\left\{x \in L: v_{L}(x-1)>\left[v_{L}(p) /(p-1)\right]+1\right\}$.

Proof. It is enough to verify the two conditions of [2] Chapter $5 \S 2.8$ Proposition 6 . Let $\mathcal{L}_{d}$ be $\mathcal{L}$, and $\mathcal{L}_{d-1}=k_{\mathcal{L}\left(\left(t_{1}\right)\right) \cdots\left(\left(t_{d-1}\right)\right)}, \ldots, \mathcal{L}_{0}=k_{\mathcal{L}}$ the chain of residue fields of $\mathcal{L}$. By (2) of Theorem 4.1.1 we have

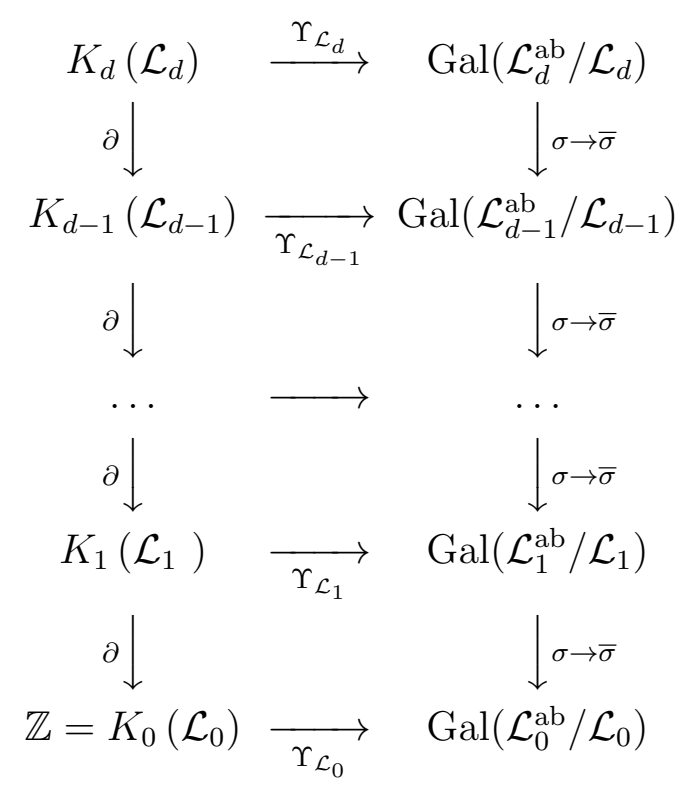

By Remark 4.1 .3 the composition of the vertical maps $\partial$ 's we have

$$
\partial\left(\partial\left\{T_{1} \ldots, T_{d-1}, a\right\}\right)=v_{L}(a),
$$

thus $f: L^{*} \rightarrow \operatorname{Gal}\left(L^{a b} / L\right) \rightarrow \operatorname{Gal}\left(L^{u n} / L\right)$ is the valuation map $v_{L}: L^{*} \rightarrow \mathbb{Z}$. Thus condition (1) of [2] Chapter $5 \S 2.8$ Proposition 6 is verified.

If $a \in L^{*}, L^{\prime} / L$ is a finite abelian extension, $\mathcal{L}^{\prime}=L^{\prime}\left\{\left\{T_{1}\right\}\right\} \cdots\left\{\left\{T_{d-1}\right\}\right\}, \mathcal{L}=$ $L\left\{\left\{T_{1}\right\}\right\} \cdots\left\{\left\{T_{d-1}\right\}\right\}$, and $a$ is a norm from $L^{\prime *}$, namely $a=N_{L^{\prime} / L}(\alpha)$, then clearly 
$\left\{T_{1}, \ldots, T_{d-1}, a\right\}$ is a norm from $K_{d}\left(\mathcal{L}^{\prime}\right)$, namely

$$
\left\{T_{1}, \ldots, T_{d-1}, a\right\}=\left\{T_{1}, \ldots, T_{d-1}, N_{L^{\prime} / L}(\alpha)\right\}=N_{\mathcal{L}^{\prime} / \mathcal{L}}\left\{T_{1}, \ldots, T_{d-1}, \alpha\right\},
$$

and by (1) of Theorem 4.1.1 we have that $\Upsilon_{L\left\{\left\{T_{1}\right\}\right\} \cdots\left\{\left\{T_{d-1}\right\}\right\}}\left(T_{1}, \ldots, T_{d-1}, a\right)$ is trivial on $\mathcal{L}^{\prime}$ and so $f(a)$ is trivial on $L^{\prime}$. Thus condition (2) of [2] Chapter $5 \S 2.8$ Proposition 6 is verified.

\subsection{Explicit formulas for the Lubin-Tate formal groups}

In this subsection we will provide a refinement of the formulas given for Theorem 7.3.1 to the case of a Lubin-Tate formal group $F_{f}$.

Let us introduce the following simplified notation throughout this subsection. Let $L$ be a finite extension of $K_{n}$ and put $\mathcal{L}=L\left\{\left\{T_{1}\right\}\right\} \cdots\left\{\left\{T_{d-1}\right\}\right\}$. Let $M=L_{s}$ and $\mathcal{M}=L_{s}\left\{\left\{T_{1}\right\}\right\} \cdots\left\{\left\{T_{d-1}\right\}\right\}$. Then $Q_{s}=Q_{\mathcal{M}, s}, Q L_{s}=Q L_{\mathcal{M}, s}$ and

$$
P_{s}=P_{\mathcal{M}}=1 /\left(\pi_{1} D(M / K)\right) \mathcal{O}_{\mathcal{M}}=\left\{x \in \mathcal{M}: v_{\mathcal{M}}(x) \geq-v_{\mathcal{M}}\left(\pi_{1}\right)-v_{\mathcal{M}}(D(M / K))\right\},
$$

where $\pi_{1}$ is a uniformizer for $K_{1}=K\left(\kappa_{1}\right)$. Let $N_{s}=N_{\mathcal{M} / \mathcal{L}}$ and $\operatorname{Tr}_{s}=\operatorname{Tr}_{\mathcal{M} / \mathcal{L}}$. Let $\gamma_{s}$ denote a uniformizer of $\mathcal{M}$. For $t \geq s$ and $\mathcal{N}=L_{t}\left\{\left\{T_{1}\right\}\right\} \cdots\left\{\left\{T_{d-1}\right\}\right\}$ we define $N_{t / s}=N_{\mathcal{N} / \mathcal{M}}$ and $\operatorname{Tr}_{t / s}=\operatorname{Tr}_{\mathcal{N} / \mathcal{M}}$. Finally, let $\mathcal{L}_{s}^{\prime}$ be

$$
\bigcap_{t \geq s} N_{t / s}\left(L_{t}\left\{\left\{T_{1}\right\}\right\} \cdots\left\{\left\{T_{d-1}\right\}\right\}^{*}\right)
$$


Theorem 8.2.1. Let $r$ be maximal such that $L \supset K_{r}$ and $r^{\prime}$ minimal such that $L \cap K_{\pi} \subset K_{r^{\prime}}$. Let $s \geq \max \left\{r^{\prime}, n+r+\log _{q}\left(e\left(L / K_{r}\right)\right)\right\}$. Then $\operatorname{Tr}_{s}$ takes $\left(\pi^{s} / \pi_{1} \gamma_{s}\right) P_{s}$ to $\pi^{n} R_{\mathcal{L}}$ so that it induces a homomorphism

$$
\operatorname{Tr}_{s}: \frac{\frac{1}{\gamma_{s}} P_{s}}{\frac{\pi^{s}}{\pi_{1} \gamma_{s}} P_{s}} \longrightarrow \mathcal{L} / \pi^{n} R_{\mathcal{L}}
$$

and the following formula holds

$$
\left(\left\{N_{\mathcal{L}_{s} / \mathcal{L}}\left(a_{1}\right), a_{2}, \ldots, a_{d}\right\}, x\right)_{\mathcal{L}, n}=\left[\mathbb{T}_{\mathcal{L} / K}\left(\operatorname{Tr}_{s}\left(Q L_{s}\left(\left\{a_{1}, \ldots, a_{d}\right\}\right) l_{F}(x)\right)\right]_{f}\left(e_{n}\right)\right.
$$

for all $a_{1} \in \mathcal{L}_{s}^{\prime}=\cap_{t \geq s} N_{t / s}\left(\mathcal{L}_{t}\right)$ and all $a_{2}, \ldots, a_{d} \in \mathcal{L}^{*}$

Proof. Let $M=L_{t}$ and $\mathcal{M}=M\left\{\left\{T_{1}\right\}\right\} \cdots\left\{\left\{T_{d-1}\right\}\right\}$. Let $v$ be the normalized valuation $v_{\mathcal{M}} / v_{\mathcal{M}}(p)$ and let $R_{t, 1}=R_{\mathcal{M}, 1}, P_{t}=P_{\mathcal{M}}$. Since $K_{1} / K$ is totally ramified and $\left[K_{1} / K\right]=q-1$ then $\pi_{1}^{q-1} \sim \gamma_{t}^{e(M)}$, so $\pi_{1}$ divides $\gamma_{t}^{e(M) /(p-1)}$ and we have

$$
\frac{1}{\gamma_{t}} P_{t}=\frac{1}{\gamma_{t} \pi_{1} D\left(L_{t} / K\right)} \mathcal{O}_{\mathcal{L}_{t}} \subset \frac{1}{\gamma_{t}^{e(M) /(p-1)+1} D\left(L_{t} / K\right)} \mathcal{O}_{\mathcal{L}_{t}}=R_{t, 1}
$$

If $k<t$, then $\pi_{1} \mid \pi^{t-k} \gamma_{t}$, which implies $\left(\pi^{k} / \gamma_{t}\right) \mid\left(\pi^{t} / \pi_{1}\right)$ and so

$$
\frac{\pi^{t}}{\pi_{1} \gamma_{t}} P_{t} \subset \frac{\pi^{k}}{\gamma_{t}} R_{t, 1}
$$

Consider $t, k$ and $m$ as in Theorem 7.3.1. In particular, since $t=2 k+\alpha+1$ then $k<t$ and therefore we can look at the factorization of $\left.Q_{t}:\left(\mathcal{O}_{\mathcal{M}}^{*}\right)^{d} \rightarrow P_{t} /\left(\pi^{t} / \pi_{1}\right)\right) P_{t}$ 
into $R_{t, 1} /\left(\pi^{k} / \gamma_{t}\right) R_{t, 1}$. This is a $d$-dimensional derivation such that

$$
Q_{t}\left(T_{1}, \ldots, T_{d-1}, e_{t}\right)=T_{1} \cdots T_{d-1} / \pi^{t} l^{\prime}\left(e_{t}\right)=-T_{1} \cdots T_{d-1} c_{\beta} / l^{\prime}\left(e_{t}\right),
$$

by 8.12), where $\beta=(k, t)$ and $c_{\beta}=c_{\mathcal{M}, \beta}$. But from Lemma 7.2.1 we know that all the derivations satisfying such condition must coincide when reduced to $R_{t, 1} /\left(\pi^{m} / \gamma_{t}\right) R_{t, 1}$. Therefore the reduction of $Q_{t}$ to $R_{t, 1} /\left(\pi^{m} / \gamma_{t}\right) R_{t, 1}$ coincides with the derivation $D_{M, m}^{i}$ defined in 7.18 from Theorem 7.3.1. This allows us to replace the logarithmic derivative $\psi_{\mathcal{M}, m}$ defined in 7.19 from Theorem 7.3.1 by the logarithmic derivative

$$
Q L_{t}: K_{d}(\mathcal{M}) \rightarrow \frac{\frac{1}{\gamma_{t}} P_{t}}{\frac{\pi^{t}}{\pi_{1} \gamma_{t}} P_{t}}
$$

defined in (8.5), factored $\left(\bmod \left(\pi^{m} / \pi \gamma_{t}\right) P_{t}\right)$. That is, for $c \in \mathcal{L}_{t}^{*}$ and $a_{2}, \ldots, a_{d} \in \mathcal{L}^{*}$ we have

$$
\operatorname{Tr}_{\mathcal{M} / \mathcal{L}}\left(\psi_{\mathcal{M}, m}^{i}\left(c, a_{2}, \ldots, a_{d}\right)\right)=\operatorname{Tr}_{t}\left(Q L_{t}\left(c, a_{2}, \ldots, a_{d}\right)\right)
$$

But we know by Proposition 8.0 .6 that

$$
Q L_{s}\left(N_{t / s}(c), a_{2}, \ldots, a_{d}\right)=\operatorname{Tr}_{t / s}\left(Q L_{t}\left(c, a_{2}, \ldots, a_{d}\right) \quad\left(\bmod \frac{\pi^{s}}{\pi_{1} \gamma_{s}} P_{s}\right)\right.
$$

Suppose for the moment that $\operatorname{Tr}_{s}\left(\left(\pi^{s} / \pi_{1} \gamma_{s}\right) P_{s}\right) \subset \pi^{n} R_{\mathcal{L}}$, then taking $\operatorname{Tr}_{s}$ we get

$$
\begin{aligned}
\operatorname{Tr}_{t}\left(Q L_{t}\left(c, a_{2}, \ldots, a_{d}\right)\right) & =\operatorname{Tr}_{s}\left(\operatorname{Tr}_{t / s}\left(Q L_{t}\left(c, a_{2}, \ldots, a_{d}\right)\right)\right) \\
& =\operatorname{Tr}_{s}\left(Q L_{s}\left(N_{t / s}(c), a_{2}, \ldots, a_{d}\right)\right) \quad\left(\bmod \pi^{n} R_{\mathcal{L}}\right)
\end{aligned}
$$


Therefore if $a_{1} \in \mathcal{L}_{s}^{\prime}$ there exist a $c \in \mathcal{L}_{t}^{*}$ such that $N_{t / s}(c)=a_{1}$ and thus, by 8.15 and Proposition 7.1.2, identity (8.17) follows. It remains to prove that $\operatorname{Tr}_{s}\left(\left(\pi^{s} / \pi_{1} \gamma_{s}\right) P_{s}\right) \subset$ $\pi^{n} R_{\mathcal{L}}$ for $s \geq n+r+\log _{q}\left(e\left(L / K_{r}\right)\right)$

Let $x \in F\left(\mu_{\mathcal{L}}\right)$. Then $f(x) \equiv x^{q}(\bmod \pi x)$ implies

$$
v(f(x)) \geq \min \left\{v\left(x^{q}\right), v(\pi x)\right\}
$$

Let

$$
s^{\prime} \geq \log _{q}\left(\frac{e(L / K)}{q-1}\right)+1=r+\log _{q}\left(e\left(L / K_{r}\right)\right) .
$$

Then $v\left(f^{\left(s^{\prime}-1\right)}(x)\right) \geq \min \left\{v\left(x^{q^{s^{\prime}}-1}\right), v(\pi x)\right\}$. But

$$
\begin{gathered}
v\left(x^{q^{s^{\prime}-1}}\right)=q^{s^{\prime}-1} v(x)=\frac{e(L / K)}{q-1} \frac{v_{\mathcal{L}}(x)}{e(L / K) \alpha} \geq \frac{1}{\alpha(q-1)}=\frac{v(\pi)}{q-1}, \\
v(\pi x)>v(\pi),
\end{gathered}
$$

so $v\left(f^{\left(s^{\prime}-1\right)}(x)\right) \geq v(\pi) /(q-1)$. Thus by equation 8.16 applied to $x=f^{\left(s^{\prime}-1\right)}(x)$ we have

$v\left(f^{\left(s^{\prime}\right)}(x)\right) \geq \min \left\{v\left(\left(f^{\left(s^{\prime}-1\right)}(x)\right)^{q}\right), v\left(\pi f^{\left(s^{\prime}-1\right)}(x)\right)\right\} \geq\left(1+\frac{1}{q-1}\right) v(\pi)>\frac{v(\pi)}{q-1}$

By Lemma 8.2.1 below, we have $v\left(l_{F}\left(f^{\left(s^{\prime}\right)}(x)\right)\right)=v\left(f^{\left(s^{\prime}\right)}(x)\right)$, and since $v(\pi) /(q-1)=$ $v\left(\pi_{1}\right)$, then

$$
v\left(\pi^{s^{\prime}} l_{F}(x)\right) \geq(1+1 /(q-1)) v(\pi)=v(\pi)+v\left(\pi_{1}\right) .
$$

This implies $\left(\pi^{s^{\prime}-1} / \pi_{1}\right) T_{\mathcal{L}} \subset \mathcal{O}_{\mathcal{L}}$, where $T_{\mathcal{L}}=l_{F}\left(F\left(\mu_{\mathcal{L}}\right)\right)$. Taking duals with respect 
to $\mathbb{T}_{\mathcal{L} / K}$ we get

$$
\frac{\pi^{s^{\prime}-1}}{\pi_{1}} \frac{1}{D(L / K)} \mathcal{O}_{\mathcal{L}} \subset R_{\mathcal{L}}
$$

Since $\operatorname{Tr}_{s}\left(P_{s}\right) \subset P_{\mathcal{L}}$, by equation (8.6), then $\operatorname{Tr}_{s}$ takes

$$
\frac{\pi^{s}}{\pi_{1} \gamma_{s}} P_{s} \subset \frac{\pi^{s}}{\pi_{1} \pi_{L}} P_{s}
$$

to

$$
\frac{\pi^{s}}{\pi_{1} \pi_{L}} P_{\mathcal{L}}=\frac{\pi^{s}}{\pi_{1}^{2} \pi_{L}} \frac{1}{D(L / K)} \mathcal{O}_{\mathcal{L}} \subset \frac{\pi^{s-s^{\prime}+1}}{\pi_{1} \pi_{L}} R_{\mathcal{L}}
$$

Noticing now that $q \neq 2$, since $p \neq 2$, implies that $\pi_{1} \pi_{L} \mid \pi$, because $\left.v_{L}\left(\pi / \pi_{1} \pi_{L}\right)\right)=$ $e\left(L / L_{1}\right)(q-2)-1$. Thus, if $s \geq n+s^{\prime}$ then $\operatorname{Tr}_{s}$ takes $\left(\pi^{s} / \pi_{1} \gamma_{s}\right) P_{s}$ to $\pi^{n} R_{\mathcal{L}}$ and we conclude the theorem.

Lemma 8.2.1. For every element $w \in F\left(\mu_{\mathcal{L}}\right)$ such that

$$
v(w)>v(\pi) /(q-1)
$$

we have that

$$
v\left(l_{F}(w)\right)=v(w)
$$

Proof. The inequality $v(w)>v(\pi) /(q-1)$ is equivalent to $v\left(w^{q}\right)>v(\pi)+v(w)$, and since $f(x) \equiv x^{q}(\bmod \pi x)$ this implies $v(f(w))=v(\pi w)$. Thus $v\left(f^{(n)}(w)\right)=v\left(\pi^{n} w\right)$ and therefore we can take $n$ large enough such that $v\left(f^{(n)}(w)\right)>1 /(p-1)$. By Proposition 3.3 .1 we have that $v\left(l_{F}\left(f^{(n)}(w)\right)\right)=v\left(f^{(n)}(w)\right)$. Thus $v\left(\pi^{n} l_{F}(w)\right)=$ 
$v\left(\pi^{n} w\right)$, and this proves the lemma.

We restate the theorem in a simplified form.

Theorem 8.2.2. Let $r$ be minimal such that $L \cap K_{\pi} \subset K_{r}$ and $\mathcal{L}$ denote the field $L\left\{\left\{T_{1}\right\}\right\} \cdots\left\{\left\{T_{d-1}\right\}\right\}$. Let $s \geq n+r+\log _{q}\left(e\left(L / K_{r}\right)\right)$. Then

$$
\left(\left\{N_{\mathcal{L}_{s} / \mathcal{L}}\left(a_{1}\right), a_{2}, \ldots, a_{d}\right\}, x\right)_{\mathcal{L}, n}=\left[\mathbb{T}_{\mathcal{L} / K}\left(\operatorname{Tr}_{s}\left(Q L_{s}\left(\left\{a_{1}, \ldots, a_{d}\right\}\right) l_{F}(x)\right)\right]_{f}\left(e_{n}\right)\right.
$$

for all $a_{1} \in \mathcal{L}_{s}^{\prime}=\cap_{t \geq s} N_{t / s}\left(\mathcal{L}_{t}\right)$ and all $a_{2}, \ldots, a_{d} \in \mathcal{L}^{*}$. 


\section{Bibliography}

[1] Artin, E., and Hasse, H., Die beiden Ergänzungssätze zum Reziprozitätsgesetz der $l^{n}$-ten Potenzreste im Körper der $l^{n}$-ten Einheitswurzeln. Abh. Math. Sem. Univ. Hamburg 6 (1928), 146-162.

[2] J.W.S. Cassels and A. Frolich Algebraic Number Theory. Proc. Instructional Conf. (Brighton, 1965), $2^{\text {nd }}$ Edition London Mathematical Society 2010.

[3] J. Coates and A. Wiles, On the Birch and Swinnerton-Dyer conjecture. Invent. Math. 39 (1977), 223-251.

[4] I. B. Fesenko, Abelian extensions of complete discrete valuation fields, Séminarie de Théorie des Nombres Paris (1933-1934). LMS 235, 47-75.

[5] I. B. Fesenko, Sequential topologies and quotients of Milnor K-groups of higher local fields, (Russian) Algebra i Analiz 13 (2001), no. 3, 198221; translation in St. Petersburg Math. J. 13 (2002), no. 3, 485501.

[6] I.B. Fesenko, and S. V. Vostokov, Local fields and their extensions, second ed.,vol. 121 of Translations of Mathematical Monographs. AMS, Providence, RI, 2002.

[7] I. Fesenko and M. Kurihara, Invitation to Higher Local Fields. Geometry and Topology Monographs, 2000.

[8] P. Gille, and Szamuely, T. Central simple algebras and Galois cohomology, vol. 101 of Cambridge Studies in Advanced Mathematics. Cambridge University Press, Cambridge, 2006.

[9] K. Iwasawa, On explicit formulas for the norm residue symbol. J. Math. Soc. Japan 20 (1968), 151-164.

[10] K. Iwasawa, On some modules in the theory of cyclotomic fields. J. Math. Soc. Japan 16 (1963), 151-164. 
[11] K. Kato, A generalization of local class field theory by using K-groups II, Proc. Japan Acad. Ser. A Math. Sci. Volume 54, Number 8 (1978), 250-255.

[12] K. Kato, Generalized explicit reciprocity laws. Adv. Stud. Contemp. Math. (Pusan) 1 (1999), 57-126.

[13] K. Kato, p-adic Hodge theory and special values of zeta functions of elliptic cusp forms. Astérisque 295(2004), ix, 117-290, Cohomologies p-adiques et applications arithmétiques. III. MR 2104361

[14] V. Kolyvagin, Formal Groups and the Norm Residue Symbol, Math. USSR Izv. 15 (1980), 289-348.

[15] Kummer, E. Über die allgemeinen Reziprozitätsgesetze der Potenzreste. J. Reine Angew. Math.56 (1858), 270-279.

[16] M. Kurihara, The exponential homomorphism for the Milnor K-groups and an explicit reciprocity law, L. Reine Angew., 498 (1998), 201-221.

[17] M. Kurihara, Two types of complete discrete valuation fields., Geometry \& Topology Monographs, Volume 3, Part I, section 12, pages 109-112.

[18] S. Lang, Algebra. $2^{\text {nd }}$ Edition. Springer 2005

[19] J. Milnor, Algebraic K-theory and quadratic forms. Invent. Math. 9 (1970), 318344.

[20] M. Morrow, An introduction to higher dimensional local fields and adeles. 2012.

[21] J. Neukirch, Algebraic Number Theory. Springer 1991.

[22] A. N. Parshin Local class field theory. Trudy Mat. Inst. Steklov. 165 (1984), 143-170; English transl. in Proc. Steklov Inst. Math. 1985, no. 4(165).

[23] W. R. Scott, Group theory. Dover 1987.

[24] J. Silverman, Advanced topics in the arithmetic of elliptic curves. $2^{\text {nd }}$ Edition. Springer. 2009

[25] S. V. Vostokov, Explicit construction of class field theory for a multidimensional local field. Izv. Akad. Nauk SSSR Ser. Mat. (1985) no.2; English translation in Math. USSR Izv. 26(1986), 263288. 
[26] S. V. Vostokov, Hilbert pairing on a multidimensional complete field. Trudy Mat. Inst. Steklova, (1995); English translation in Proc. Steklov Inst. of Math. 208(1995), 7283

[27] Wiles, A. Higher explicit reciprocity laws. Ann. of Math. 107 (1978), no. 2, 235-254.

[28] A. N. Zinoviev, Generalized Artin-Hasse and Iwasawa formulas for the Hilbert symbol in a high-dimensional local field JMS, Vol 124, No. 1, 2004. 